\author{
Mon og r a p h \\ urn:1sid:zoobank.org:pub:A56D4F5D-FB1D-45C3-943A-EA64C90A3BAD
}

\title{
Acroterius gen. nov. (Araneae: Linyphiidae: Linyphiinae) with twelve new species from Yunnan, China
}

\author{
Muhammad IRFAN ${ }^{\circledR 1, *}$, Shahida BASHIR ${ }^{\circledR 2} \&$ Xianjin PENG ${ }^{\circledR 3, *}$ \\ ${ }^{1,3}$ College of Life Sciences, Hunan Normal University, Changsha, Hunan Province, \\ 410081, P.R. China. \\ ${ }^{1}$ Key Laboratory of Eco-environments in Three Gorges Reservoir Region (Ministry of Education), \\ School of Life Sciences, Southwest University, Chongqing 400715, P.R. China. \\ ${ }^{2}$ Department of Zoology, Government Postgraduate College, Jhang 35200, Punjab, Pakistan. \\ *Corresponding authors: irfanuos94@yahoo.com,xjpeng@126.com \\ 2Email:sbgcj@yahoo.com \\ ${ }^{1}$ urn:1sid:zoobank.org:author:4EE226DB-D810-4907-A4B6-B04F4E4241AB
${ }^{2}$ urn:1sid:zoobank.org:author:FACFE77D-08BE-4790-A853-9212BA61F4ED
${ }^{3}$ urn:1sid:zoobank.org:author:5CF67046-FAB4-43F1-AA72-ED2EB9741CD9
}

Abstract. Acroterius gen. nov. is described from Yuannan, together with twelve new species: A. absentus

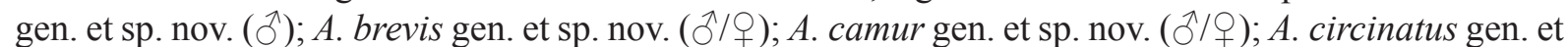

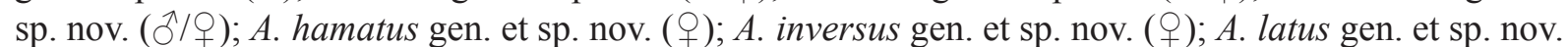

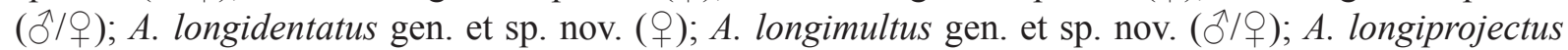
gen. et sp. nov. ( + ); A. ovatus gen. et sp. nov. ( + ), and A. parvus gen. et sp. nov. ( $(+)$. Detailed descriptions of genitalic characters and somatic features by means of photographs and line drawings, as well as comparisons with closely related species, are provided.

Keywords. Copulatory organ, Linyphiinae, Southeast Asia, sheet-web spiders, taxonomy.

Irfan M., Bashir S. \& Peng X. 2021. Acroterius gen. nov. (Araneae: Linyphiidae: Linyphiinae) with twelve new species from Yunnan, China. European Journal of Taxonomy 743: 1-53.

https://doi.org/10.5852/ejt.2021.743.1293

\section{Introduction}

Yunnan, the most southwestern province of China, is bordering with Laos, Vietnam, Myanmar and has seven climate zones (north tropical, south tropical, central subtropical, north subtropical, warm temperate, temperate and plateau climate areas). Its average temperature is about $5 \sim 24^{\circ} \mathrm{C}$. Yunnan Province has a very rich and diverse linyphiid spider fauna as was demonstrated by Irfan et al. (2019), who described a new genus Zhezhoulinyphia Irfan, Zhou \& Peng, 2019, and by Irfan \& Peng (2018, 2019a, 2019b), who mentioned three genera new to China along with six new species, i.e., Herbiphantes acutalis Irfan \& Peng, 2019, Houshenzinus tengchongensis Irfan \& Peng, 2018, Kaestneria valentissima Irfan \& Peng, 2018, Labullinyphia furcata Irfan \& Peng, 2019, Paikiniana operta Irfan \& Peng, 2018 and Parbatthorax proiectus Irfan \& Peng, 2019, from Yunnan. Zhao \& Li (2017) described Callosa 
Zhao \& Li, 2017, a new troglobitic genus from Yunnan. Eight new genera and 30 new species have been described in the survey of linyphiid spiders from Xishuangbanna, Yunnan (Zhao \& Li 2014). Most of the species described from Yunnan Province are not found in other parts of China and will probably be found in the Oriental region.

The fauna of Linyphiinae Blackwall, 1859 from China comprises 69 species belonging to 16 genera (Li \& Lin 2016). We here describe a new genus after having checked the available literature and based on morphological characteristics, Acroterius gen. nov., which closely resembles the genera Kaestneria Wiehle, 1956; Zhezhoulinyphia (also reported from Yunnan); Diplostyla Emerton, 1882; Laetesia Simon, 1908 and Laperousea Dalmas, 1917, whereas no phylogenetic evidence of linyphiin spiders from southeast Asia was discovered. More systematic and phylogenetic studies need to be done to confirm the relationship between the closely related genera. Spider collections during 2002-2006 from Yunnan have revealed the presence of Acroterius gen. nov. with twelve new species, all described here, with locality maps also provided (Figs 38-39).

\section{Material and methods}

Specimens were collected by hand picking and beating shrubs and were kept in $75 \%$ ethanol. After dissection, epigyna were cleared in trypsin enzyme solution before examination and photography. The left male palps were used for description and illustration. Specimens were examined and measured with a Leica M205C stereo microscope. Photos were taken with a digital camera Canon PowerShot G12 mounted on an Olympus BX53 and Leica MC170 HD mounted on a Leica M205C and were stacked by Helicon Focus software (3.10. free). Maps were created using ArcMap 10.2, and then modified using Adobe Photoshop CS2 Extended (Figs 38-39). Leg measurements are given in the following order: total length (femur, patella + tibia, metatarsus, tarsus). All measurements are given in millimeters (mm). The terminology used in text and figure legends follows Irfan et al. (2019). Specimens are deposited in the collection of the College of Life Sciences, Hunan Normal University (HNU).

\section{Abbreviations}

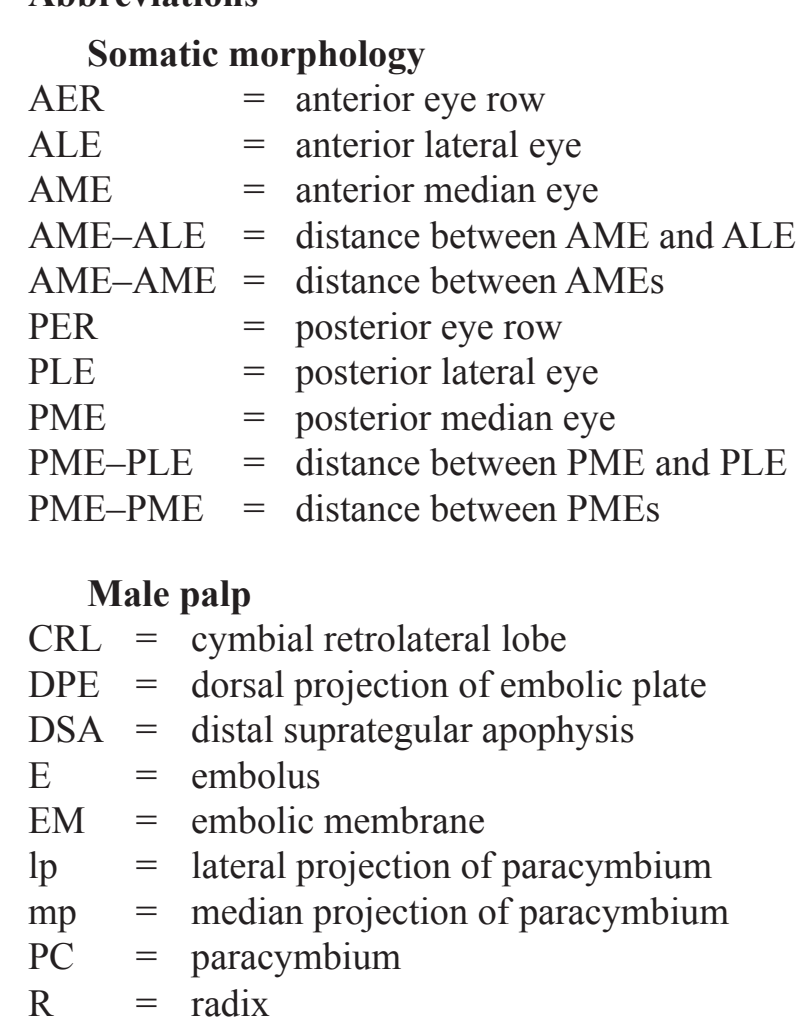



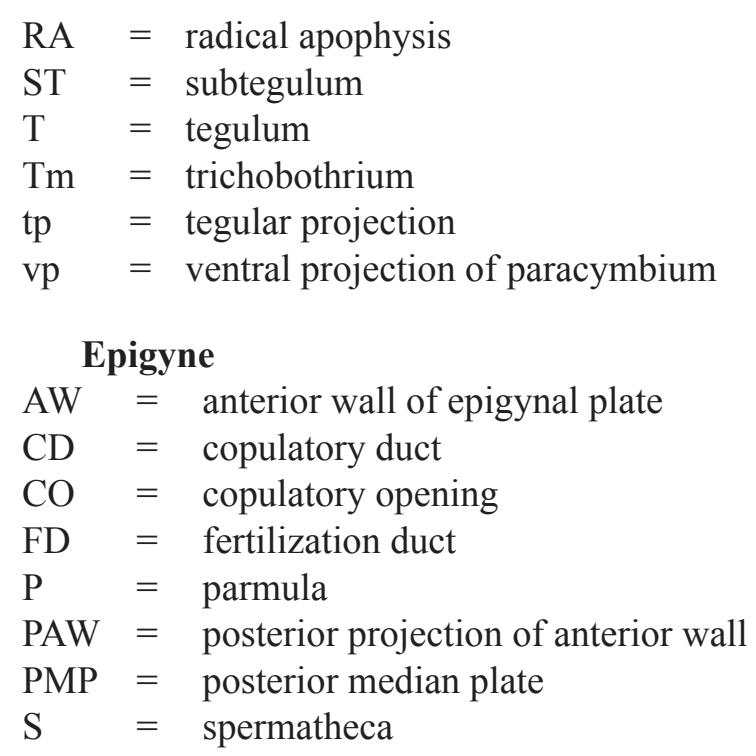

\title{
Results
}

\author{
Class Arachnida Cuvier, 1812 \\ Order Araneae Clerck, 1757 \\ Family Linyphiidae Blackwall, 1859 \\ Subfamily Linyphiinae Blackwall, 1859 \\ Acroterius gen. nov. \\ urn:1sid:zoobank.org:act:EEA7B37A-300B-43D9-87F3-82A380BC686D
}

\section{Type species}

Acroterius brevis gen. et sp. nov.

\section{Diagnosis}

Acroterius gen. nov. resembles Diplostyla; Kaestneria Wiehle, 1956; Laetesia Simon, 1908; Laperousea Dalmas, 1917 and Zhezhoulinyphia in: epigyne with parmula, originates from the posterior margin of posterior median plate with a socket ventrally (Fig. 8A-C; Ivie 1969: figs 105-108; van Helsdingen 1972: fig. 8; Millidge 1988: fig. 173; Irfan \& Peng 2018: figs 5c-d, 6c-d; Irfan et al. 2019: figs 1-2, 7, 9-10). In the male palp, the distal margin of the radix is semicircular and has teeth as in Laetesia, Laperousea and Zhezhoulinyphia (Figs 6A-C, 7A-B; Millidge 1988: figs 145-146; Irfan et al. 2019: figs $4 \mathrm{a}-\mathrm{b}, \mathrm{d}, 5 \mathrm{c}-\mathrm{e}, 6 \mathrm{a}-\mathrm{b})$; the embolus and embolic membrane arise from the dorsal side of the distal margin of the radix as in Zhezhoulinyphia (Figs 6A-C, 7A-B; Irfan et al. 2019: figs 4a-b, d, 5c-e, 6a-b). It can be distinguished by the anterior wall of the epigyne which has a posterior projection on each side (Fig. 8A-B), but in species of Laetesia, the posterior arm of the anterior wall of the epigynal plate is long, with a copulatory opening on the posterior ventral end (Millidge 1988: figs 173, 187), and the posterior projection of the anterior wall absent in species of Diplostyla, Kaestneria and Zhezhoulinyphia (van Helsdingen 1972: fig. 8; Millidge 1988: fig. 173; Irfan \& Peng 2018: figs 5c-d, 6c-d; Irfan et al. 2019 : figs 1-2, 7, 9-10). The spermathecae are situated near the base of the parmula (Fig. 8A-B) in Acroterius gen. nov., but towards the periphery of epigyne in species of Kaestneria, Laetesia and Zhezhoulinyphia (Millidge 1988: figs 187-189; Tao et al. 1995: figs 80-81; Irfan et al. 2019: figs 1-2, 7, 9-10). The cymbium has a cymbial retrolateral lobe (Figs 6B-D, 7B) as in species of Zhezhoulinyphia (Irfan et al. 2019: figs 4b, 5b, g, 6b), but it is absent in Kaestneria and Laetesia (Millidge 1988: figs 143-144; Paquin \& Dupérré 2003: figs 1544, 1547). The distal arm of the paracymbium of Acroterius gen. nov. has two or three projections and covers most of the proximal part of paracymbium (Figs 6B-C, 7B), the 
distal part of the paracymbium of all species of Kaestneria and Laetesia is simple U- or V-shaped and doesn't cover the proximal part (Millidge 1988: figs 187-189; Paquin \& Dupérré 2003: figs 1544, 1547) and there is a large, distal arm longer than wide, tip pendulum-shaped in species of Zhezhoulinyphia (Irfan et al. 2019: figs 4b, 5f, 6b). The distal end of the distal suprategular apophysis of Acroterius gen. nov. and Kaestneria is not notched (Figs 6B-C, 7B; Tao et al. 1995: figs 77-78) (but notched in species of Laetesia, Millidge 1988: fig. 146), and proximally broad with teeth, has a distal part strongly curved into an inversed U-shape, almost touching the distal margin of the paracymbium in species of Zhezhoulinyphia (Irfan et al. 2019: figs 4b, 5b, g, 6b). The tegulum of Acroterius gen. nov. has a small tegular projection ventrally (Figs 6B-C, 7B), which is absent in Kaestneria (Paquin \& Dupérré 2003: figs 1544, 1547; Zhao \& Li 2014: figs 47b, 49b) and Laetesia (Millidge 1988: fig. 146). The embolic division of Acroterius gen. nov. comprises a distal semicircular part of the radix with a serrated margin anteriorly; the embolus and embolic membrane arise from the terminal part of the radix (Figs $6 \mathrm{~A}-\mathrm{C}$, 7A-B) as in species of Zhezhoulinyphia (Irfan et al. 2019: figs 4a-b, 5c-e, 6a-b); the embolus and median membrane arise from the lateral (inner) side of the plate in Kaestneria and Laetesia (Millidge 1988: figs 145, 149; Zhao \& Li 2014: figs 47b, 49b) whereas in Diplostyla the embolus arises near the base of the cymbium and extends parallel along the full length of cymbium (Ivie 1969: figs 107-108). In Acroterius gen. nov. the proximal end of the radix has a radical apophysis (Figs 6A-C, 7A-B) as in species of Zhezhoulinyphia (Irfan et al. 2019: figs 4a-b, 5c-e, 6a-b), but it is absent in Diplostyla, Kaestneria and Laetesia (Ivie 1969: figs 107-108; Millidge 1988: figs 145, 149; Zhao \& Li 2014: figs 47b, 49b).

\section{Etymology}

The generic name is an arbitrary combination of letters. Gender masculine.

\section{Description}

MEASUREMENTS. Large sized, male total length 2.66-3.61; female total length 2.66-4.15.

HEAD. Cephalic region slightly elevated, yellowish to dark brown; fovea, cervical and radial grooves distinct; sternum longer than wide, green to yellowish to dark brown with spine-like hairs; labium wider than long, dark brown; maxillae long, distal end broader with scopulae. Eye region narrow, AER recurved, PER procurved slightly wider.

LEGS. All legs with annuli; patella of each leg with two spines; chaetotaxy: 2-2-2-2; Tm I and Tm IV present.

AвDOMEn. Oval, dorsally with pattern extending down to the base of spinnerets followed by irregular white patches dorso-laterally, and ventrally pale, greenish to brown with or without irregular white patches (Fig. 4A-B).

Male palp (Figs 1, 3, 6, 7, 10-12, 15-16, 24, 26, 30-31). Patella shorter than tibia, dorsally with a long spine; tibia cone shaped, with two retrolateral trichobothria, with some short and long setae on all surfaces; paracymbium highly sclerotized, proximal part simple, somewhat rectangular, distal arm with two to three projections (characterized as lateral, median and ventral projections); cymbium with retrolateral lobe, covered with long thick spines; tegulum with small projection ventrally; distal suprategular apophysis long, basally covered by embolic division. Radix with distal broad semicircular serrated part giving rise to embolus and embolic membrane, at proximal end with small radical apophysis and dorsal projection of embolic plate; dorsal projection of embolic plate long, slightly overlapping cymbium; embolic membrane curved and approachnig distal semicircular part, in prolateral view; embolus stout, short with pointed end.

Epigyne (Figs 8, 13, 17, 21A-D, 22, 27, 29A-D, 32, 34B-E, 36A-D, 37). Anterior wall of epigyne wider than long; posterior margin of anterior wall with a projection on each side; copulatory openings 
situated inside the atrium between the anterior wall and posterior median plate, connected with long, semicircular copulatory ducts joined to the spermathecae. Posterior median plate posteriorly with a parmula of variable length with a socket ventrally. Spermathecae slender, present near the posterior median plate. Fertilization ducts long, present laterally on the posterior median plate, extending mesally.

\section{Distribution}

China (Yunnan Province, Figs 38-39).

Acroterius absentus gen. et sp. nov. urn:1sid:zoobank.org:act:4E49A779-9374-40FB-B842-BEECDAD450A4

Figs $1-4,38$

\section{Differential diagnosis}

The new species can be distinguished from all other species of Acroterius gen. nov. by the absence of radical apophysis (Figs 1A-C, 3A-B).

\section{Etymology}

The species name comes from the Latin adjective 'absens', meaning 'absent', referring to the radical apophysis absent in male palp.

\section{Type material}

\section{Holotype}

CHINA - गे; Yunnan Province, Baoshan City, Nankang Yakou (National 320 Road); $24.43717^{\circ}$ N, 98.46054 E; alt. 2186 m, 27 Oct. 2003; Guo Tang leg.; HNU-Tang031027.

\section{Description}

\section{Male (holotype)}

MEASUREMENTS AND COLOUR. Total length: 3.61. Carapace 1.34 long, 1.14 wide, cephalothorax yellowish to light brown; clypeus 0.56 high (Fig. 4A-B); abdomen 2.27 long, 0.89 wide. Eye sizes and interdistances: AME 0.09, ALE 0.12, PME 0.09, PLE 0.11, AME-AME 0.05, PME-PME 0.08, AME-ALE, 0.12, PME-PLE 0.11, ALE-ALE 0.54, PLE-PLE 0.61, ALE-PLE contiguous.

Chelicerae. With five promarginal and five retromarginal teeth, promargin with deep depression and with small teeth except proximal two long; proximal second tooth much longer and almost touching upper margin of fang, distal three teeth small (Fig. 2A-B).

LENGTH OF LEGS. I $=7.48(1.89,2.48,2.03,1.08), \mathrm{II}=5.9(1.83,1.92,1.32,0.83), \mathrm{III}=4.11(1.41,1.16$, $0.97,0.57), \mathrm{IV}=5.52(1.72,1.69,1.49,0.62)$. Leg formula I-II-IV-III.

PALP (Figs 1A-D, 3A-B). Paracymbium highly sclerotized, distal part with three projections (Figs 1B, 3B), lateral projection small, triangular; median projection broad, tongue-shaped and ventral projection thumb-like (Figs 1B, 3B); the distal suprategular apophysis robust with pointed end (Figs 1A-C, 3A-B); radical apophysis absent (Figs $1 \mathrm{~A}-\mathrm{C}, 3 \mathrm{~A}-\mathrm{B}$ ). Embolus originating from distal serrated part of radix, stout, short with pointed end (Figs $1 \mathrm{~A}-\mathrm{C}, 3 \mathrm{~A}-\mathrm{B}$ ).

\section{Female}

Unknown.

\section{Distribution}

China (Yunnan Province, Fig. 38). 


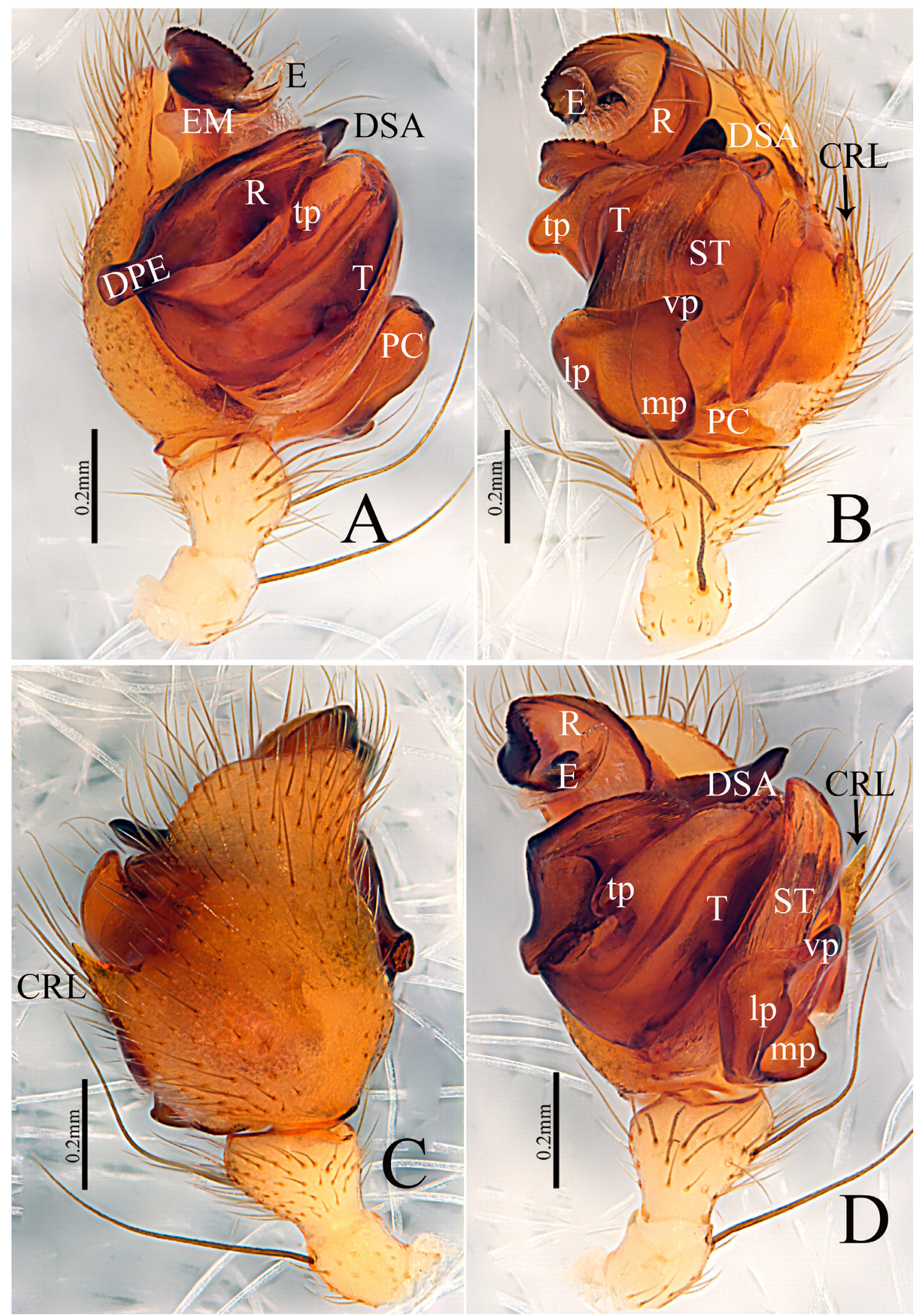

Fig. 1. Acroterius absentus gen. et sp. nov., ô, holotype (HNU-Tang031027), palp. A. Prolateral view. B. Retrolateral view. C. Dorsal view. D. Ventral view. 


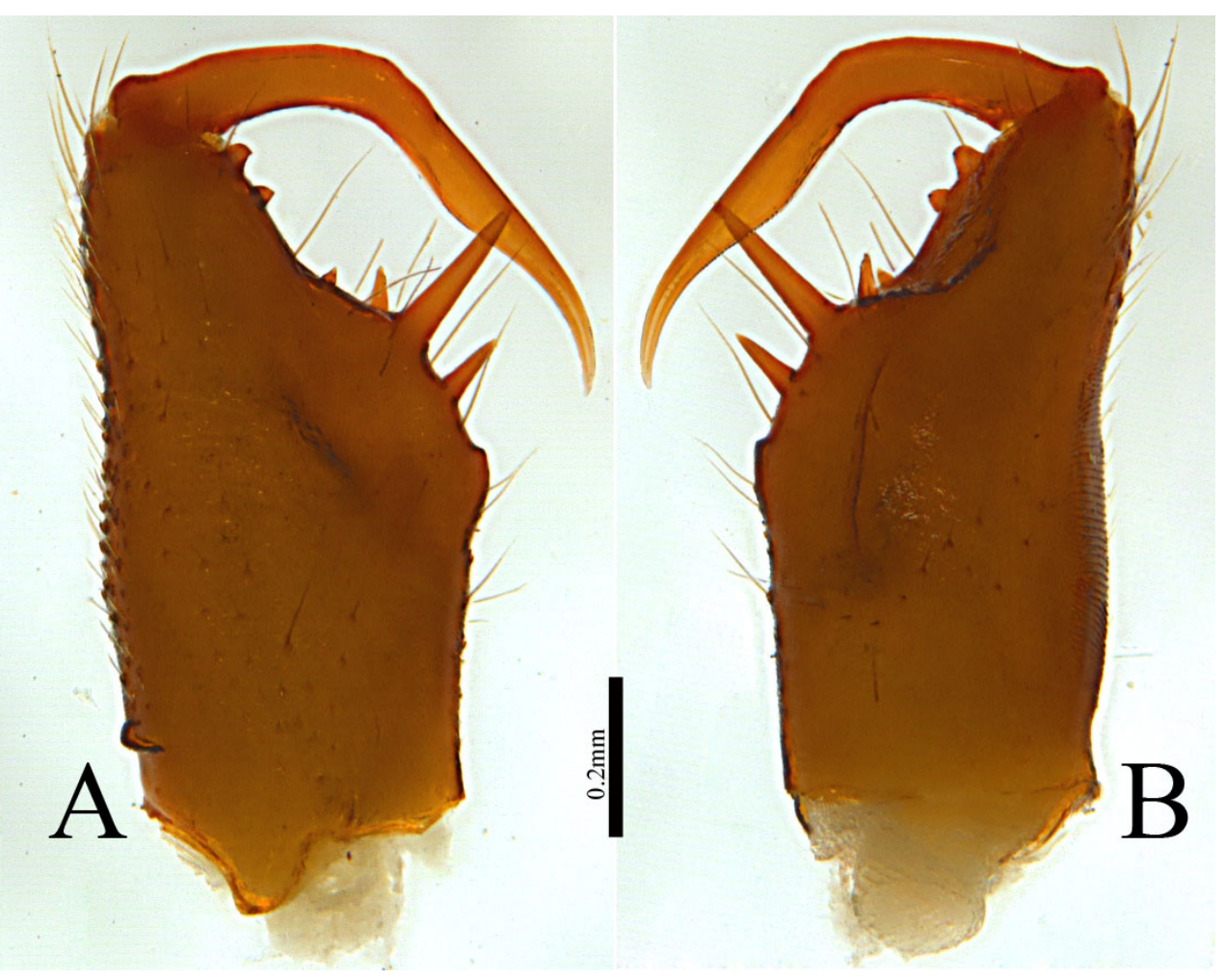

Fig. 2. Acroterius absentus gen. et sp. nov., §̊, holotype (HNU-Tang031027). A. Chelicera, dorsal view. B. Chelicera, ventral view.
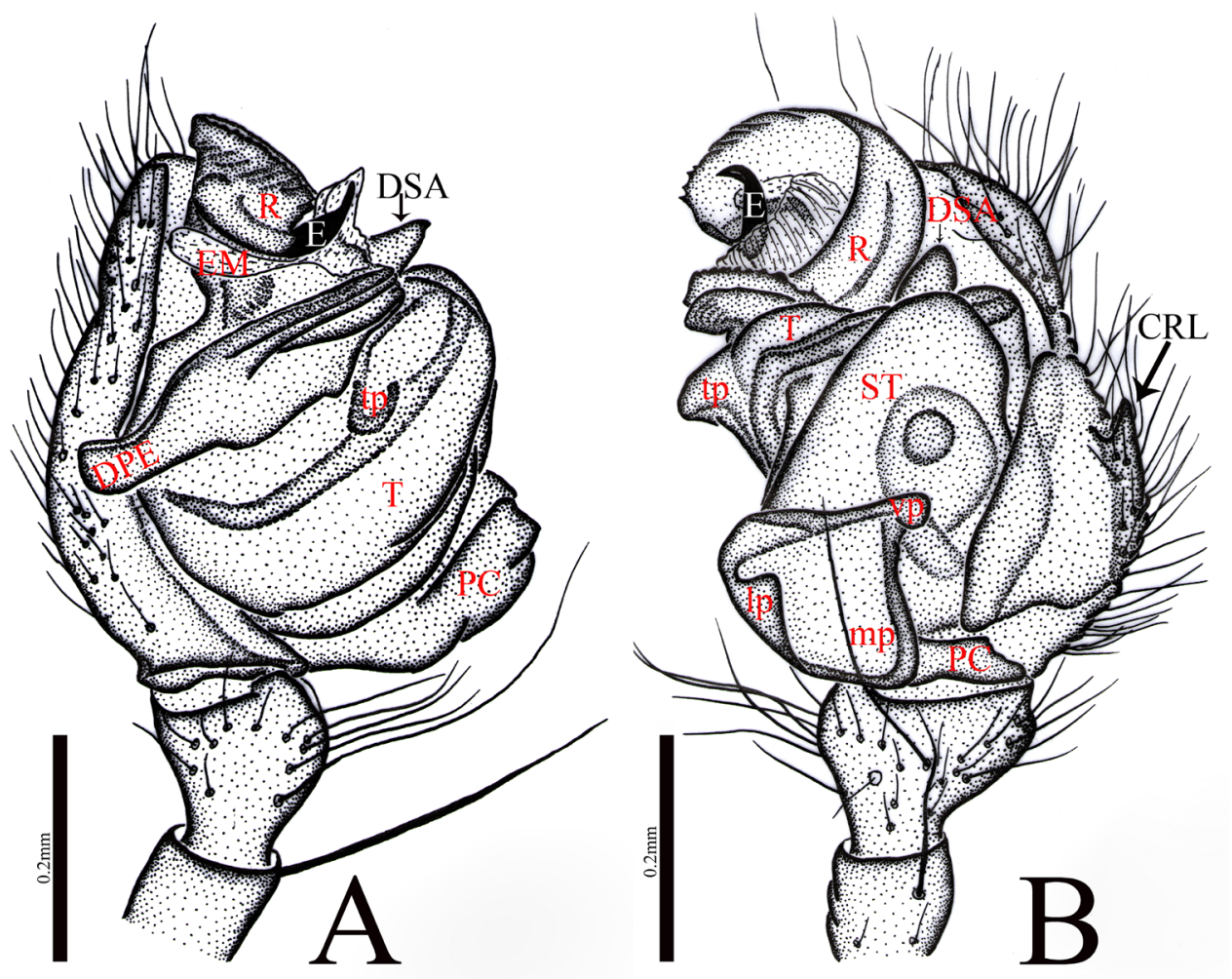

Fig. 3. Acroterius absentus gen. et sp. nov., ô, holotype (HNU-Tang031027), palp. A. Prolateral view. B. Retrolateral view. 


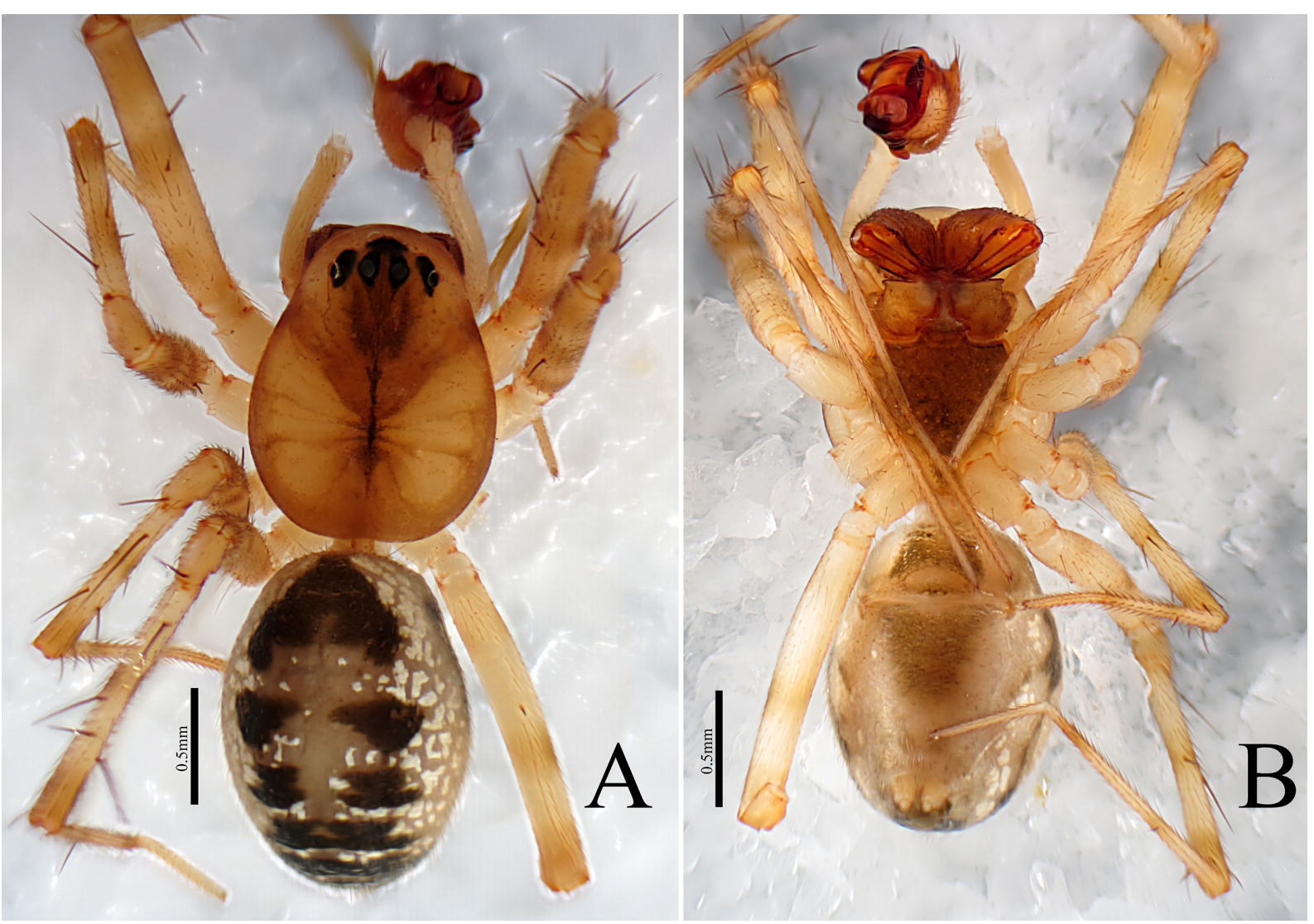

Fig. 4. Acroterius absentus gen. et sp. nov., $\widehat{\jmath}$, holotype (HNU-Tang031027). A. Habitus, dorsal view. B. Habitus, ventral view.

Acroterius brevis gen. et sp. nov. urn:1sid:zoobank.org:act:F041D21F-15E7-4C22-895A-4AF2565105D4

Figs 5-9, 38

\section{Differential diagnosis}

The new species resembles $A$. camur gen. et sp. nov. in distal arm of paracymbium with three projection (Figs 6B, 7B, 10B, 11B), but can be distinguished from A. camur gen. et sp. nov. and all other Acroterius gen. nov. species by the ventral view, radical apophysis triangular and reaches to base of embolus (Figs 6D, 7A), but thumb-shaped and extends above embolus in A. camur gen. et sp. nov. (Figs 10D 12A). Spermathecae L-shaped, present near posterior median plate (Fig. 8A-B), but hook-shaped and separated by one and half-length of spermathecae in $A$. camur gen. et sp. nov. (Fig. 13A-B). Parmula 0.47 long (Fig. 8D), but 0.21 long in A. camur gen. et sp. nov. (Fig. 13D).

\section{Etymology}

The species name comes from the Latin adjective 'brevis' meaning 'short', referring to the short radical apophysis in male palp.

\section{Type material}

\section{Holotype}

CHINA - ग; Yunnan Province, Fugong County, Lumadeng Township, Yaping Village, Shibali; 27.16515 N, 98.77975 E; alt. 2527 m; 1 May 2004; Guang-xu Peng leg.; HNU-20040501-1. 
Paratypes $(2 q+)$

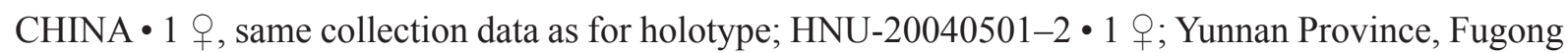
County, Yaping, $3 \mathrm{~km}$ to Shibali; 27.1775 N, 98.7550 E; alt. $2527 \mathrm{~m}$; 4 May 2004; Heng-mei Yan and Guang-xu Peng leg.; HNU-20040504.

\section{Description}

\section{Male (holotype)}

Measurements And COLOuR. Total length: 2.66. Carapace 1.40 long, 1.14 wide, cephalothorax dark brown; clypeus 0.56 high (Fig. 9A-B); abdomen 2.03 long, 1.60 wide. Eye sizes and interdistances: AME 0.08, ALE 0.09, PME 0.08, PLE 0.07, AME-AME 0.03, PME-PME 0.06, AME-ALE 0.08, PME-PLE 0.07, ALE-ALE 0.45, PLE-PLE 0.49.

Chelicerae. With four promarginal and four retromarginal teeth; proximal teeth of reteromargin much longer than distal one.

LENGTH OF LEGS. I $=4.89(1.63,1.47,1.16,0.63), \mathrm{II}=3.74(1.11,1.31,0.83,0.49), \mathrm{III}=3.17(0.88,0.96$, $0.82,0.51), \mathrm{IV}=4.35(1.26,1.26,1.17,0.66)$. Leg formula I-IV-II-III.

PaLp (Figs 6A-D, 7A-B). Paracymbium highly sclerotized, distal arm with three projections; lateral projection large, curved, beak-shaped; median projection tongue-shaped; ventral projection sclerotized, thumb-shaped with blunt end, overlapping subtegulum (Figs 6B, 7B); distal suprategular apophysis long, robust with distal pointed end (in ventral view) (Figs 6B-D, 7B). Radix with small radical apophysis

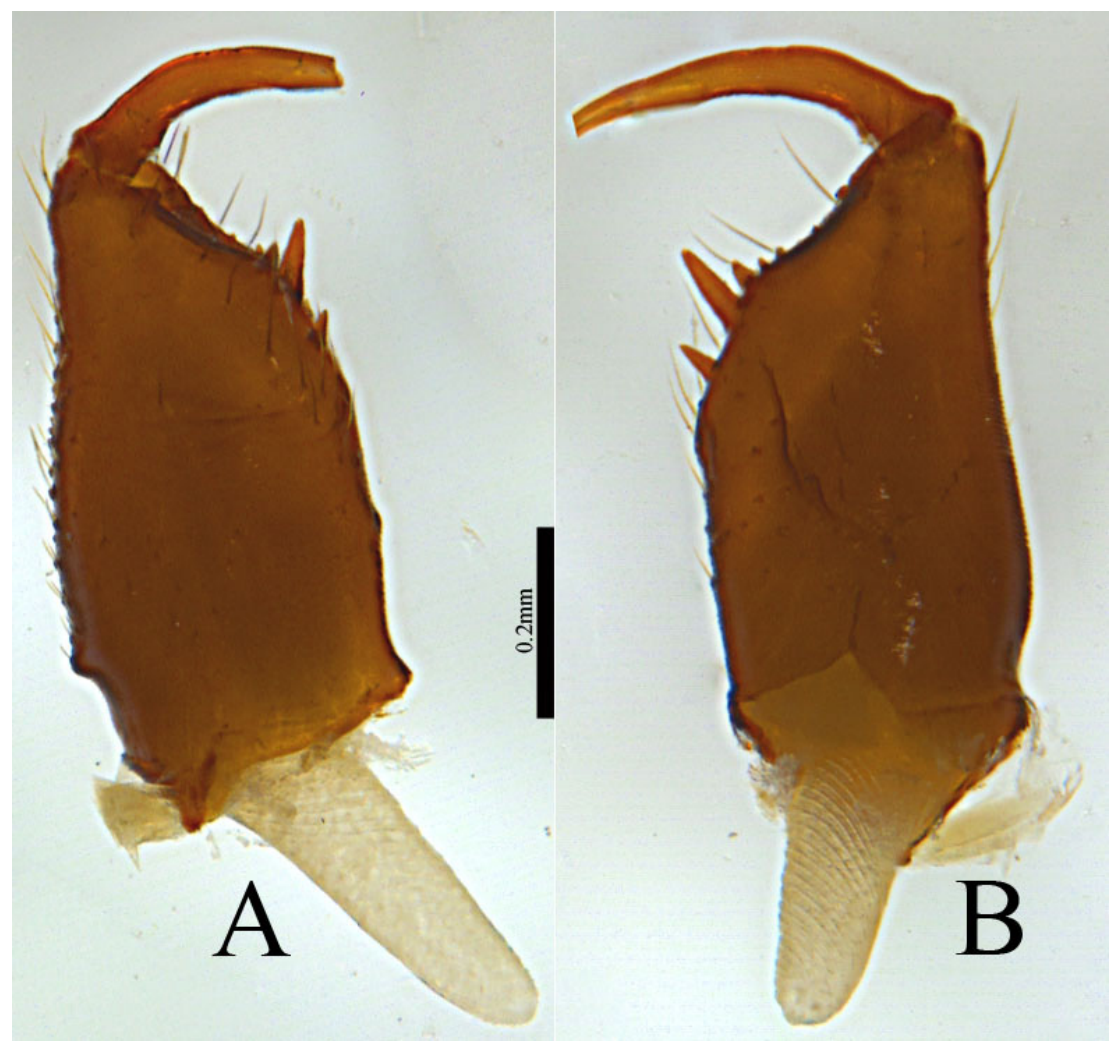

Fig. 5. Acroterius brevis gen. et sp. nov., §̂, holotype (HNU-20040501-1). A. Chelicera, dorsal view. B. Chelicera, ventral view. 

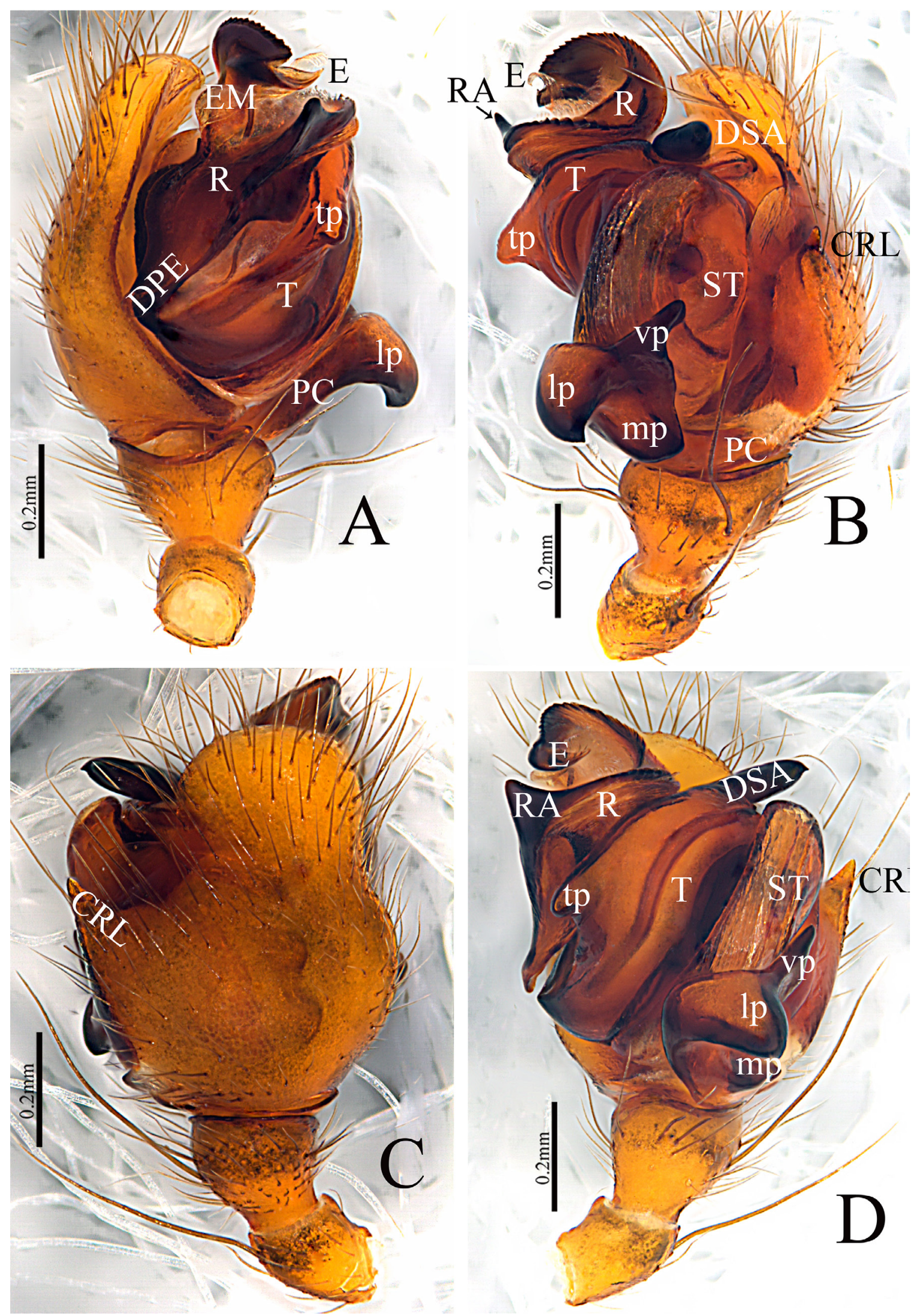

Fig. 6. Acroterius brevis gen. et sp. nov., $\hat{\jmath}$, holotype (HNU-20040501-1), palp. A. Prolateral view. B. Retrolateral view. C. Dorsal view. D. Ventral view. 
with pointed end (Figs 6A-D, 7A-B); radical apophysis somewhat triangular with blunt end (Figs 6A, 7A). Embolus stout, short with pointed end (Figs 6A-D, 7A-B).

Female (HNU-20040501-2, Fig. 9C-D)

MEASUREMENTS AND COLOUR. Total length: 2.97. Carapace 0.94 long, 0.83 wide, cephalothorax dark brown; fovea, cervical and radial grooves distinct; clypeus 0.27 high (Fig. 9C-D); abdomen 1.8 long, 1.05 wide. Eye sizes and interdistances: AME 0.06, ALE 0.09, PME 0.08, PLE 0.07, AME-AME 0.06, PME-PME 0.07, AME-ALE 0.07, PME-PLE 0.07, ALE-ALE 0.41, PLE-PLE 0.45, ALE-PLE contiguous.

LENGTH OF LEGS. I $=4.40(1.23,1.39,1.03,0.75), \mathrm{II}=3.92(1.15,1.28,0.91,0.58), \mathrm{III}=3.07(0.94,0.93$, $0.71,0.49), \mathrm{IV}=4.11(1.13,1.35,0.96,0.67)$. Leg formula I-IV-II-III. Tm I and Tm IV present. Patella of each leg with two spines. Tibial dorsal spine formula: 2-2-2-2.

Epigyne. Parmula 0.47 long, 0.41 wide at base (Fig. 8A-C); the posterior margin of the anterior wall of epigynal plate with a beak-shaped projection on each side (Fig. 8A-C). Spermathecae slender, L-shaped (Fig. 8D).
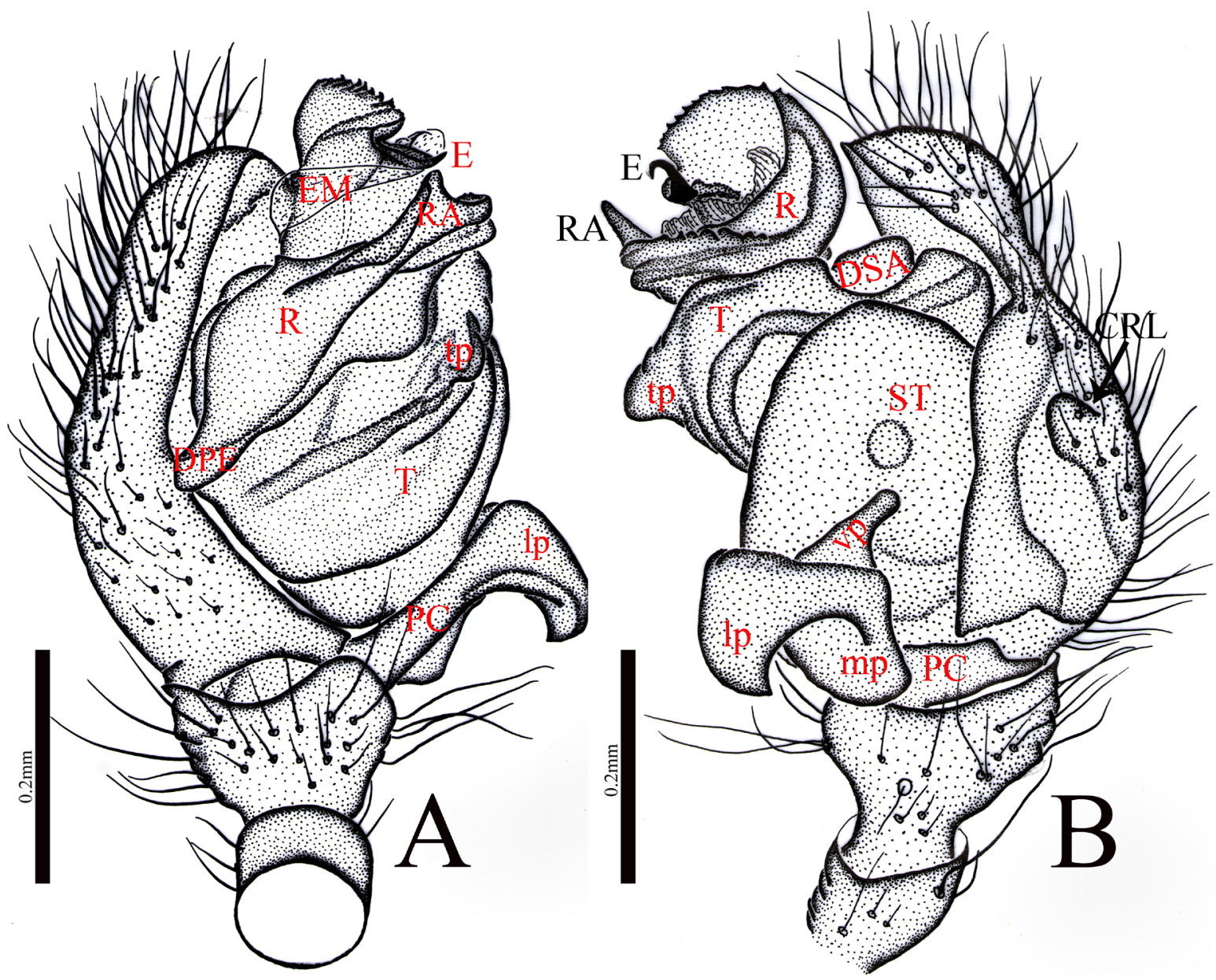

Fig. 7. Acroterius brevis gen. et sp. nov., ô, holotype (HNU-20040501-1), palp. A. Prolateral view. B. Retrolateral view. 

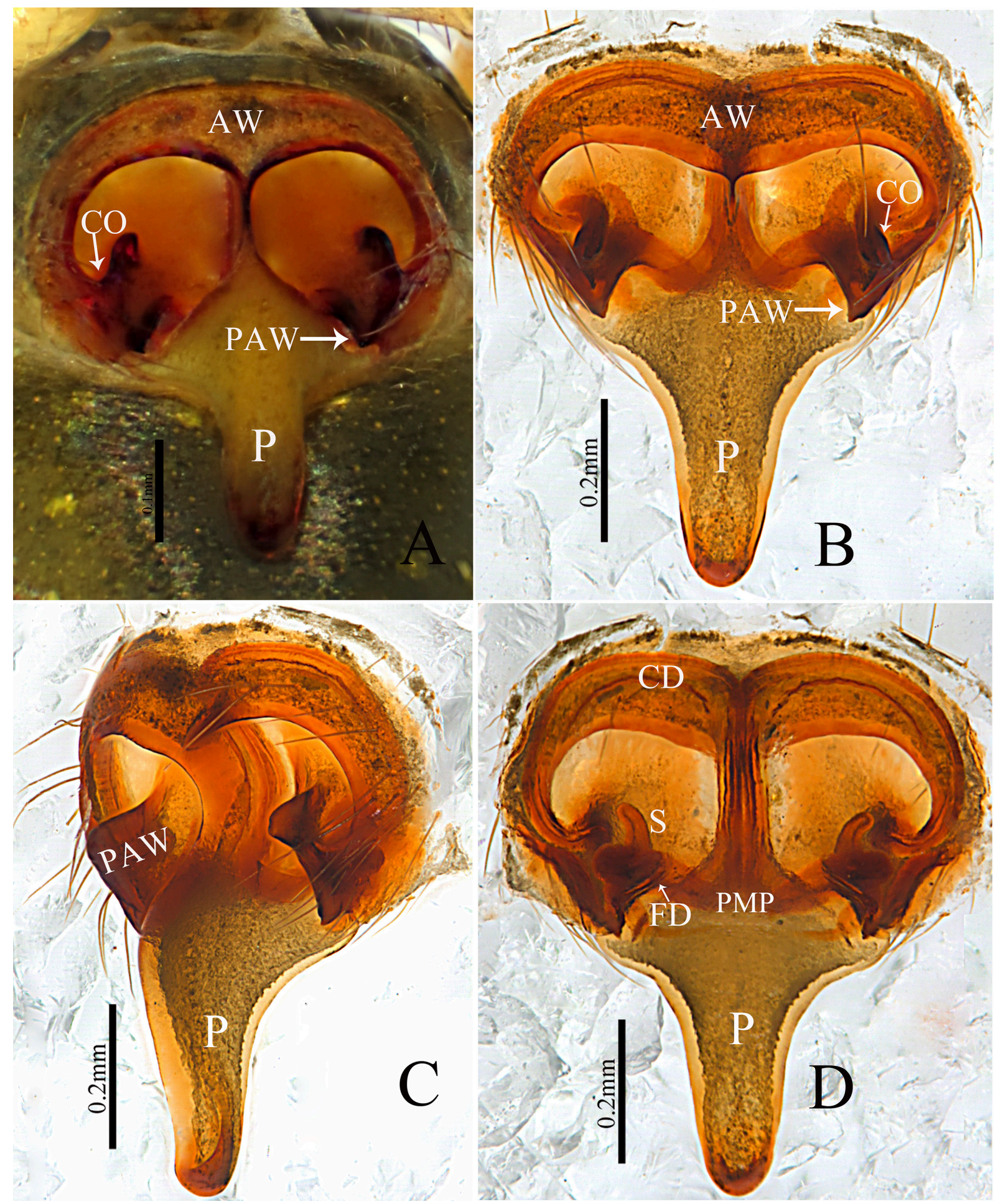

Fig. 8. Acroterius brevis gen. et sp. nov., + , paratype (HNU-20040501-2). A-B. Epigyne, ventral view. C. Epigyne, lateral view. D. Vulva, dorsal view. 

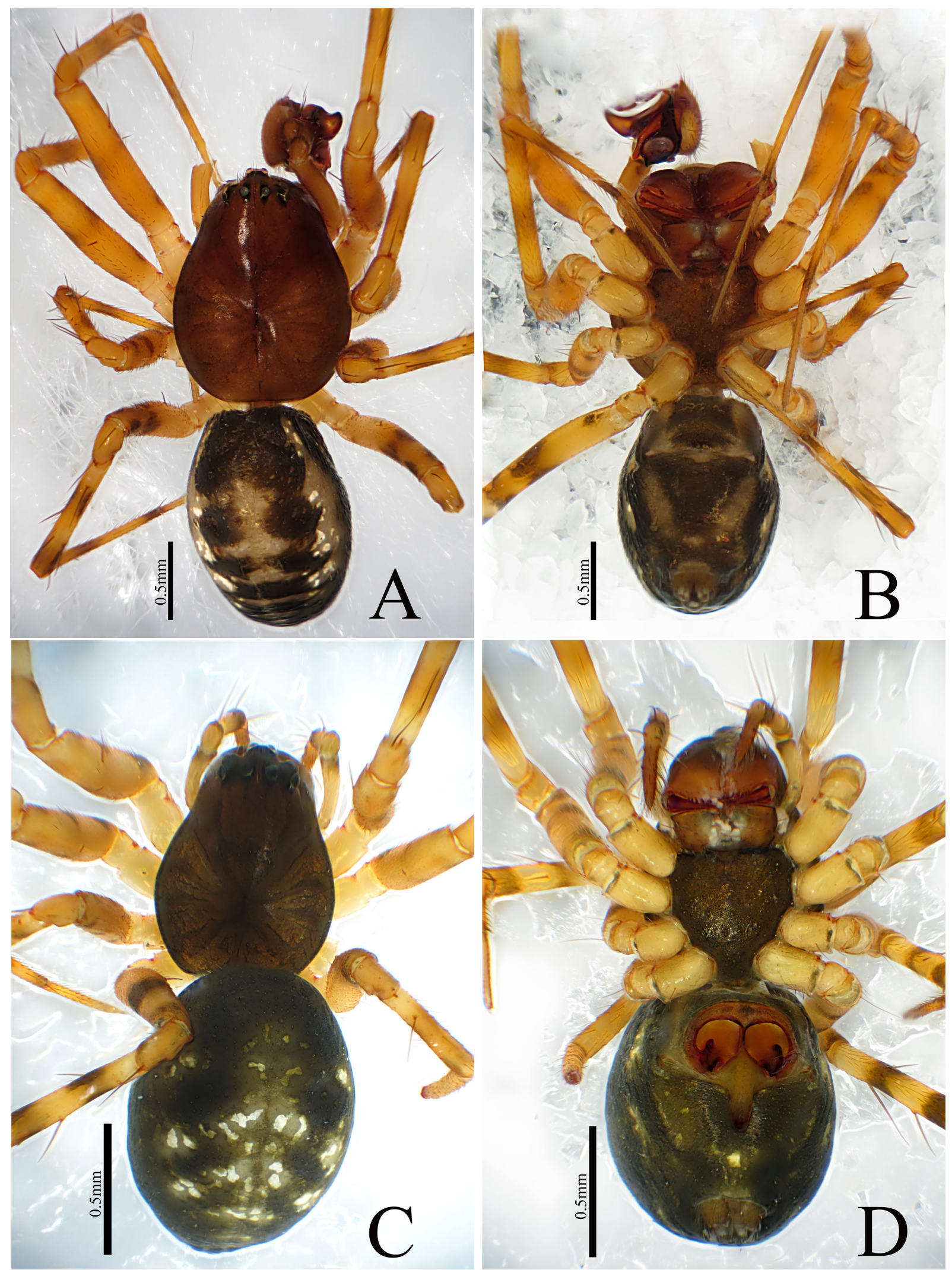

Fig. 9. Acroterius brevis gen. et sp. nov. A-B. $\widehat{\jmath}$, holotype (HNU-20040501-1). C-D. + , paratype (HNU-20040501-2). A, C. Habitus, dorsal view. B, D. Habitus, ventral view. 


\section{Distribution}

China (Yunnan Province, Fig. 38).

Acroterius camur gen. et sp. nov. urn:1sid:zoobank.org:act:B86B9669-F527-44A7-BAF0-40B777161195

Figs 10-14, 38

\section{Differential diagnosis}

The new species resembles Acroterius brevis gen. et sp. nov. (Figs 6A-D, 7A-B) in having similar embolus in the male palp and a short parmula in the epigyne (Figs 10A-D, 11A-D, 12A-B) as in $A$. inversus gen. et sp. nov. and A. longidentatus gen. et sp. nov.; it can be distinguished by the distal arm of paracymbium with three projections and similar embolus, the radical thumb-shaped apophysis extends above the embolus in ventral view (Figs 10D, 12A), but is triangular and reaches to the base of the embolus in A. brevis gen. et sp. nov. (Figs 6D, 7A). Spermathecae hook-shaped, separated by one and half-length of spermathecae (Fig. 13A-B), but L-shaped and present near the posterior median plate in A. brevis gen. et sp. nov. (Fig. 8A-D). Parmula somewhat triangular (Fig. 13A-D), but tongue-shaped in A. brevis gen. et sp. nov. (Fig. 8A-C), anteriorly rectangular and posteriorly spoon-shaped in A. inversus gen. et sp. nov. (Fig. 22A-C), finger-shaped in A. longidentatus gen. et sp. nov. (Fig. 29A-C).

\section{Etymology}

The species name comes from the Latin adjective 'camur', meaning 'curved' and referring to the curved distal suprategular apophysis in the male palp.

\section{Type material}

\section{Holotype}

CHINA -1 ; ; Yunnan Province, Gongshan County, Bingzhongluo Township, Niwaluo He, just below Nujiang Road; $28.02499^{\circ}$ N, $98.62564^{\circ}$ E; alt. 1610 m; 8 Oct. 2002; D.H. Kavanaugh, P.E. Marek and Hong-bin Liang leg.; HNU-DHK-2002-038.

\section{Paratypes $(3 \hat{\jmath} \hat{\partial}, 23$ 우 $)$}

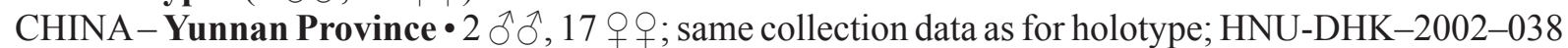
- 1 क; Cikai Township, Dabadi, North bank of Pula river; $27.78333^{\circ}$ N, $98.51667^{\circ}$ E; alt. 3030 m; 28 Sep. 2002; Heng-mei Yan leg.; HNU-Yan020928 • 1 क; Cikai Township, Dabadi to Gongshan, along Pula river downward; $27.7945833^{\circ} \mathrm{N}, 98.5071389^{\circ} \mathrm{E}$; alt. 3025-3990 m; 4 Oct. 2002; Heng-mei Yan leg.; HNU-Yan021004 - 1 \%; Lushui County, Waluoyaku; $25.78333^{\circ} \mathrm{N}, 98.78333^{\circ}$ E; alt. $1070 \mathrm{~m}$; 14 Oct. 2002; Heng-mei Yan leg.; HNU-Yan021014 • 2 o ㅇ; Tengchong County, Wuhe Township, Tongjia 12-dauzhuang Village, Longchuanjiang River (Longjiang bridge); $24.89284^{\circ} \mathrm{N}, 98.67439^{\circ} \mathrm{E}$; alt. 1210 m; 24 May 2005; Heng-mei Yan and Ke-ji Guo leg.; HNU-GKJ020 • 1 §̊; Nujiang Prefecture, Nujiang State Nature Reserve, No. 12 bridge Camp area, $16.3 \mathrm{~km} \mathrm{~W}$ of Gongshan; $27.71503^{\circ} \mathrm{N}$, 98.50244 ${ }^{\circ}$ E; alt. 2775 m; 15-19 Jul. 2000; Heng-mei Yan, D.H. Kavanaugh, Charles Griswold, Hongbin Liang, Darrell Ubick and Da-zhi Dong leg.; HNU-00-QD • 1 \%; Nujiang Prefecture, Nujiang State Nature Reserve, Dulong/Gongshan Yakou area, $21 \mathrm{~km}$ W of Gongshan; $27.69655^{\circ} \mathrm{N}, 98.45389^{\circ} \mathrm{E}$; alt. 3300-3680 m; 16-17 Jul. 2000; Heng-mei Yan, D.H. Kavanaugh, Charles Griswold, Hong-bin Liang, Darrell Ubick and Da-zhi Dong leg.; HNU-00-BY.

\section{Description}

\section{Male (holotype)}

Measurements and COlour. Total length: 2.95. Carapace 1.41 long, 1.14 wide, cephalothorax brown; clypeus 0.56 high (Fig. 14A-B); abdomen 1.54 long, 1.02 wide (Fig. 14A-B). Eye sizes and interdistances: AME 0.06, ALE 0.07, PME 0.07, PLE 0.07, AME-AME 0.04, PME-PME 0.06, AMEALE 0.08, PME-PLE 0.07, ALE-ALE 0.42, PLE-PLE 0.46. 

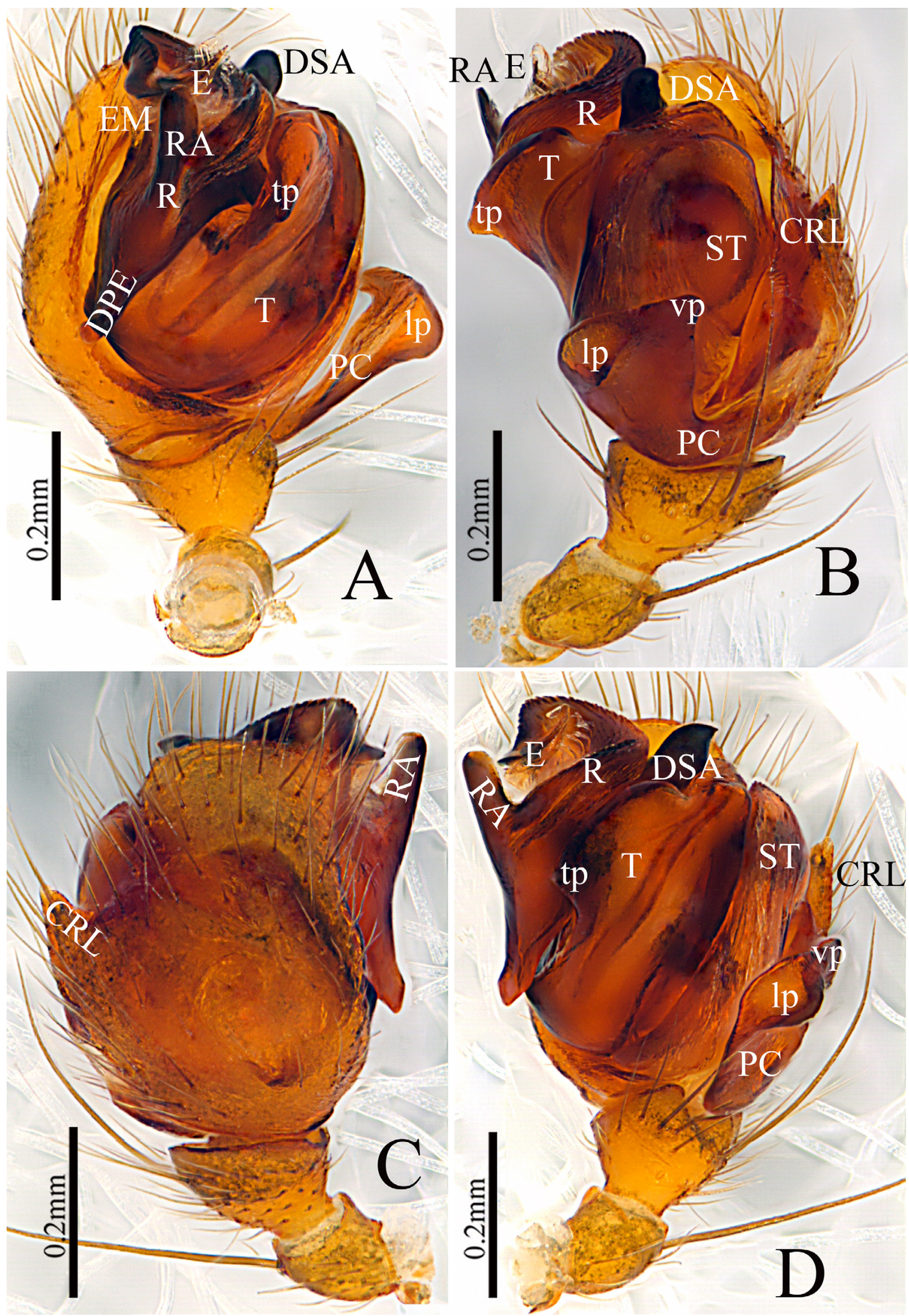

Fig. 10. Acroterius camur gen. et sp. nov., §, holotype (HNU-DHK-2002-038), palp. A. Prolateral view. B. Retrolateral view. C. Dorsal view. D. Ventral view. 

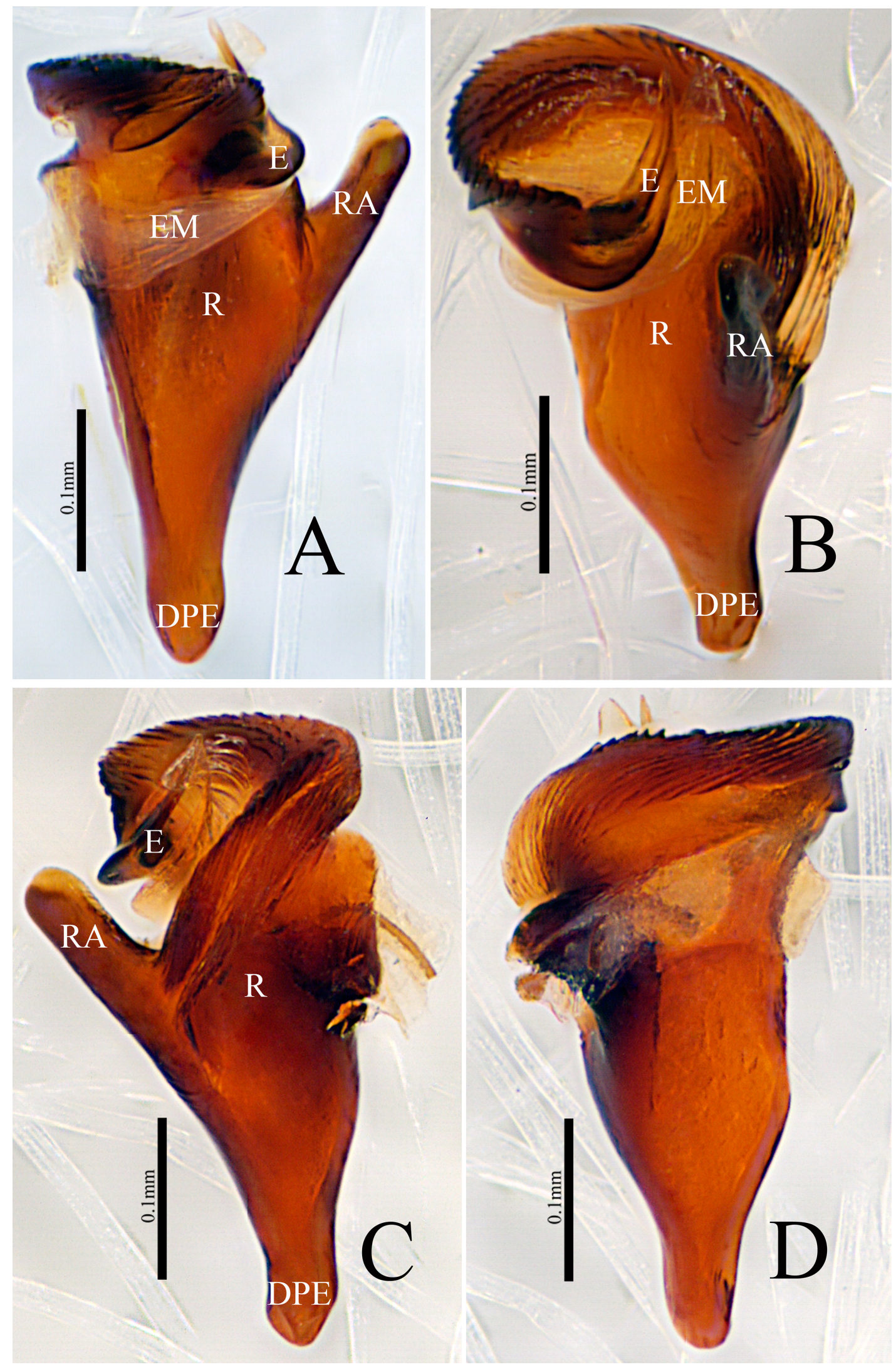

Fig. 11. Acroterius camur gen. et sp. nov., Ō, holotype (HNU-DHK-2002-038). A-B. Embolic division, ventral view. C. Embolic division, prolateral view. D. Embolic division, dorsal view. 
Chelicerae. With five promarginal and five retromarginal teeth (Fig. 14B); proximal two teeth of reteromargin much longer than distal one.

LENGTH OF LEGS. I $=6.11(1.65,1.92,1.59,0.95), \mathrm{II}=5.43(1.62,1.58,1.38,0.85), \mathrm{III}=4.13(1.19,1.26$, $1.05,0.63), \mathrm{IV}=5.61(1.58,1.79,1.32,0.92)$. Leg formula I-IV-II-III.

Palp (Figs 10A-D, 11A-D, 12A-B). Paracymbium highly sclerotized, distal arm with two projections; lateral projection small, somewhat triangular; ventral projection small with blunt end (Figs 10B, 12B); distal suprategular apophysis long, robust, distal part slightly curved with pointed end (Figs 10A-C, 12A-B). Radical apophysis thumb-shaped with blunt end extending above the embolus in ventral view (Figs 10A-D, 11A-D, 12A-B). Embolus stout, short with pointed end (Figs 10A-D, 11A-D, 12A-B).

Female (HNU-DHK-2002-038)

Measurements AND COLOUR. Total length: 2.66. Carapace 1.17 long, 1.01 wide, cephalothorax brown; clypeus 0.24 high (Fig. 14C-D); abdomen 1.49 long, 1.21 wide (Fig. 14C-D). Eye sizes and interdistances: AME 0.05, ALE 0.09, PME 0.08, PLE 0.07, AME-AME 0.04, PME-PME 0.06, AMEALE 0.06, PME-PLE 0.05, ALE-ALE 0.41, PLE-PLE 0.46, ALE-PLE contiguous.
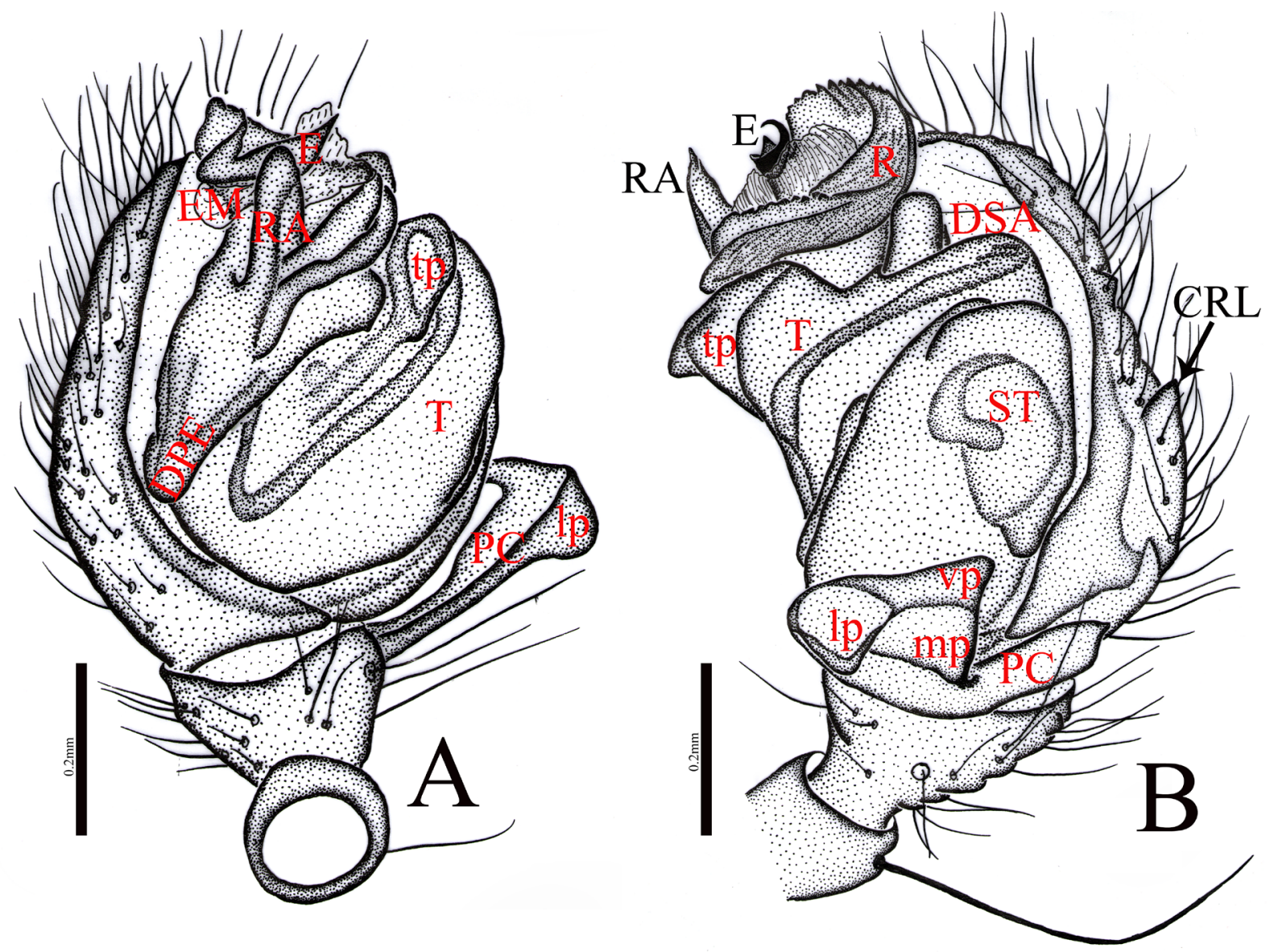

Fig. 12. Acroterius camur gen. et sp. nov., $\widehat{\jmath}$, holotype (HNU-DHK-2002-038), palp. A. Prolateral view. B. Retrolateral view. 
Chelicerae. With five promarginal and six retromarginal teeth; proximal teeth of reteromargin are much longer than distal one.

LeNGTH of Legs. I $=4.73(1.31,1.54,1.06,0.82), \mathrm{II}=4.13(1.28,1.23,1,0.62), \mathrm{III}=3.45(0.99,1.07$, $0.91,0.48), \mathrm{IV}=4.41(1.29,1.32,1.02,0.78)$. Leg formula I-IV-II-III.

Epigyne. Parmula 0.21 long, 0.41 wide at base (Fig. 13A-B); the posterior margin of anterior wall of epigynal plate with a projection horizontal to anterior wall of epigynal plate margin in orientation (Fig. 13A-B). Spermathecae slender, distal part hook-shaped (Fig. 13D).

\section{Distribution}

China (Yunnan Province, Fig. 38).
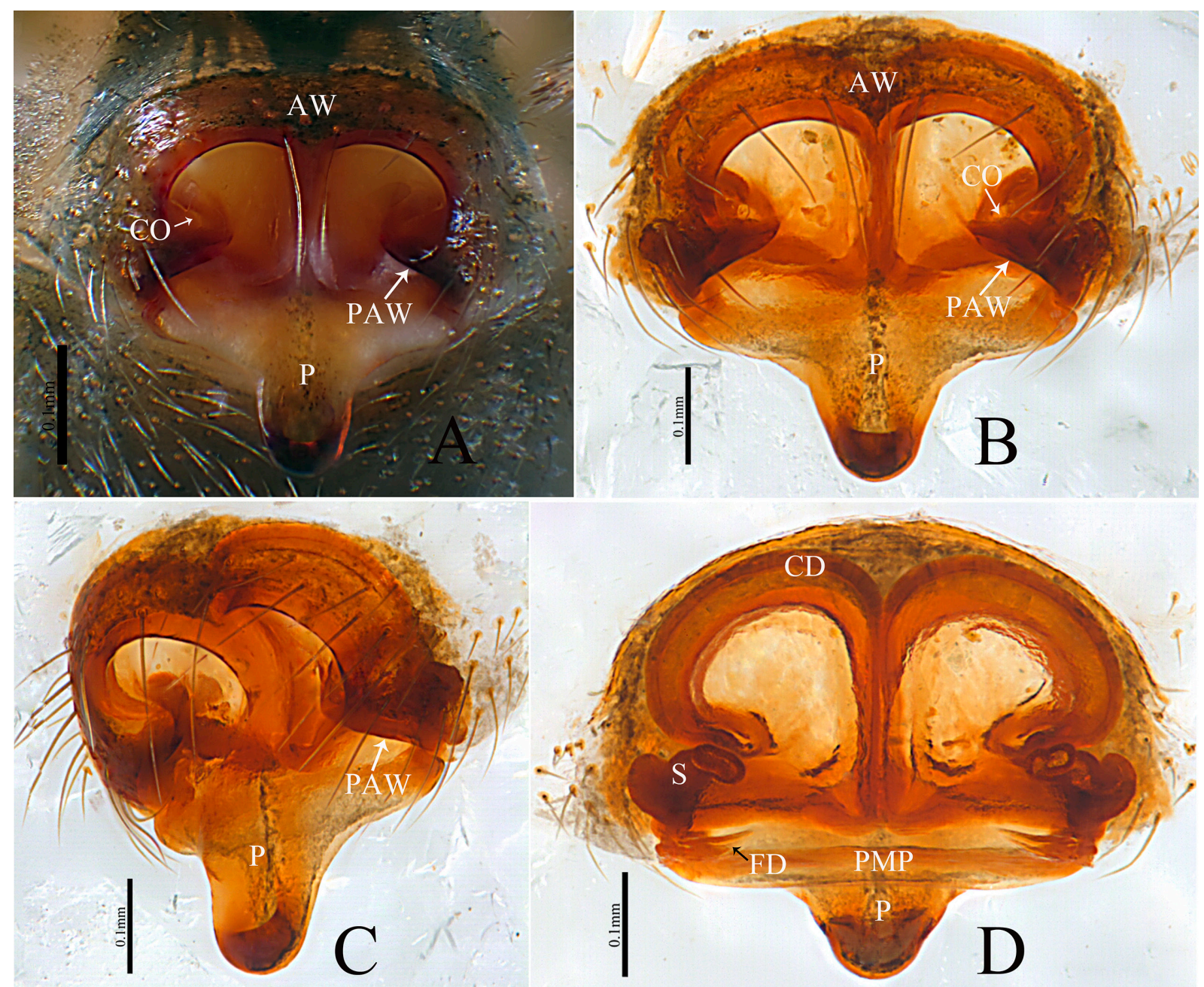

Fig. 13. Acroterius camur gen. et sp. nov.,, , paratype (HNU-DHK-2002-038). A-B. Epigyne, ventral view. C. Epigyne, lateral view. D. Vulva, dorsal view. 

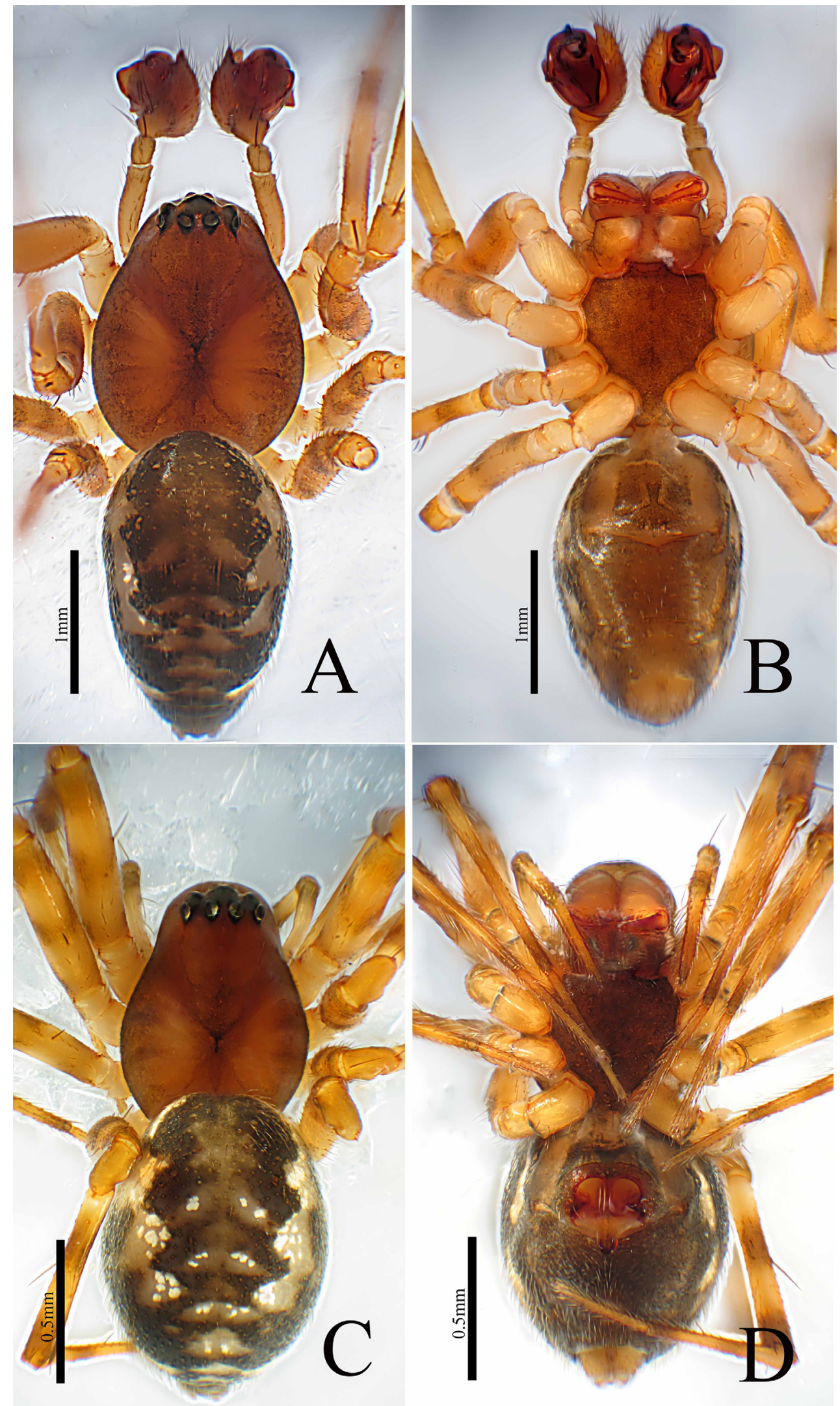

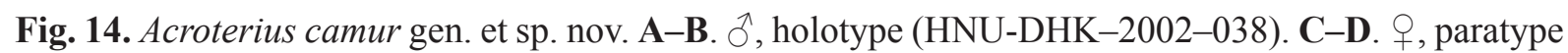
(HNU-DHK-2002-038). A, C. Habitus, dorsal view. B, D. Habitus, ventral view. 


\section{Acroterius circinatus gen. et sp. nov. urn:1sid:zoobank.org:act:826A098B-3362-42BB-95F7-3E3E0C763F04}

Figs 15-18, 39

\section{Differential diagnosis}

The new species resembles Acroterius brevis gen. et sp. nov. (Figs 6A-D, 7A-B, 8A-D) in having a long distal suprategular apophysis and radix with broad circular serrated part, which give rise to the embolus at its distal end in the male palp, parmula with socket on ventral surface and copulatory ducts forming a semicircular loop before joining the spermathecae in epigyne (Figs 15A-D, 16A-B, 17A-D); it can be distinguished by the median projection of the distal arm of paracymbium wider than long (Figs 15B, 16B), but longer than wide in A. brevis gen. et sp. nov. (Figs 6B, 7B). The embolus basally broad, disk-shaped, robust and almost touches the semicircular serrated part of the radix (Figs 15A-D, 16A-B), but stout, thread-like and pointing away from the semicircular serrated part of the radix in A. brevis gen. et sp. nov. (Figs 6A-D, 7A-B). Radical apophysis thumb-shaped, curved (Figs 15B, 16B), but somewhat triangular in A. brevis gen. et sp. nov. (Figs 6B, 7B). The spermathecae situated dorsomesally (Fig. 17D), but situated dorso-laterally in A. brevis gen. et sp. nov. (Fig. 8D).

\section{Etymology}

The species name comes from the Latin adjective 'circinatus', meaning 'circular' and referring to the circinate-shaped copulatory ducts in the ventral view of the epigyne.

\section{Type material}

\section{Holotype}

CHINA - O'; Yunnan Province, Longyang County, Bawan Township, Sancha He, Luoshuidong area; 24.92597 N, 98.75806 E; alt. 2300 m; 3 Jun. 2005; Charles Griswold leg.; HNU-CGY137.

\section{Paratypes $(9 \hat{\partial} \widehat{\partial}, 5$ \& $\odot)$}

CHINA - Yunnan Province, Longyang County -7 same collection data as for holotype;

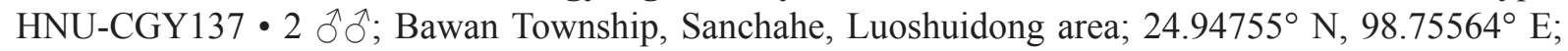
alt. 2300 m; 3 Jun. 2005; Heng-mei Yan, Ke-ji Guo and Hong-bin Liang leg.; HNU-GKJ039 • 3 우웅 Bawan Dist., Nankang Yakou; 24.83178 ${ }^{\circ}$ N, 98.76472 ${ }^{\circ}$ E; alt. 2180 m; 25 May 2005; Charles Griswold and D.H. Kavanaugh leg.; HNU-2005-029A • 2 우; Bawan Township, Dasheyao Yakou; $24.93167^{\circ} \mathrm{N}$, 98.75368 E; alt. $2488 \mathrm{~m}$; on top of mountain; 30 May 2005; Heng-mei Yan and Ke-ji Guo leg.; HNU-CGY32.

\section{Description}

Male (holotype)

MeAsurements AND COLOUR. Total length: 3.25. Carapace 1.56 long, 1.24 wide, cephalic region strongly elevated, yellowish; fovea, cervical and radial grooves distinct; clypeus 0.41 high (Figs 18A-C); abdomen 1.69 long, 1.21 wide (Fig. 18A-C). Eye sizes and interdistances: AME 0.08, ALE 0.09, PME 0.08, PLE 0.09, AME-AME 0.04, PME-PME 0.05, AME-ALE 0.9, PME-PLE 0.12, ALE-ALE 0.44, PLE-PLE 0.48, ALE-PLE contiguous.

CHELICERAE. With five promarginal and five retromarginal teeth.

LENGTH OF LEGS. I $=6.57(1.71,2.03,1.88,0.95), \mathrm{II}=5.78(1.62,1.81,1.51,0.84), \mathrm{III}=4.43(1.29,1.33$, $1.13,0.68), \mathrm{IV}=5.63(1.64,1.68,1.49,0.82)$. Leg formula I-II-IV-III.

PalP (Figs 15A-D, 16A-B). Paracymbium highly sclerotized, distal part with three projections, lateral projection large, slightly curved, tongue-shaped with blunt end in ventral view; median projection 


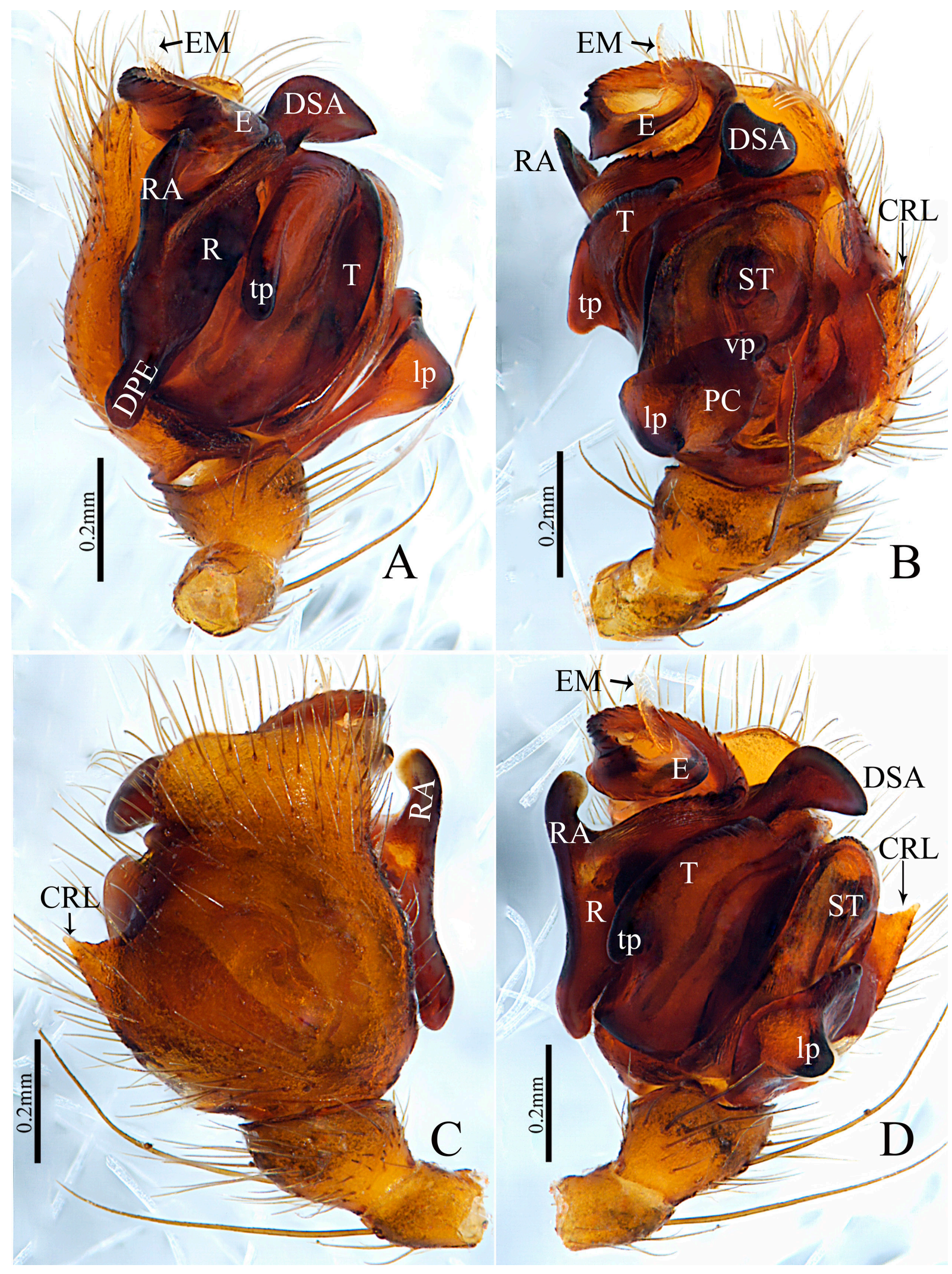

Fig. 15. Acroterius circinatus gen. et sp. nov., đ, holotype (HNU-CGY137), palp. A. Prolateral view. B. Retrolateral view. C. Dorsal view. D. Ventral view. 
wider than long; ventral projection sclerotized, small with blunt end, strongly overlapping subtegulum (Figs 15B, 16B); distal suprategular apophysis long, distal part curved and pointing towards tegulum (Figs 15A-D, 16A-B). Radical apophysis slightly curved with blunt end, protruding upward above base of embolus (Figs 15A-D, 16A-B); embolic membrane protruding above distal semicircular part, in prolateral view (Figs 15A, 16A). Embolus highly sclerotized, robust, disk-shaped, almost touching semicircular serrated part of radix with pointed end (Figs 15A-D, 16A-B).

\section{Female (HNU-CGY137)}

MEASUREMENTS AND COLOUR. Total length: 3.38. Carapace 1.42 long, 1.12 wide, yellowish; fovea, cervical and radial grooves distinct; clypeus 0.19 high (Fig. 18D-F); abdomen 1.96 long, 1.61 wide (Fig. 18DF). Eye sizes and interdistances: AME 0.07, ALE 0.09, PME 0.09, PLE 0.09, AME-AME 0.05, PMEPME 0.06, AME-ALE 0.07, PME-PLE 0.11, ALE-ALE 0.45, PLE-PLE 0.51, ALE-PLE contiguous.

Chelicerae. With six promarginal and seven retromarginal teeth; reteromarginal teeth gradually decrease in length towards the distal end.

LENGTH OF LEGS. I $=5.32(1.37,1.69,1.32,0.94), \mathrm{II}=4.75(1.36,1.42,1.15,0.82), \mathrm{III}=3.43(1.04,1.04$, $0.79,0.56), \mathrm{IV}=4.58(1.26,1.44,1.12,0.76)$. Leg formula I-II-IV-III.
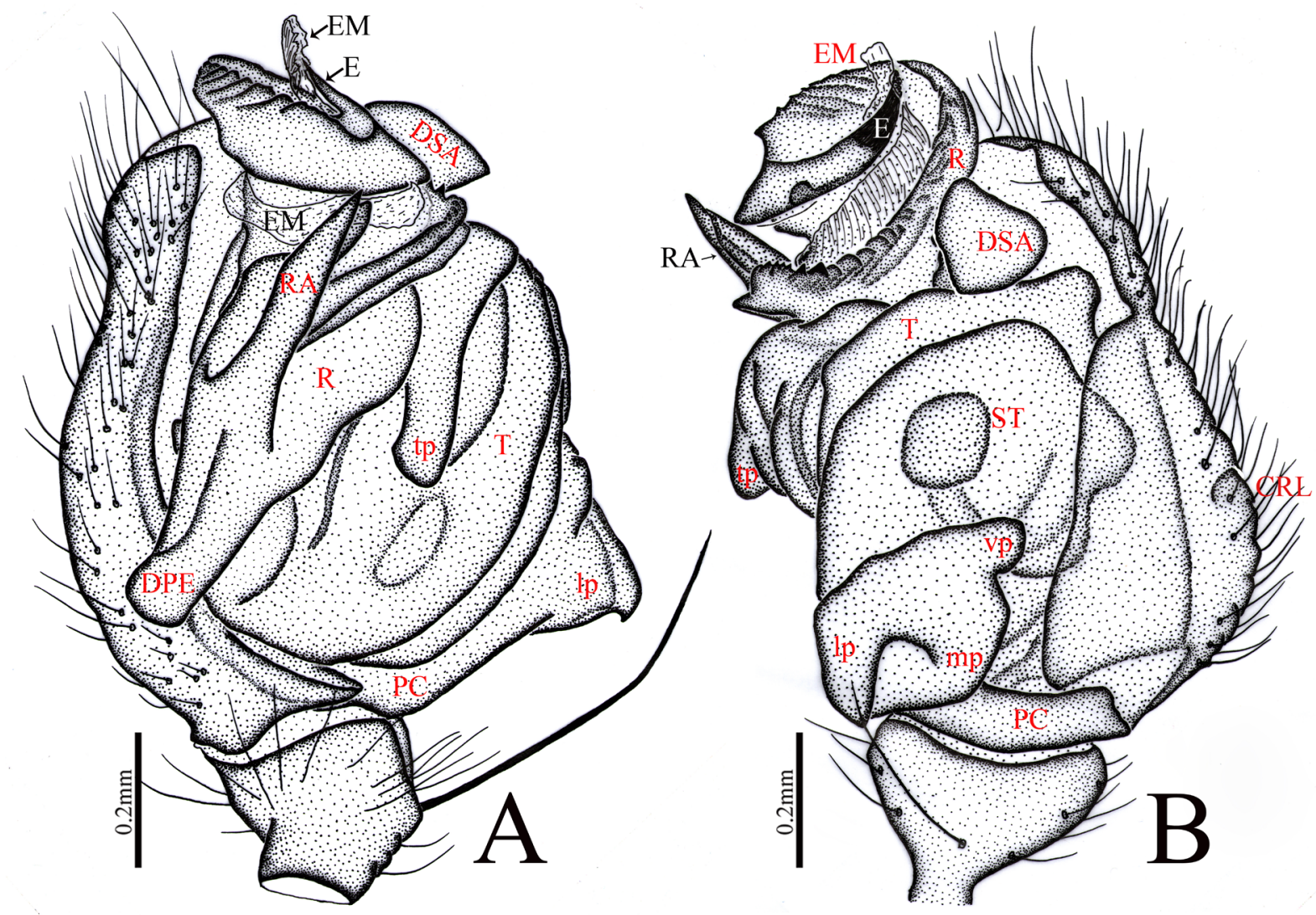

Fig. 16. Acroterius circinatus gen. et sp. nov., §̂, holotype (HNU-CGY137), palp. A. Prolateral view. B. Retrolateral view. 
Epigyne. Parmula 0.55 long, 0.24 wide at base (Fig. 17A-B); posterior projection of anterior wall of epigynal plate circular, forming circinate appearance (Fig. 17A-B). Spermathecae slender, situated dorso-mesally near median septum pointing toward lateral walls (Fig. 17D).

\section{Distribution}

China (Yunnan Province, Fig. 39).
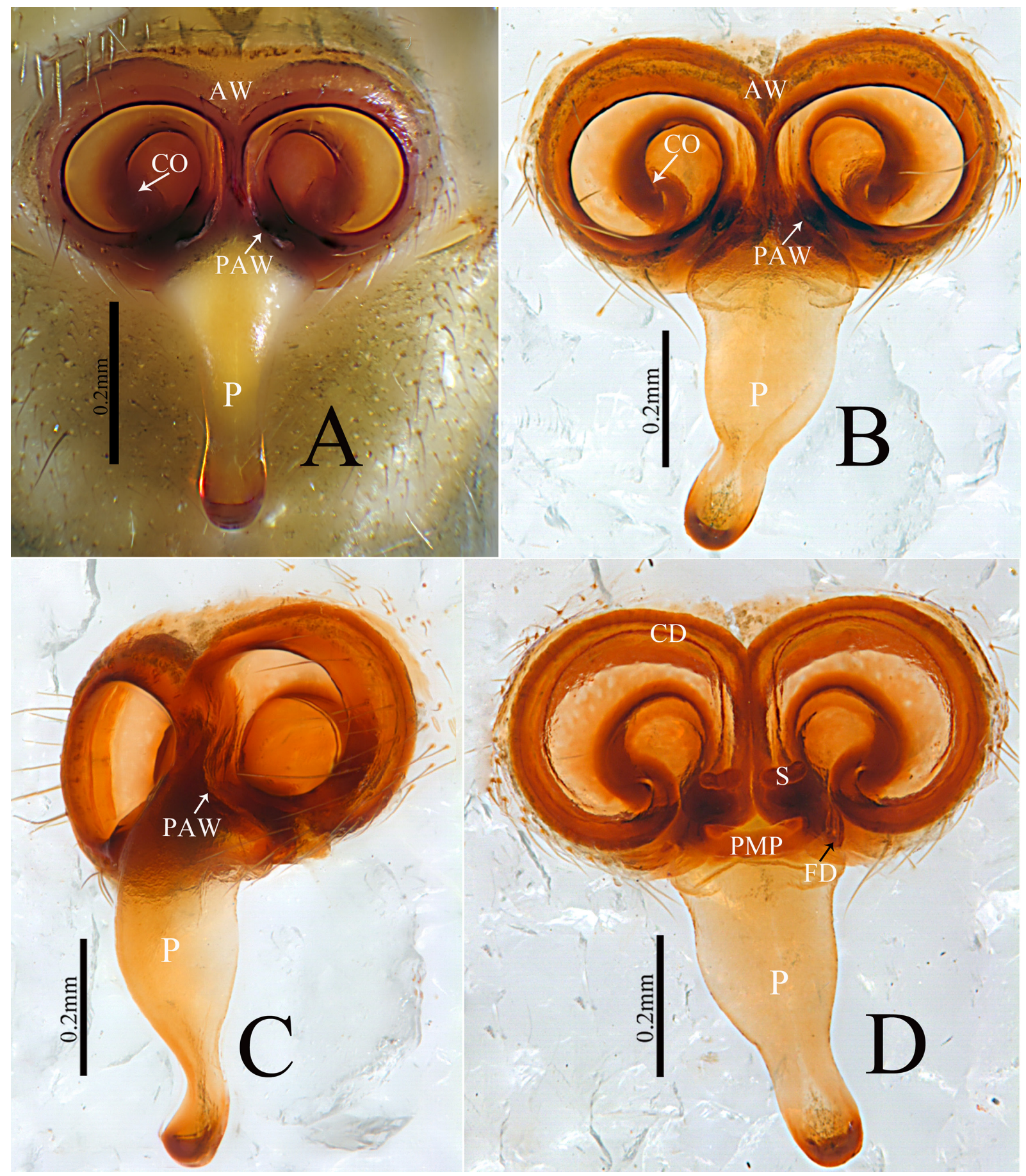

Fig. 17. Acroterius circinatus gen. et sp. nov., $q$, paratype (HNU-CGY137). A-B. Epigyne, ventral view. C. Epigyne, lateral view. D. Vulva, dorsal view. 


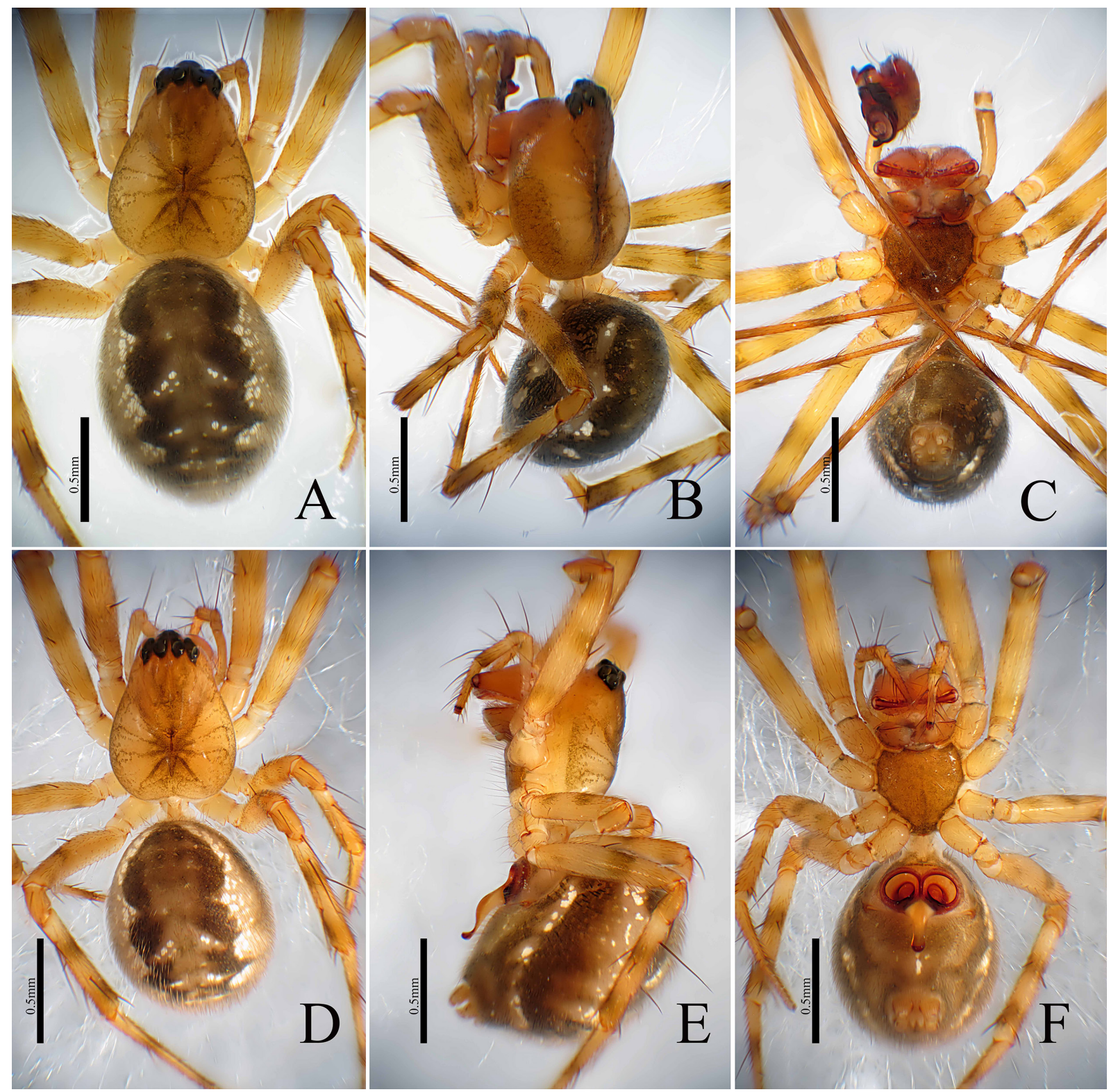

Fig. 18. Acroterius circinatus gen. et sp. nov. A-C. $\overbrace{}^{\lambda}$, holotype (HNU-CGY137). D-F. + , paratype (HNU-CGY137). A, D. Habitus, dorsal view. B, E. Habitus, lateral view. C, F. Habitus, ventral view.

Acroterius hamatus gen. et sp. nov.

urn:1sid:zoobank.org:act:754D348A-8F5C-47DA-9940-FDCEDD520F56

Figs 19-21, 38

\section{Differential diagnosis}

The new species resembles Acroterius brevis gen. et sp. nov. (Fig. 8A-D) in having the posterior median plate extending posteriorly with a parmula, but can be distinguished by: in dorsal view, parmula two times the width of parmula base, with distal part distinctly expanded (Fig. 19D), slightly longer than the 
width of parmula base (Fig. 19A-D), but distal part not expanded in A. brevis gen. et sp. nov. In lateral view, anterior wall of epigynal plate projection wider than long (Figs 19C-21C), but longer than wide in A. brevis gen. et sp. nov. (Fig. 8C).
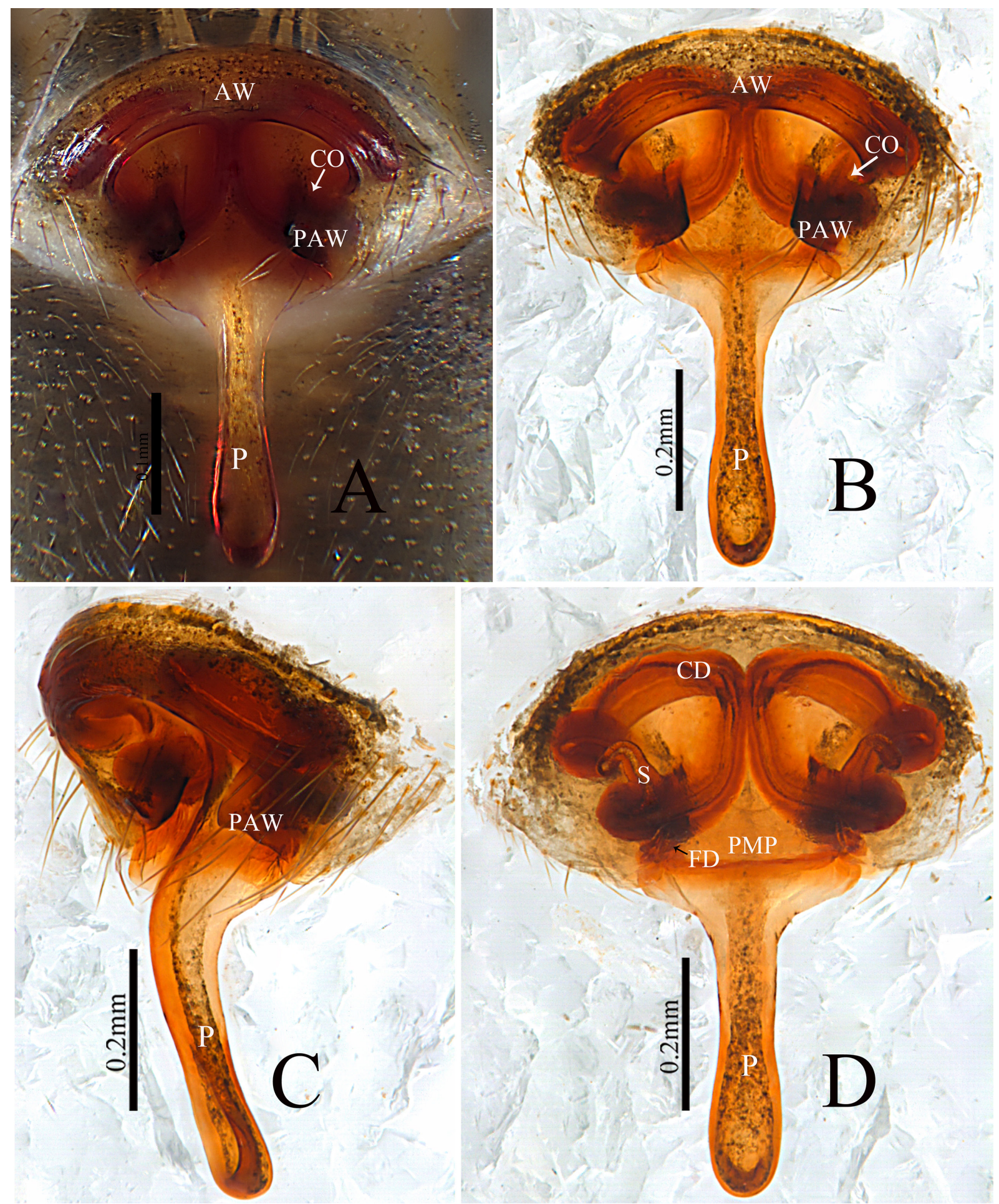

Fig. 19. Acroterius hamatus gen. et sp. nov., + , holotype (HNU-Tang-04-04). A-B. Epigyne, ventral view. C. Epigyne, lateral view. D. Vulva, dorsal view. 


\section{Etymology}

The species name comes from the Latin adjective 'hamatus', meaning 'hook' and referring to the hookshaped spermathecae in the epigyne.

\section{Type material}

\section{Holotype}

CHINA - O; Yunnan Province, Gongshan County, Dulongjiang Township, Kongdang Village; $27.87961^{\circ} \mathrm{N}, 98.33878^{\circ} \mathrm{E}$; alt. 1527 m; 25 Oct. 2004; Guo Tang leg.; HNU-Tang-04-04.

\section{Paratypes $(5$ 우)}

CHINA - Yunnan Province, Gongshan County - 4 우; $77 \mathrm{~km}$ of new road to Donglongjiang; 27.91034 ${ }^{\circ}$ N, 98.41081 ${ }^{\circ}$ E; alt. 2183 m; 8-9 Nov. 2004; Guo Tang leg.; HNU-Tang-04-09 • 1 O; Dulongjiang Township, S/N of Dizhengdang Village along Silalong He; $28.07654^{\circ} \mathrm{N}, 98.32603^{\circ} \mathrm{E}$; alt. 1890 m; 28 Oct. 2004; D.H. Kavanaugh leg.; HNU-DHK-2004-059.

\section{Description}

Female (holotype)

Measurements And COlour. Total length: 3.43 . Carapace 1.41 long, 1.17 wide, cephalothorax brown; clypeus 0.41 high (Fig. 20A-B); abdomen 2.01 long, 1.41 wide. Eye sizes and interdistances:

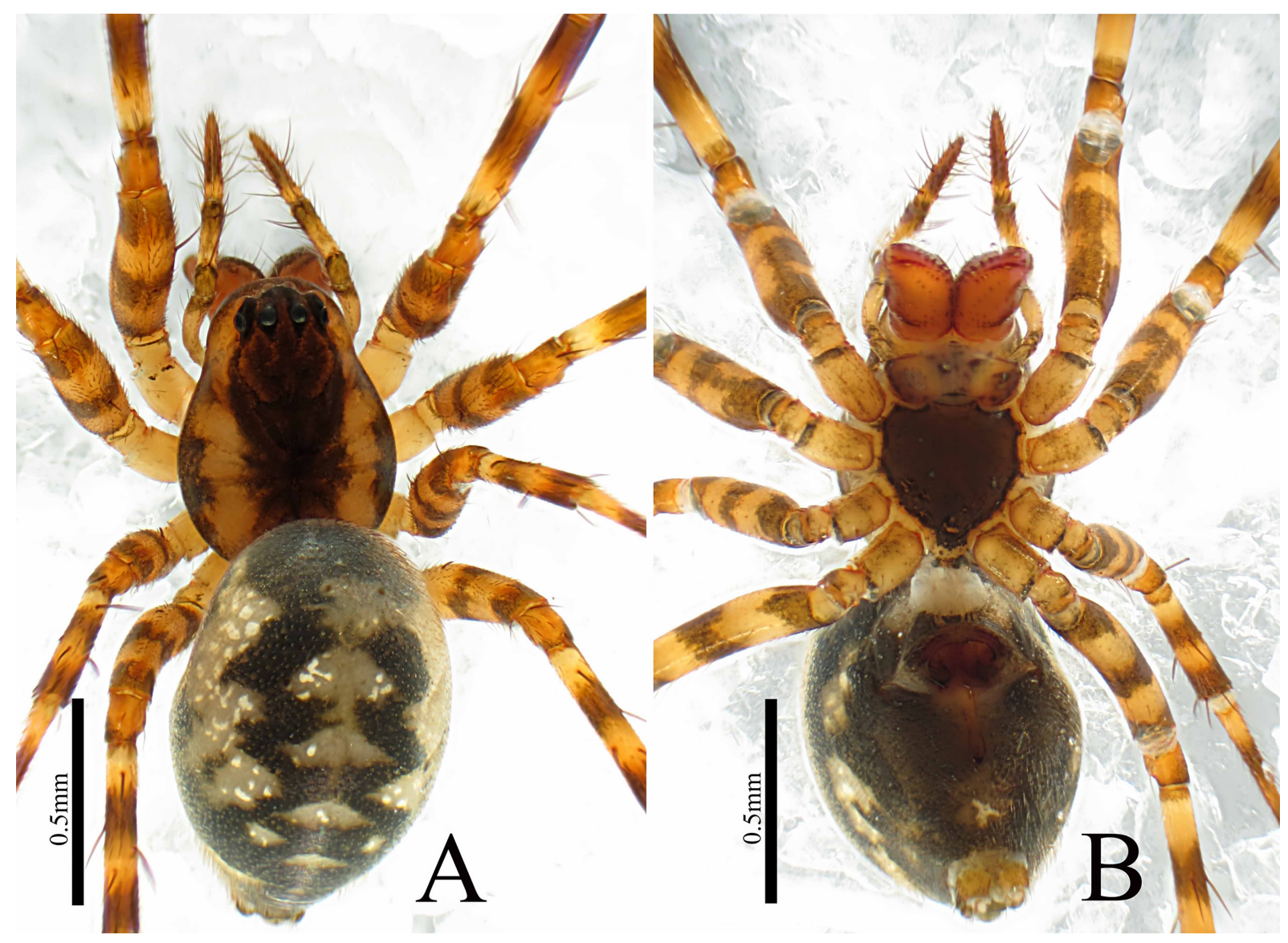

Fig. 20. Acroterius hamatus gen. et sp. nov., + , holotype (HNU-Tang-04-04). A. Habitus, dorsal view. B. Habitus, ventral view. 

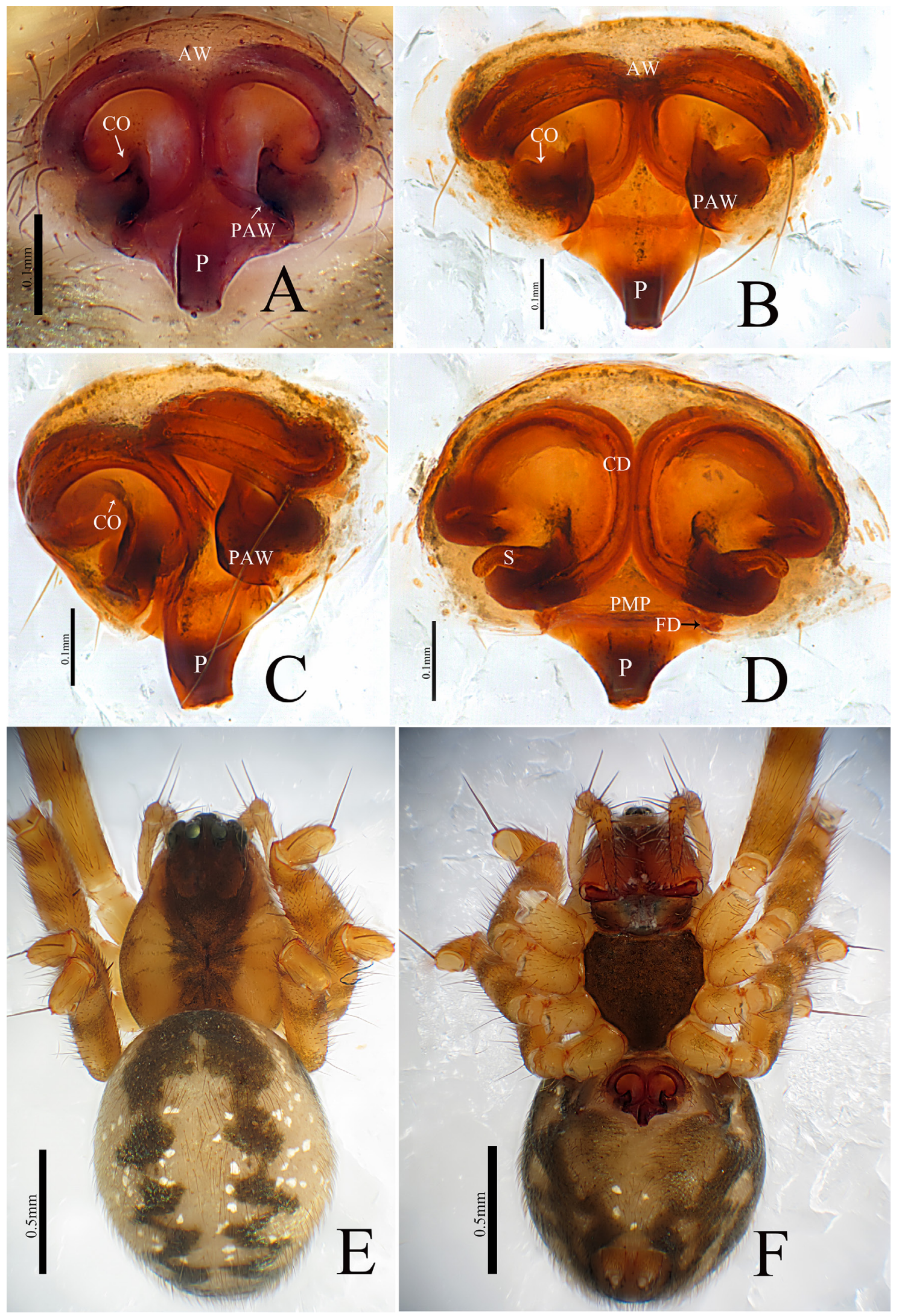

Fig. 21. Acroterius hamatus gen. et sp. nov., + , paratype (HNU-Tang-04-09). A-B. Epigyne, ventral view. C. Epigyne, lateral view. D. Vulva, dorsal view. E. Habitus, dorsal view. F. Habitus ventral view. 
AME 0.06, ALE 0.08, PME 0.07, PLE 0.07, AME-AME 0.04, PME-PME 0.07, AME-ALE 0.09, PME-PLE 0.08, ALE-ALE 0.49, PLE-PLE 0.53, ALE-PLE contiguous.

Chelicerae. With six promarginal and six retromarginal teeth; reteromarginal teeth long and highly sclerotized protruding beyond fangs.

LeNGTH OF LEGs. I $=5.39(1.74,1.40,1.31,0.94), \mathrm{II}=4.99(1.42,1.54,1.17,0.83), \mathrm{III}=3.95(1.18,1.25$, $0.9,0.62), \mathrm{IV}=4.94(1.44,1.53,1.18,0.79)$. Leg formula I-II-IV-III.

Epigyne. Parmula 0.53 long, 0.28 wide at base (Fig. 19A-C); posterior margin of anterior wall of epigynal plate with projection wider than long on each side (Fig. 19A-B). Spermathecae slender, distal part slightly curved, pointing towards posterior margin of epigyne (Fig. 19D).

VARIATION IN the EPIGYNe (HNU-Tang-04-09, Fig. 21A-F). We noticed in the four out of five female paratypes that the epigynal parmulas are broken at the same point due to unknown reasons. All the other somatic and genitalic characters are found to be similar (Fig. 21A-D).

\section{Male}

Unknown.

\section{Distribution}

China (Yunnan Province, Fig. 38).

Acroterius inversus gen. et sp. nov. urn:1sid:zoobank.org:act:42879150-7E48-4E0F-8C7B-004DDB375847

Figs 22-23, 38

\section{Differential diagnosis}

The new species resembles Acroterius camur gen. et sp. nov. (Fig. 13A-D) in having the similar short parmula in epigyne as in A. brevis gen. et sp. nov. and A. longidentatus gen. et sp. nov. (Fig. 22A-D); it can be distinguished by: spermathecae sinuous (Fig. 22D), but hook-shaped in A. camur gen. et sp. nov. (Fig. 13D). Parmula anteriorly rectangular and posteriorly spoon-shaped (Fig. 22A-C), but tongueshaped in A. brevis gen. et sp. nov. (Fig. 8A-C), somewhat triangular in A. camur gen. et sp. nov. (Fig. 13A-C), finger-shaped in A. longidentatus gen. et sp. nov. (Fig. 29A-C).

\section{Etymology}

The species name comes from the Latin adjective 'inversus', meaning 'inverted' and referring to the inverted L-shaped spermathecae in the epigyne.

\section{Type material}

\section{Holotype}

CHINA - + ; Yunnan Province, Lonyang County, Bawan Township, Nankang Yakou; $24.83178^{\circ} \mathrm{N}$, 98.76472 E; alt. 2180 m; 25 May 2005; Charles Griswold leg.; HNU-CGY115.

\section{Paratypes $(4$ + + $)$}

CHINA - Yunnan Province 2 2 $ᄋ$; Lonyang County, Baoshan City, Nankang Yakou (National 320 Road); $24.43717^{\circ} \mathrm{N}, 98.46054^{\circ} \mathrm{E}$; alt. 2186 m; 30 Oct. 2003; Guo Tang leg.; HNU-Tang031031 • 1 q; Lushui County, Pianma Township, Chanyan He, $9.3 \mathrm{~km}$ to Pianma; $25.99363^{\circ} \mathrm{N}, 98.66651^{\circ} \mathrm{E}$; 
alt. $2470 \mathrm{~m}$; mixed broadleaf deciduous and evergreen forest; 13-14 May 2005; Charles Griswold leg.; HNU-CGY105 • 1 क; Longling County, Longjiang Township, Xiaoheishan Nature Reserve; $24.82888^{\circ} \mathrm{N}, 98.76001^{\circ} \mathrm{E}$; alt. 2020 m; broadleaf forest; 26 May 2005; Charles Griswold leg.; HNUCGY124.
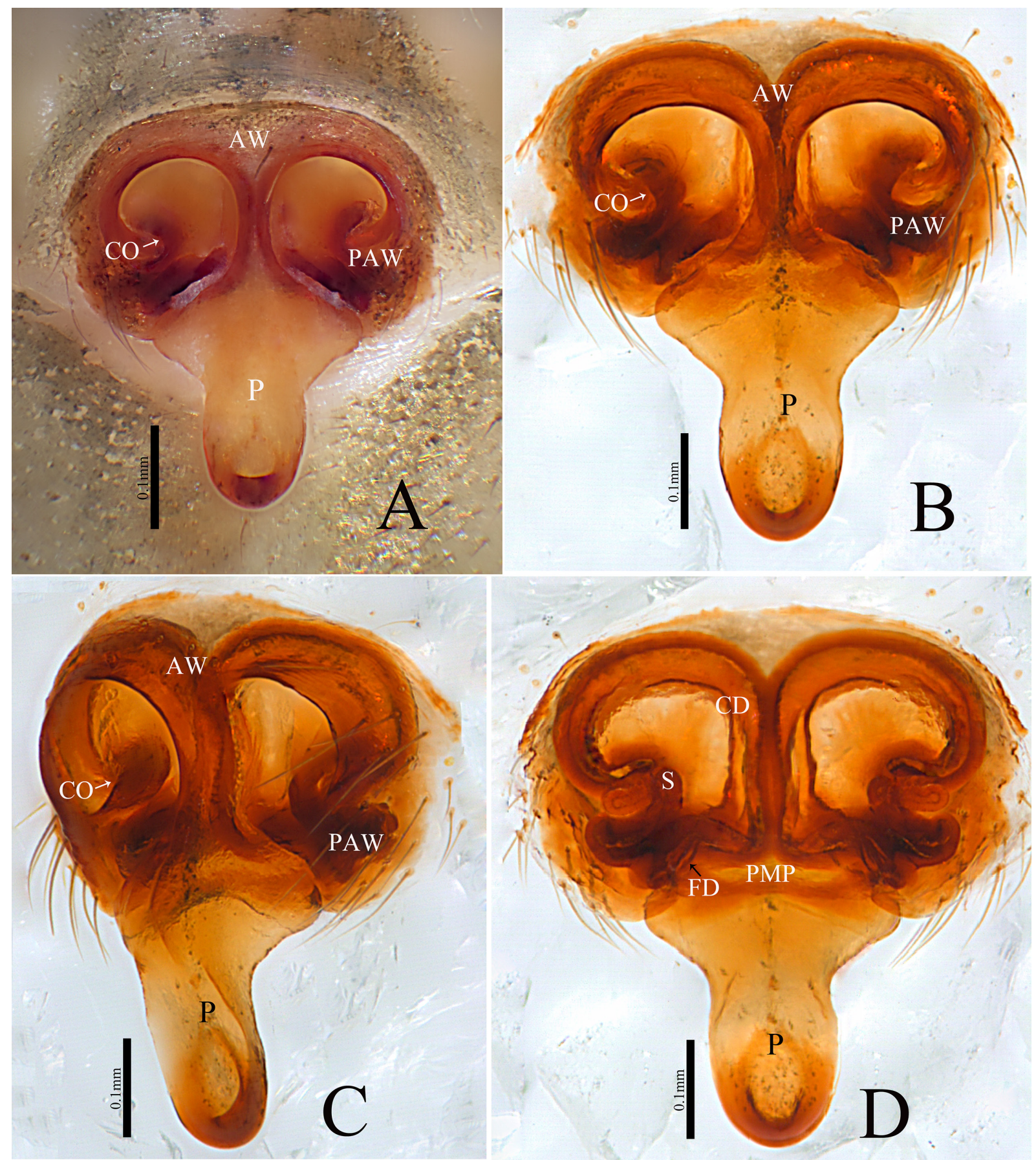

Fig. 22. Acroterius inversus gen. et sp. nov.,, , holotype (HNU-CGY115). A-B. Epigyne, ventral view. C. Epigyne, lateral view. D. Vulva, dorsal view. 


\section{Description}

Female (holotype)

MeAsurements AND COLOur. Total length: 3.33 . Carapace 1.10 long, 1.08 wide, cephalic region slightly elevated, yellowish to light brown; fovea, cervical and radial grooves distinct; clypeus 0.26 high (Fig. 23A-B); abdomen 2.22 long, 1.81 wide (Fig. 23A-B). Eye sizes and interdistances: AME 0.07, ALE 0.09, PME 0.08, PLE 0.07, AME-AME 0.04, PME-PME 0.06, AME-ALE 0.05, PME-PLE 0.11, ALE-ALE 0.44, PLE-PLE 0.48, ALE-PLE contiguous.

Chelicerae. With six promarginal and six retromarginal teeth.

LENGTH OF LEGS. I $=5.04(1.41,1.62,1.22,0.79), \mathrm{II}=4.54(1.37,1.41,1.05,0.71), \mathrm{III}=3.37(1.12,0.93$, $0.81,0.51), \mathrm{IV}=4.61(1.38,1.42,1.12,0.69)$. Leg formula I-IV-II-III.

EPIGYNe. Posterior median plate extends posteriorly with short parmula with spoon-like deep socket ventrally (Fig. 22A-B); parmula 0.35 long, 0.25 wide at base (Fig. 22A-B); posterior margin of anterior wall of epigynal plate wider than long, with somewhat circle-like projection (Fig. 22A-B); copulatory openings situated inside atrium between anterior wall and posterior median plate (Fig. 22A-B),
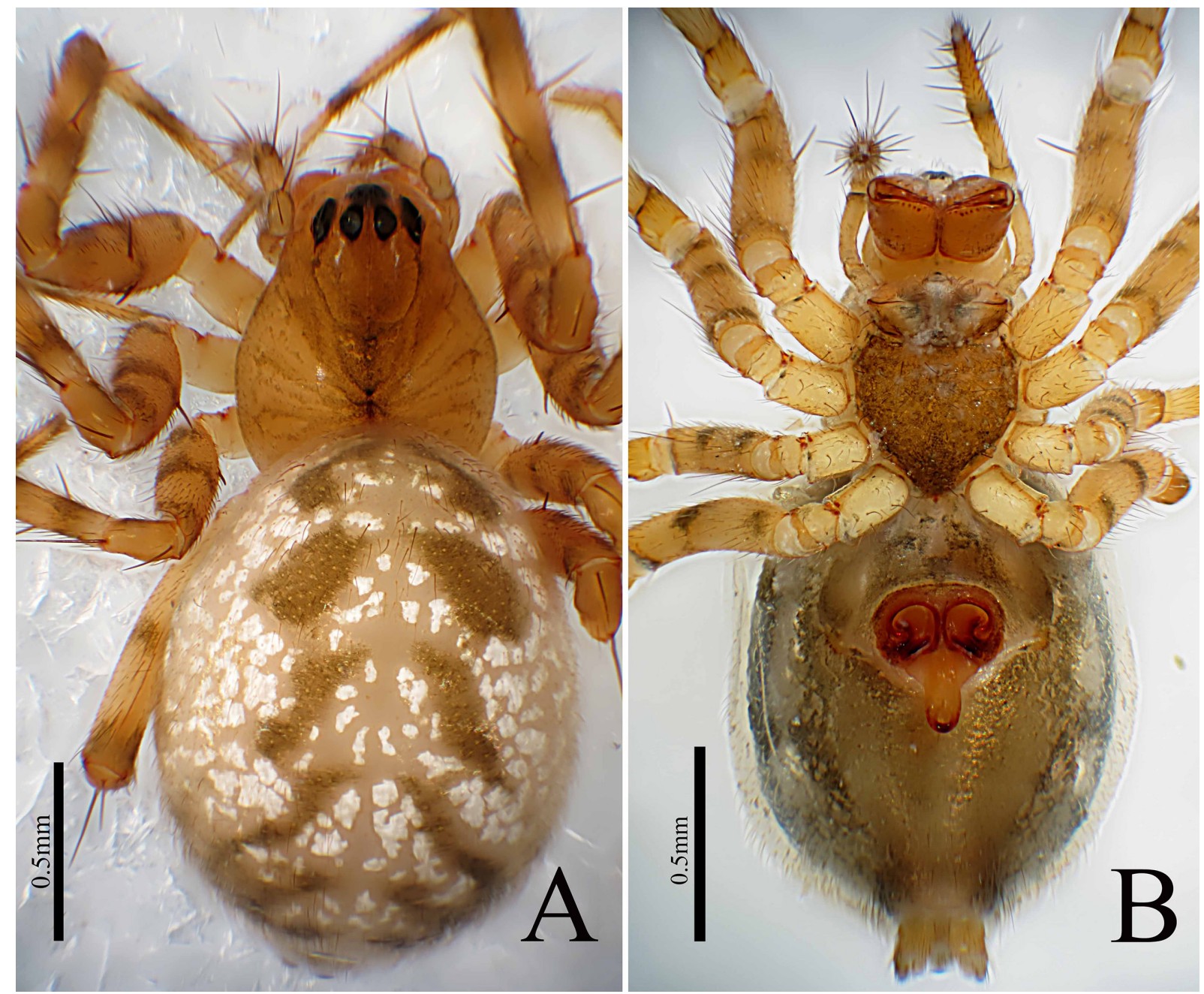

Fig. 23. Acroterius inversus gen. et sp. nov., $\wp$, holotype (HNU-CGY115). A. Habitus, dorsal view. B. Habitus, ventral view. 
connected with long, semicircular copulatory ducts joined to spermathecae (Fig. 22D). Spermathecae slender, sinuous, forming almost complete wave (Fig. 22D). Fertilization ducts present laterally on posterior median plate (Fig. 22D).

\author{
Male \\ Unknown. \\ Distribution \\ China (Yunnan Province, Fig. 38).
}

\author{
Acroterius latus gen. et sp. nov. \\ urn:lsid:zoobank.org:act:896C3469-C7F5-4931-ADBF-B423F6513F4A
}

Figs 24-28, 38

\title{
Differential diagnosis
}

The new species resembles Acroterius longimultus gen. et sp. nov. (Figs 30A-D, 31A-B) in having the distal arm of paracymbium with two projections in the male palp and the long parmula with a median incision in the epigyne (Figs 24A-D, 26A-B); it can be distinguished by: the distal suprategular apophysis short, retained below the apex of cymbium in retrolateral view (Fig. 24B), but long, swordshaped, protruding beyond the apex of cymbium in A. longimultus gen. et sp. nov. (Fig. 30B). The dorsal projection of embolic plate doesn't touch the base of cymbium in prolateral view (Figs 24A, 26A), but almost touches the base of cymbium in A. longimultus gen. et sp. nov. (Figs 30A, 31A). The lateral projection of the distal arm of the paracymbium is finger-shaped, almost touches the posterior margin of the tibia (Figs 24B, D, 26B), but is tongue-shaped and away from the posterior margin of the tibia in A. longimultus gen. et sp. nov. (Figs 30B, D, 31B). The posterior projection of the anterior wall of the epigynal plate is wider than long and doesn't protrude beyond the epigastric furrow (Fig. 27A-B), but is longer than wide and protruding beyond the epigastric furrow in A. longimultus gen. et sp. nov. (Fig. 32A-B). The distal part of the spermathecae is hook-shaped (Fig. 27D), but inverted L-shaped in A. longimultus gen. et sp. nov. (Fig. 32D).

\section{Etymology}

The species name comes from the Latin adjective 'latus' meaning 'broad', referring to the radical apophysis with a broad distal end in the male palp.

\section{Type material}

Holotype

CHINA - O'; Yunnan Province, Lonyang County, Bawan Distr., Nankang Yakou; $24.83178^{\circ} \mathrm{N}$, 98.76472 ${ }^{\circ}$ E; alt. 2180 m; 25 May 2005; Charles Griswold and D.H. Kavanaugh leg.; HNU-CGY123.

Paratypes $(3 q q)$

CHINA - Yunnan Province 1 1 ; same collection data as for holotype; HNU-CGY123 • 1 q; Fugong County, 2 km down Shibali; 27.16284 N, 98.78989 E; alt. 2420 m; 2 May 2004; Guang-xu Peng leg.; HNU-20040502 • 1 q; Fugong County, Lishadi Township, Shibali; 27.10520 N, $98.77980^{\circ}$ E; alt. 2530 m; 10 Aug. 2005; Guo Tang leg.; HNU-Tang-05-02.

\section{Description}

Male (holotype)

MeAsurements AND COLOUR. Total length: 3.55. Carapace 1.84 long, 1.47 wide, cephalic region slightly elevated, dark brown, with longitudinal band starting from base of posterior lateral eyes extending 

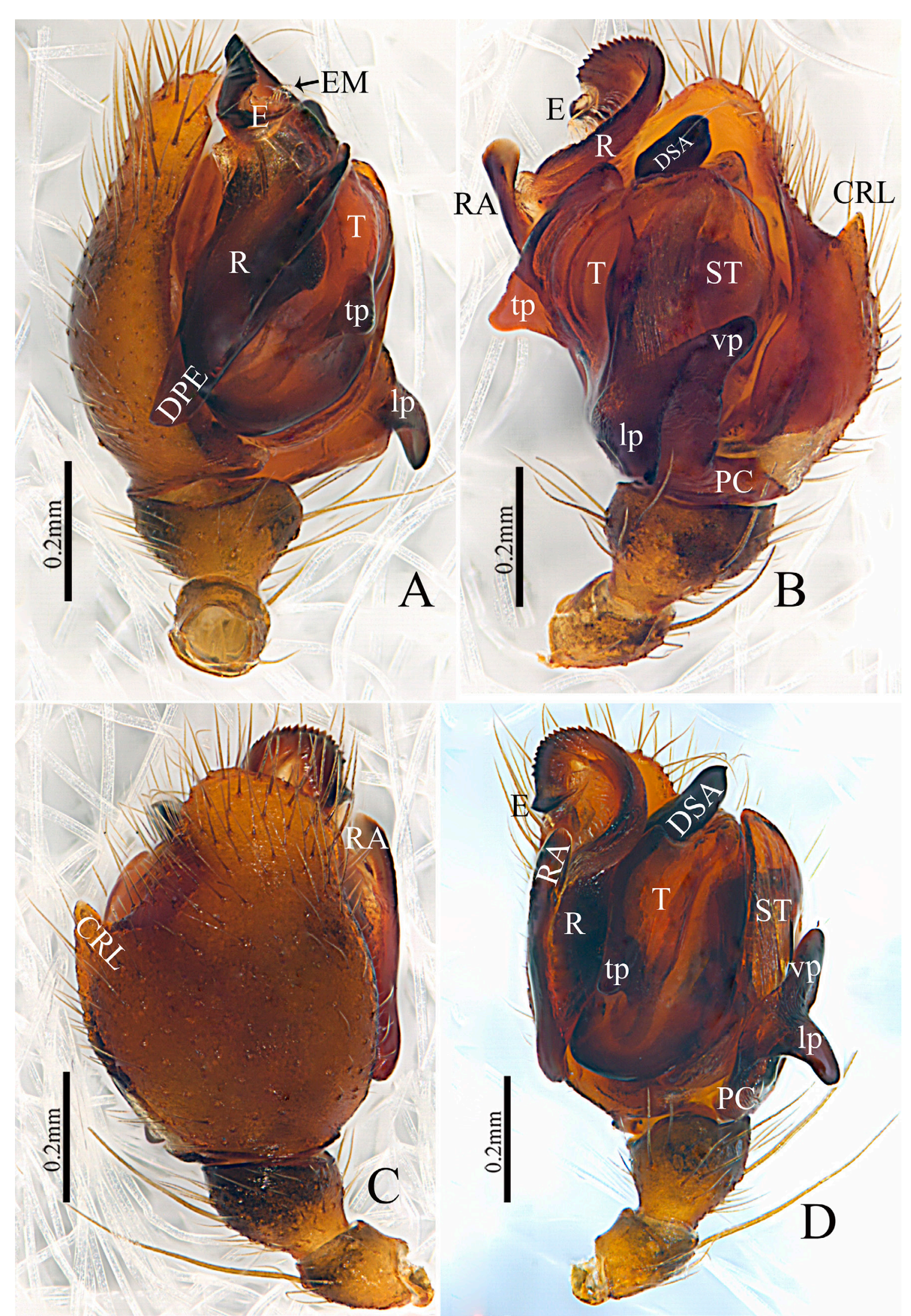

Fig. 24. Acroterius latus gen. et sp. nov., Ô, holotype (HNU-CGY123), palp. A. Prolateral view. B. Retrolateral view. C. Dorsal view. D. Ventral view. 
to base of cephalothorax followed by fovea, cervical and radial grooves distinct; clypeus 0.34 high (Fig. 28A-B); abdomen 1.71 long, 1.29 wide. Eye sizes and interdistances: AME 0.06, ALE 0.09, PME 0.07, PLE 0.07, AME-AME 0.07, PME-PME 0.09, AME-ALE 0.11, PME-PLE 0.13, ALE-ALE 0.51, PLE-PLE 0.55, ALE-PLE contiguous.

Chelicerae. With six promarginal and five retromarginal teeth; distal three teeth of promargin very small (Fig. 25B).

LeNGTH OF LEGS. I $=6.59(1.79,1.99,1.67,1.14), \mathrm{II}=4.88(1.66,1.69,1.32,1), \mathrm{III}=3.95(1.34,1.39$, $0.76,0.46), \mathrm{IV}=5.98(1.74,1.82,1.46,0.96)$. Leg formula I-IV-II-III.

PaLP (Figs 24A-D, 26A-B). Paracymbium highly sclerotized, distal arm with two projections (Figs 24B, 26B), lateral projection finger-like in ventral view and almost touches posterior margin of paracymbium in retrolateral view; ventral projection thumb-like with blunt end; distal suprategular apophysis slightly protruding above cymbium in ventral view (Figs 24D, 26B). Radical apophysis relatively large, tongueshaped, slightly curved with blunt end in ventral view (Figs 24A-D, 26A-B). Embolus stout, short with pointed end (Figs $24 \mathrm{~A}-\mathrm{C}, 26 \mathrm{~A}-\mathrm{B}$ ).

\section{Female (HNU-CGY123)}

MEASUREMENTS AND COLOUR. Total length: 3.03. Carapace 1.26 long, 1.01 wide, cephalic region slightly elevated, dark brown, with a longitudinal band starting from base of posterior lateral eyes extending to base of cephalothorax; fovea, cervical and radial grooves distinct; clypeus 0.25 high (Fig. 28C-D);

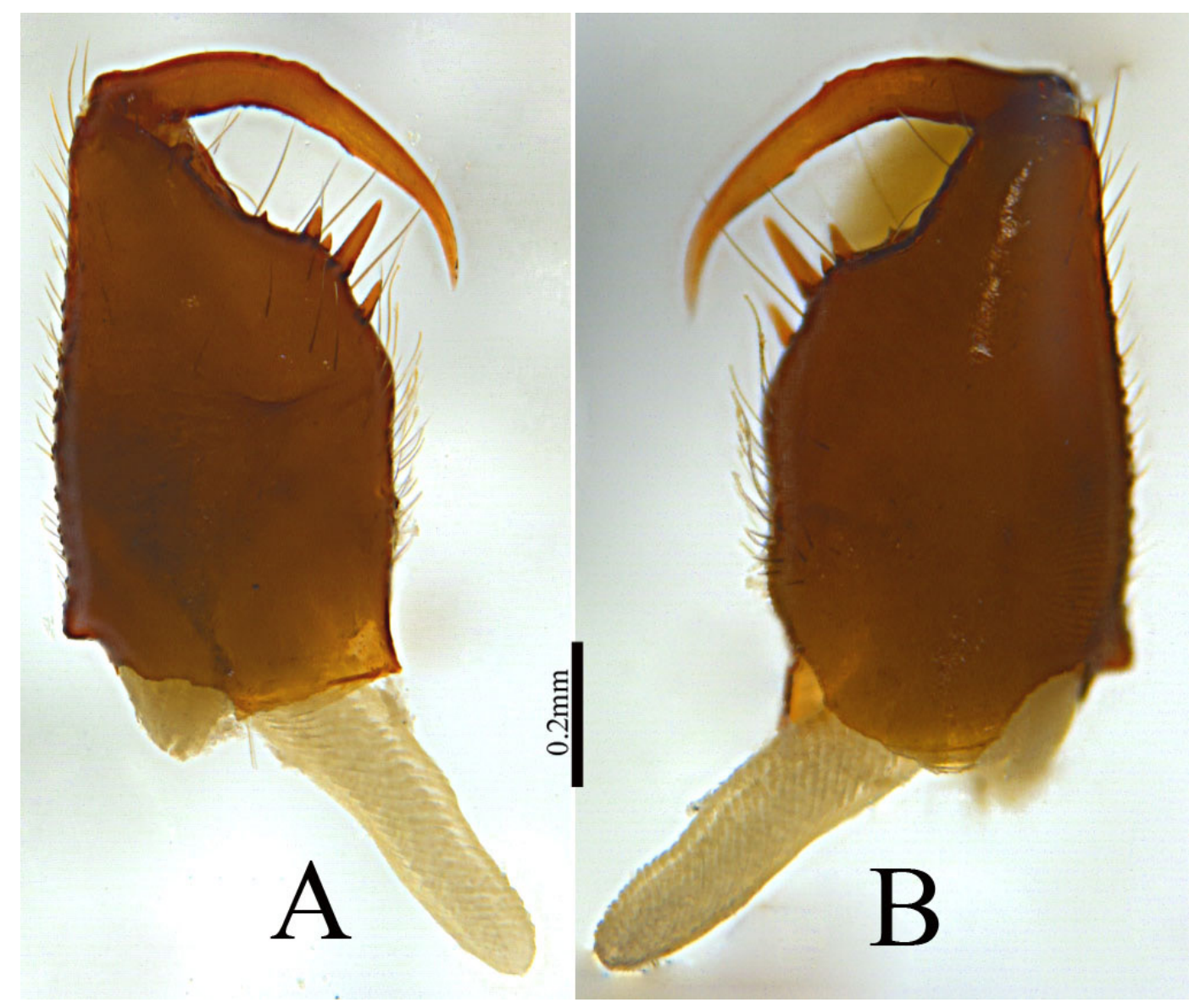

Fig. 25. Acroterius latus gen. et sp. nov., Ô, holotype (HNU-CGY123). A. Chelicera, dorsal view. B. Chelicera, ventral view. 
abdomen 1.75 long, 1.33 wide. Eye sizes and interdistances: AME 0.07, ALE 0.09, PME 0.07, PLE 0.08, AME-AME 0.05, PME-PME 0.10, AME-ALE 0.08, PME-PLE 0.07, ALE-ALE 0.43, PLE-PLE 0.47, ALE-PLE contiguous.

Chelicerae. In dorsal view, chelicerae with depression at proximal end slightly below reteromargin with six promarginal and seven retromarginal teeth; reteromarginal teeth gradually decreasing in length towards base of fang (Fig. 25A-B).

LENGTH OF LEGS. I $=4.30(1.29,1.48,0.89,0.64), \mathrm{II}=4.05(1.26,1.34,0.86,0.59), \mathrm{III}=2.27(1.02,1.06$, $0.69,0.52), \mathrm{IV}=4.39(1.23,1.34,1.03,0.79)$. Leg formula I-IV-II-III.

Epigyne. Parmula 0.45 long, 0.14 wide at base (Fig. 27A-B); posterior projection of anterior wall of epigynal plate margin of epigyne wider than long, not protruding beyond epigastric furrow (Fig. 27AB). Spermathecae slender, distal part of spermathecae hook-shaped, slightly curved, pointing towards lateral wall of epigyne (Fig. 27D).

\section{Distribution}

China (Yunnan Province, Fig. 38).
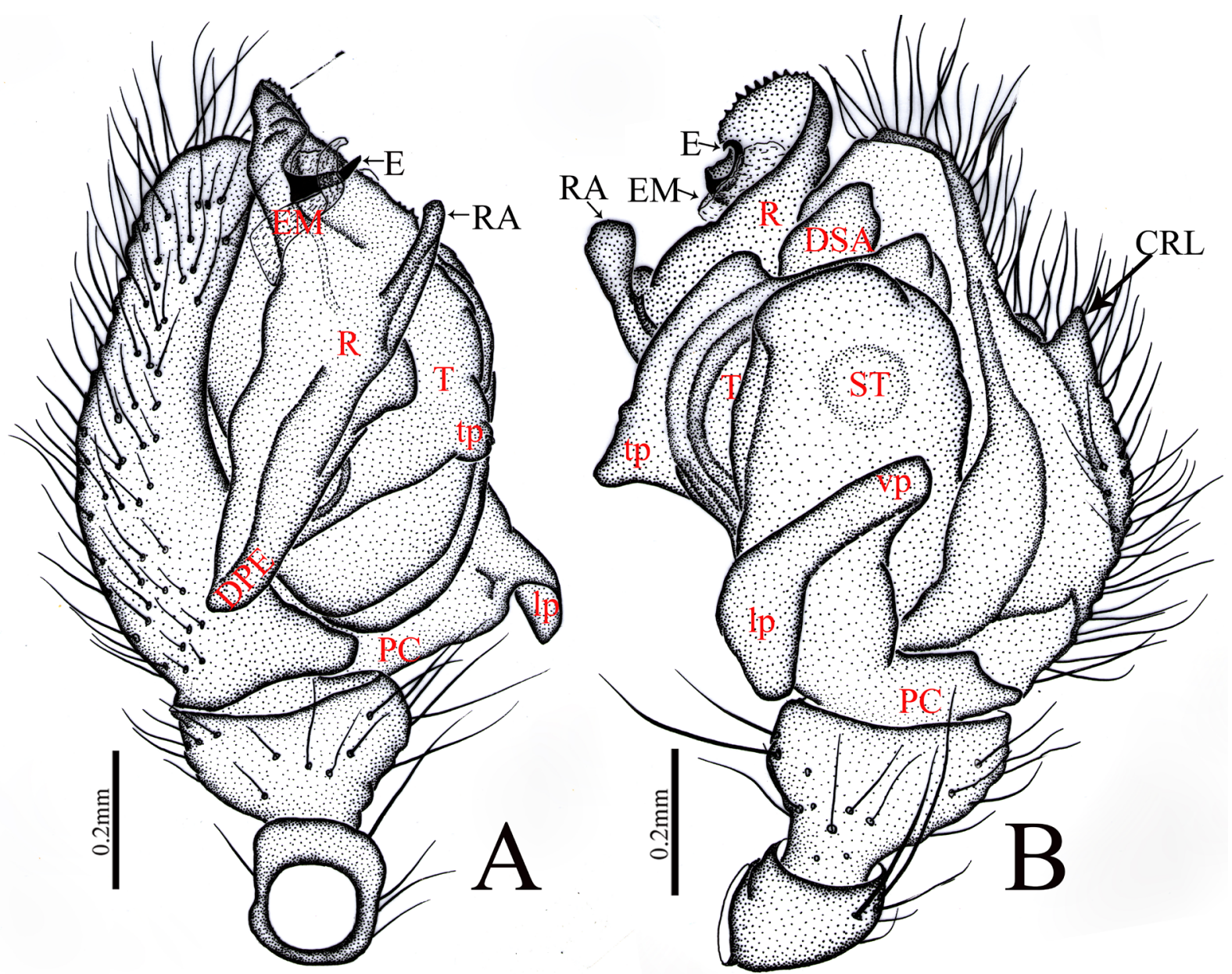

Fig. 26. Acroterius latus gen. et sp. nov., Ô, holotype (HNU-CGY123), palp. A. Prolateral view. B. Retrolateral view. 

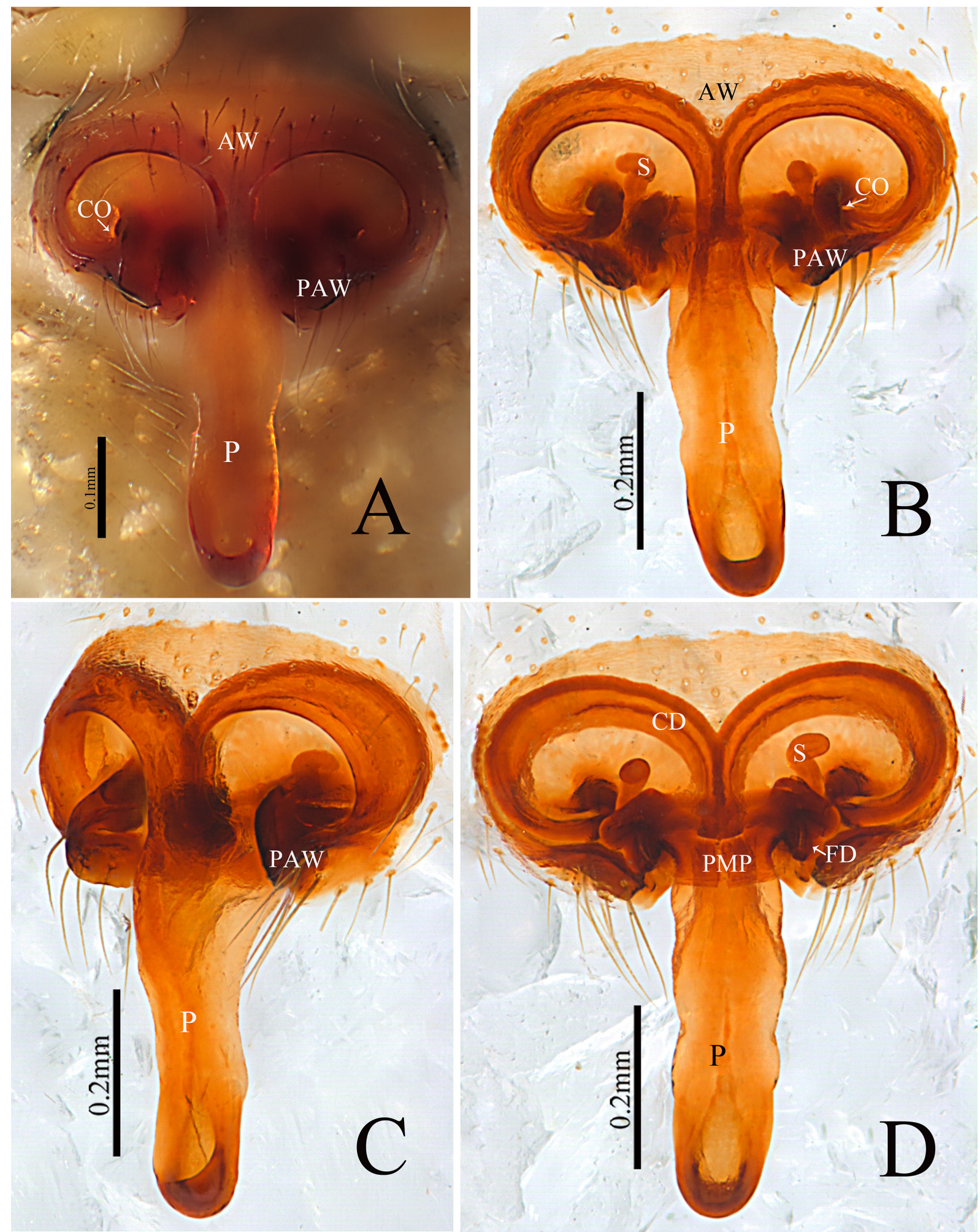

Fig. 27. Acroterius latus gen. et sp. nov., +, paratype (HNU-CGY123). A-B. Epigyne, ventral view. C. Epigyne, lateral view. D. Vulva, dorsal view. 

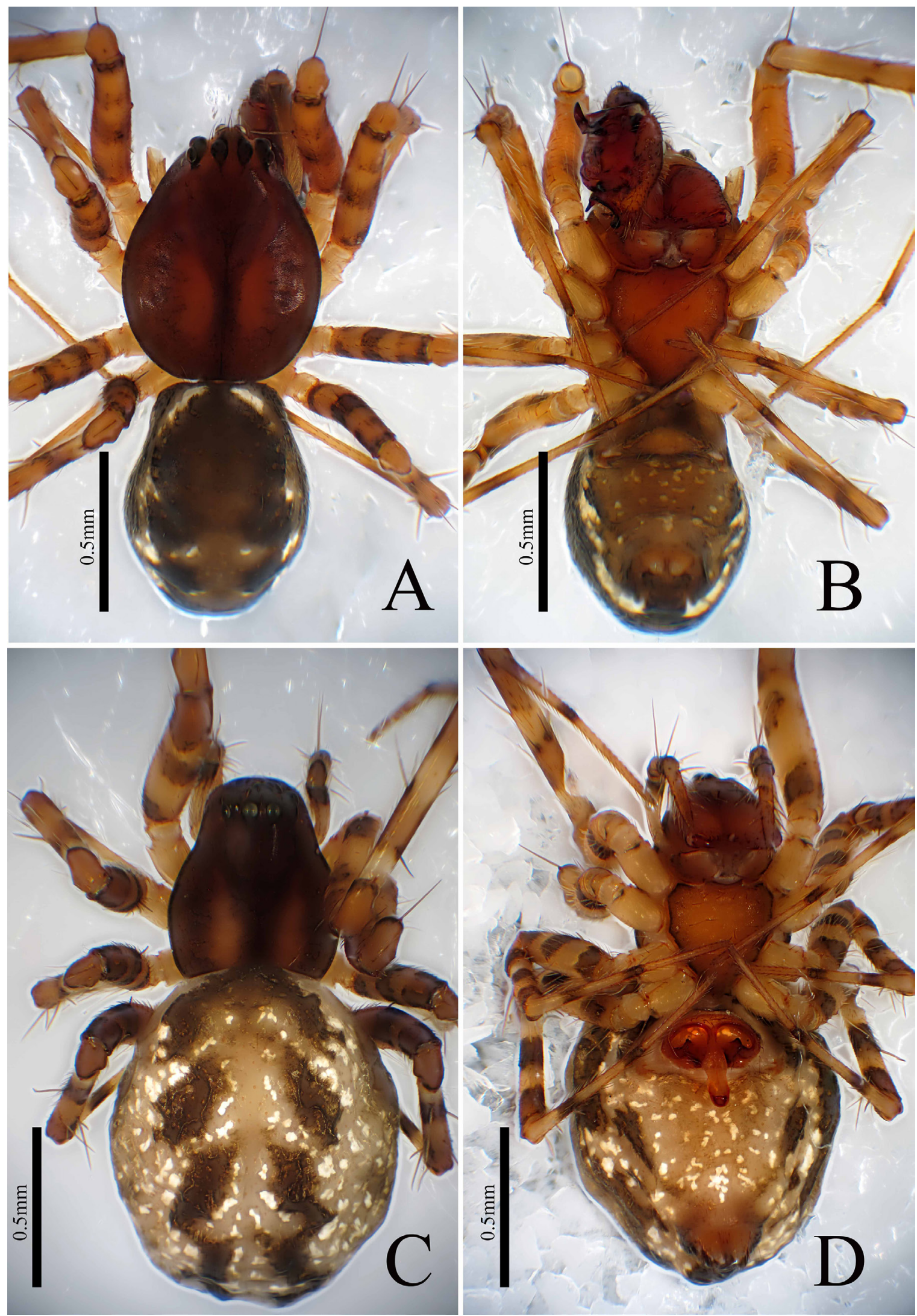

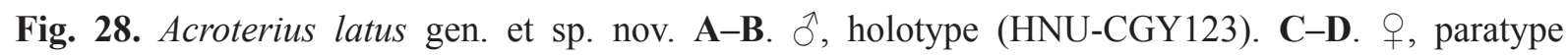
(HNU-CGY123). A, C. Habitus, dorsal view. B, D. Habitus, ventral view. 


\section{Acroterius longidentatus gen. et sp. nov. urn:1sid:zoobank.org:act:F372CAB7-6C3F-403D-809D-4FDA72FB7B10}

Figs 29, 39

\section{Differential diagnosis}

The new species resembles Acroterius brevis gen. et sp. nov. (Fig. 8A-D) in having a similar short parmula as in $A$. camur gen. et sp. nov. and A. longidentatus gen. et sp. nov. and the C-shaped distal part of spermathecae in epigyne (Fig. 29A-D); it can be distinguished by: the posterior projection of the anterior wall of the epigynal plate $\mathrm{C}$-shaped in ventral view, somewhat triangular in lateral view and retained below the lateral margin of anterior wall of the epigyne in the new species (Fig. 29A-C), but beak-shaped in ventral view, protruding above the lateral margin in A. brevis gen. et sp. nov. (Fig. 8A-C). The spermathecae are situated near the median septum (Fig. 29D), but situated away from the median septum in A. brevis gen. et sp. nov. (Fig. 8D). Parmula finger-shaped (Fig. 29A-C), but tongue-shaped in A. brevis gen. et sp. nov. (Fig. 8A-C), anteriorly rectangular and posteriorly spoon-shaped in A. inversus gen. et sp. nov. (Fig. 22A-C) and somewhat triangular in A. camur gen. et sp. nov. (Fig. 13A-C).

\section{Etymology}

The species name comes from the Latin adjective 'longus' + 'dentatus', meaning 'long' + 'teeth' and referring to the long and highly sclerotized teeth on the retrolateral margin of the chelicerae in the female.

\section{Type material}

\section{Holotype}

CHINA - $q$; Yunnan Province, Longling County, Longjiang Township, Xiaoheishan Nature Reserve; $24.82888^{\circ} \mathrm{N}, 98.76001^{\circ} \mathrm{E}$; alt. $2020 \mathrm{~m}$; broadleaf forest; 28 May 2005; Charles Griswold and D.H. Kavanaugh leg.; HNU-2002-033A.

\section{Paratypes $(5 \circ \rightarrow)$}

CHINA - Yunnan Province • 4 q $ᄋ$; same collection data as for holotype; HNU-2002-033A • 1 ; Longling County, Longjiang Township, Xiaoheishan Nature Reserve; $24.83671^{\circ} \mathrm{N}, 98.76185^{\circ} \mathrm{E}$; alt. 2067 m; hand collecting; 28 May 2005; Heng-mei Yan and Guo Ke-ji leg.; HNU-GKJ029.

\section{Description}

Female (holotype)

MeAsurements And Colour. Total length: 3.73 . Carapace 1.27 long, 1.18 wide, cephalic region slightly elevated, yellowish brown, with distinct pattern starting from the base of posterior median eyes; fovea, cervical and radial grooves distinct; clypeus 0.27 high (Fig. 29E-F); abdomen 2.47 long, 1.81 wide. Eye sizes and interdistances: AME 0.07, ALE 0.11, PME 0.08, PLE 0.08, AME-AME 0.05, PME-PME 0.08, AME-ALE 0.11, PME-PLE 0.09, ALE-ALE 0.51, PLE-PLE 0.55, ALE-PLE contiguous.

Chelicerae. With six promarginal and six retromarginal teeth; reteromarginal teeth long and highly sclerotized.

LeNGTH OF LEGS. I $=6.13(1.67,1.95,1.55,0.96), \mathrm{II}=5.50(1.45,1.75,1.51,0.79), \mathrm{III}=3.50(0.98,1.07$, $0.91,0.54), \mathrm{IV}=5.02(1.23,1.61,1.36,0.82)$. Leg formula I-II-IV-III.

EpIGYNe. Parmula 0.36 long, 0.23 wide at base (Fig. 29B); the posterior projection of anterior wall of epigynal plate circular in ventral view; somewhat triangular and retained below lateral wall of epigynal plate in lateral view (Fig. 29A-C). Spermathecae slender, distal part C-shaped and situated near median septum (Fig. 29D). 

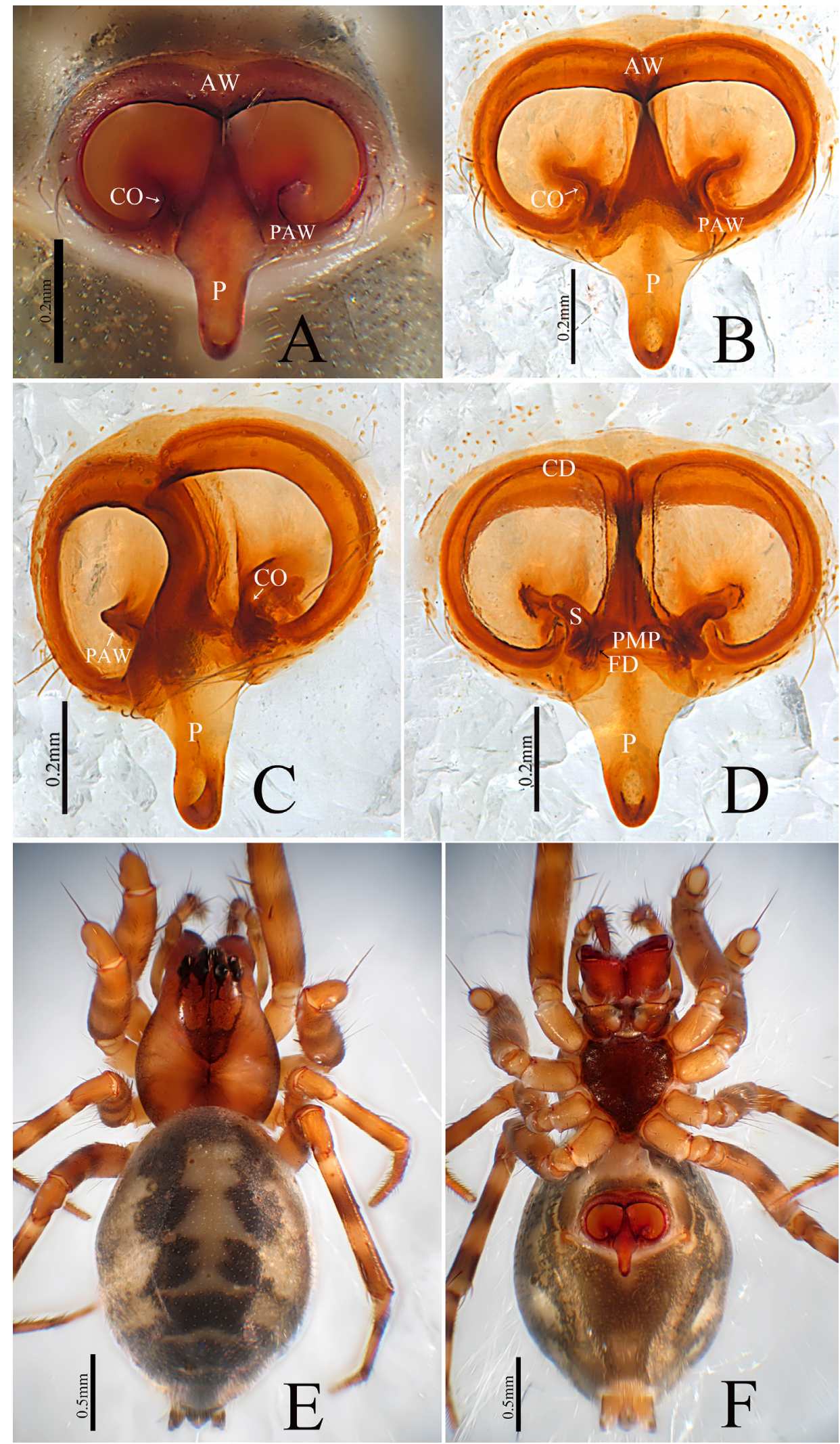

Fig. 29. Acroterius longidentatus gen. et sp. nov., + , holotype (HNU-2002-033A). A-B. Epigyne, ventral view. C. Epigyne, lateral view. D. Vulva, dorsal view. E. Habitus, dorsal view. F. Habitus, ventral view. 


\author{
Male \\ Unknown.

\section{Distribution} \\ China (Yunnan Province, Fig. 39).
}

\author{
Acroterius longimultus gen. et sp. nov. \\ urn:1sid:zoobank.org:act:A79B8C4E-1540-4031-A067-7F12809D5B95
}

Figs 30-33, 39

\title{
Differential diagnosis
}

The new species resembles Acroterius latus gen. et sp. nov. (Figs 24A-D, 26A-B) in having the distal arm of the paracymbium with two projections in the male palp and the long parmula with a median incision in the epigyne (Figs 31A-B, 32A-D); it can be distinguished by: the distal suprategular apophysis long, sword-shaped, protruding beyond the apex of cymbium in retrolateral view (Figs 30B, 31B), but short, retained below the apex of cymbium in A. latus gen. et sp. nov. (Figs 24B, 26B). The dorsal projection of the embolic plate almost touches the base of the cymbium in prolateral view (Figs 30A, 31 A), but doesn't touch the base of the cymbium in A. latus gen. et sp. nov. (Figs 24A, 26A). The lateral projection of the distal arm of the paracymbium is tongue-shaped, away from the posterior margin of the tibia (Figs 30B, $\mathrm{D}, 31 \mathrm{~B}$ ), but finger-shaped, almost touching the posterior margin of the tibia in A. latus gen. et sp. nov. (Figs 24B, D, 26B). The posterior projection of the anterior wall of the epigynal plate is longer than wide, protruding beyond the epigastric furrow (Fig. 32A-B), but wider than long, not protruding beyond the epigastric furrow in A. latus gen. et sp. nov. (Fig. 27A-B). The distal part of spermathecae inverted L-shaped (Fig. 32D), but hook-shaped in A. latus gen. et sp. nov. (Fig. 27D).

\section{Etymology}

The species name comes from the Latin adjective 'longus' + 'multus', meaning 'long' + 'much' and referring to the very long distal suprategular apophysis in the male palp.

\section{Type material}

\section{Holotype}

CHINA - O’; Yunnan Province, Fugong County, Lumadeng Township, Yaping Village, Shibali; 27.16515 N, 98.77975 E; alt. 2527 m; 1 May 2004; Guang-xu leg.; HNU-20040501-1.

Paratypes $(1 \hat{\jmath}, 1$ $)$

CHINA $・ 1$ §, 1 \%; same collection data as for holotype; HNU-20040501-2.

\section{Description}

\section{Male (holotype)}

Measurements. Total length: 3.18. Carapace 1.53 long, 1.23 wide, cephalic region slightly elevated, brown; fovea, cervical and radial grooves distinct; clypeus 0.21 high (Fig. 33A-B); abdomen 1.65 long, 1.19 wide (Fig. 33A-B). Eye sizes and interdistances: AME 0.07, ALE 0.08, PME 0.07, PLE 0.09, AME-AME 0.04, PME-PME 0.07, AME-ALE 0.08, PME-PLE 0.08, ALE-ALE 0.44, PLE-PLE 0.51.

Chelicerae. With five promarginal and five retromarginal teeth; teeth of reteromargin almost equal in length.

LENGTH OF LEGS. I $=5.56(1.39,1.61,1.63,0.93), \mathrm{II}=4.80(1.32,1.43,1.28,0.77), \mathrm{III}=3.87(1.21,1.08$, $1.04,0.54), \mathrm{IV}=5.45(1.39,1.73,1.61,0.72)$. Leg formula I-IV-II-III. 


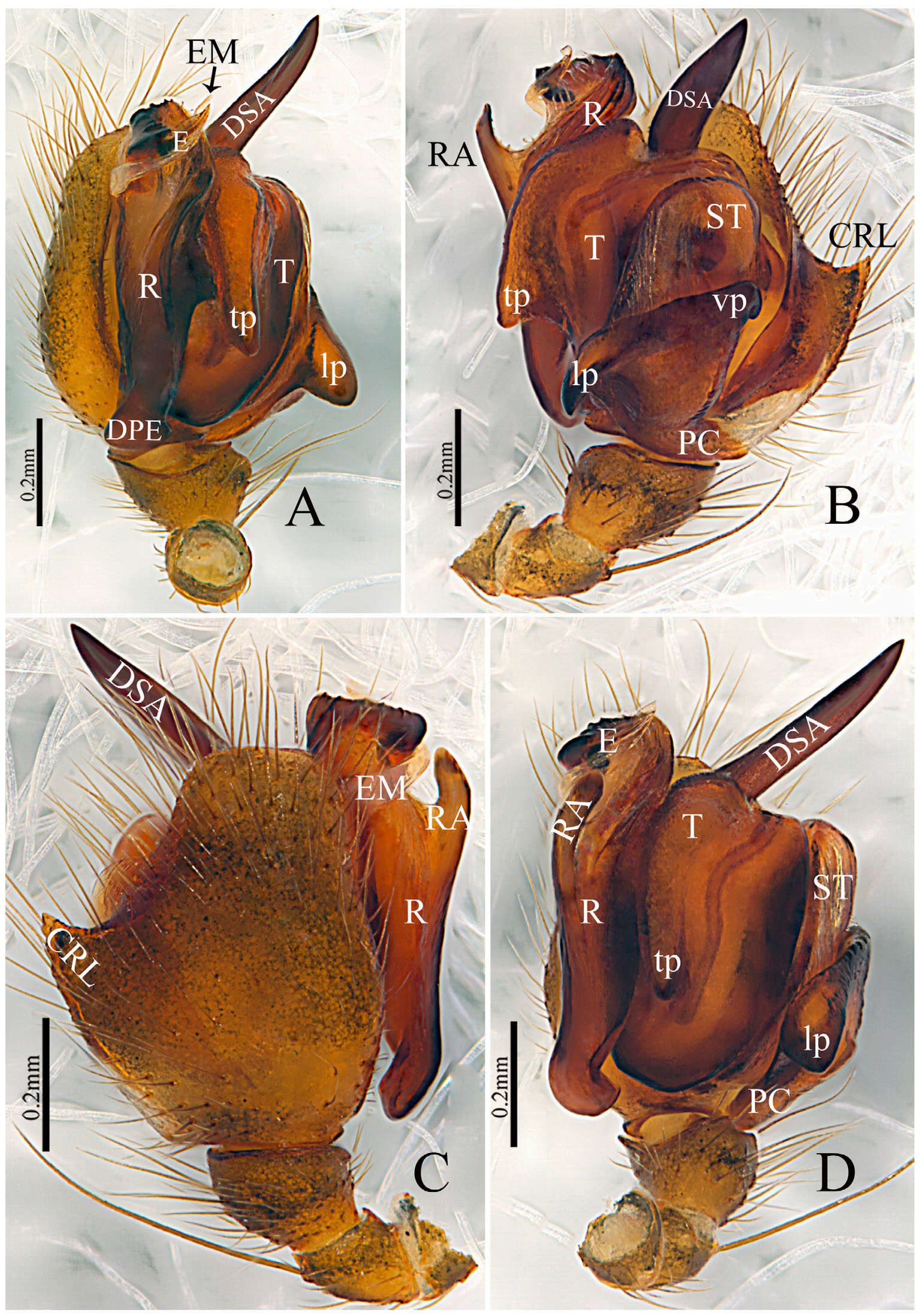

Fig. 30. Acroterius longimultus gen. et sp. nov., §ิ, holotype (HNU-20040501-1), palp. A. Prolateral view. B. Retrolateral view. C. Dorsal view. D. Ventral view. 
PaLP (Figs 30A-D, 31A-B). Paracymbium highly sclerotized, distal arm with three projections; lateral projection large, curved, beak-shaped with pointed end in retrolateral view; median projection tongueshaped, wider than long; ventral projection sclerotized, tip curved with blunt end, strongly overlapping subtegulum (Figs 30B, 31B); distal suprategular apophysis much longer than that of all other species in this genus, sword-shaped, protruding above cymbium (Figs 30A-D, 31A-B). Radical apophysis longer than wide, finger-shaped with broad blunt end, pointing towards distal margin of radix in ventral view (Figs 30A-D, 31A-B). Dorsal projection of embolic plate with broad, round end, curved and almost touching base of cymbium in prolateral view (Figs 30A, 31A). Embolus stout, short with pointed end (Figs 30A-C, 31A-B).

\section{Female (HNU-20040501-02)}

MEASUREMENTS. Total length: 2.67. Carapace 1.13 long, 0.91 wide, cephalic region slightly elevated, yellowish brown, with longitudinal band starting from base of posterior lateral eyes extending to middorsal; fovea, cervical and radial grooves distinct; Clypeus 0.24 high (Fig. 33D-E); abdomen 1.54 long, 1.16 wide. Eye sizes and interdistances: AME 0.06, ALE 0.08, PME 0.06, PLE 0.07, AME-AME 0.04, PME-PME 0.05, AME-ALE 0.06, PME-PLE 0.09, ALE-ALE 0.39, PLE-PLE 0.42, ALE-PLE contiguous.

Chelicerae. With five promarginal and five retromarginal teeth.
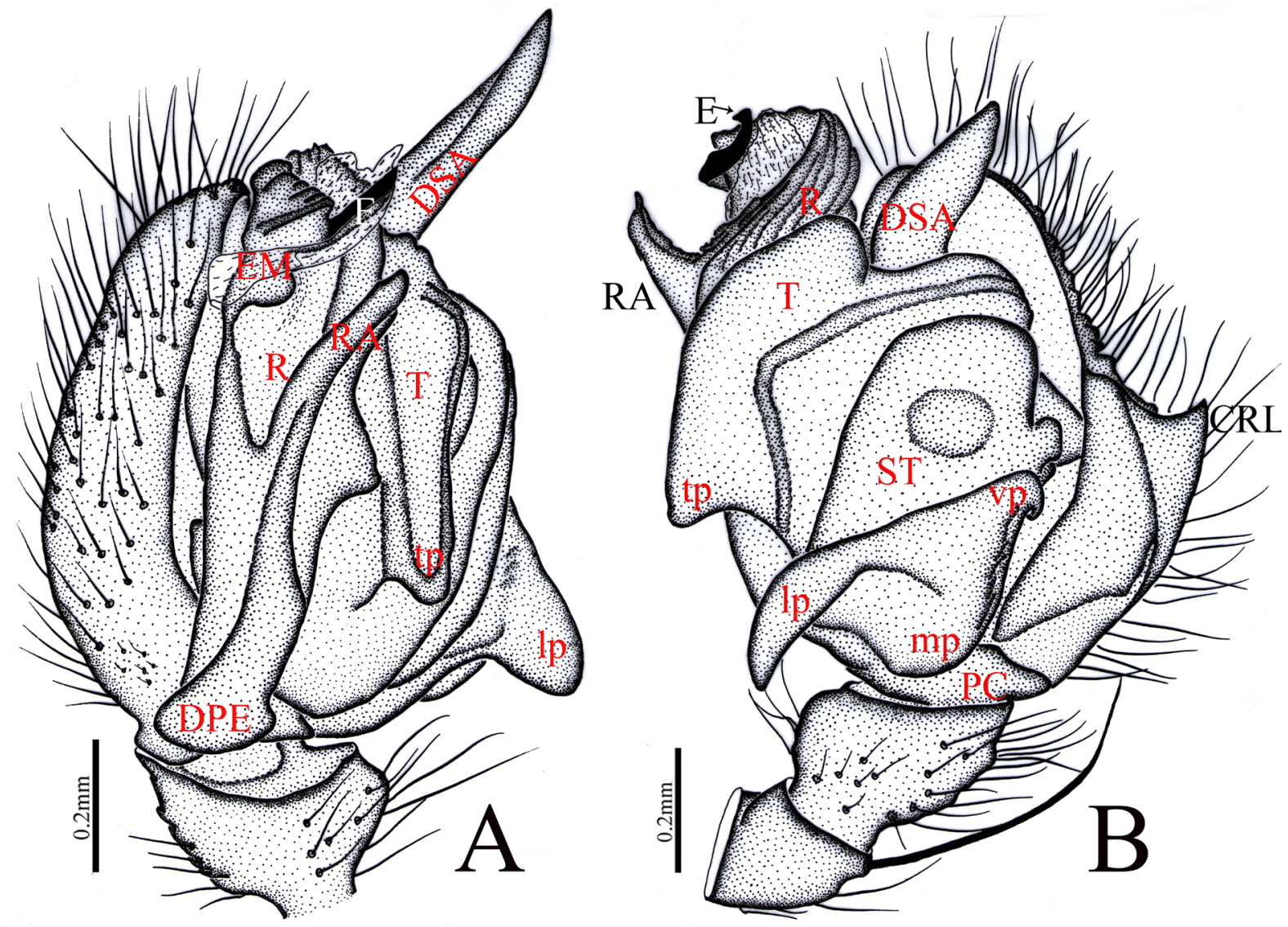

Fig. 31. Acroterius longimultus gen. et sp. nov., §, holotype (HNU-20040501-1), palp. A. Prolateral view. B. Retrolateral view. 

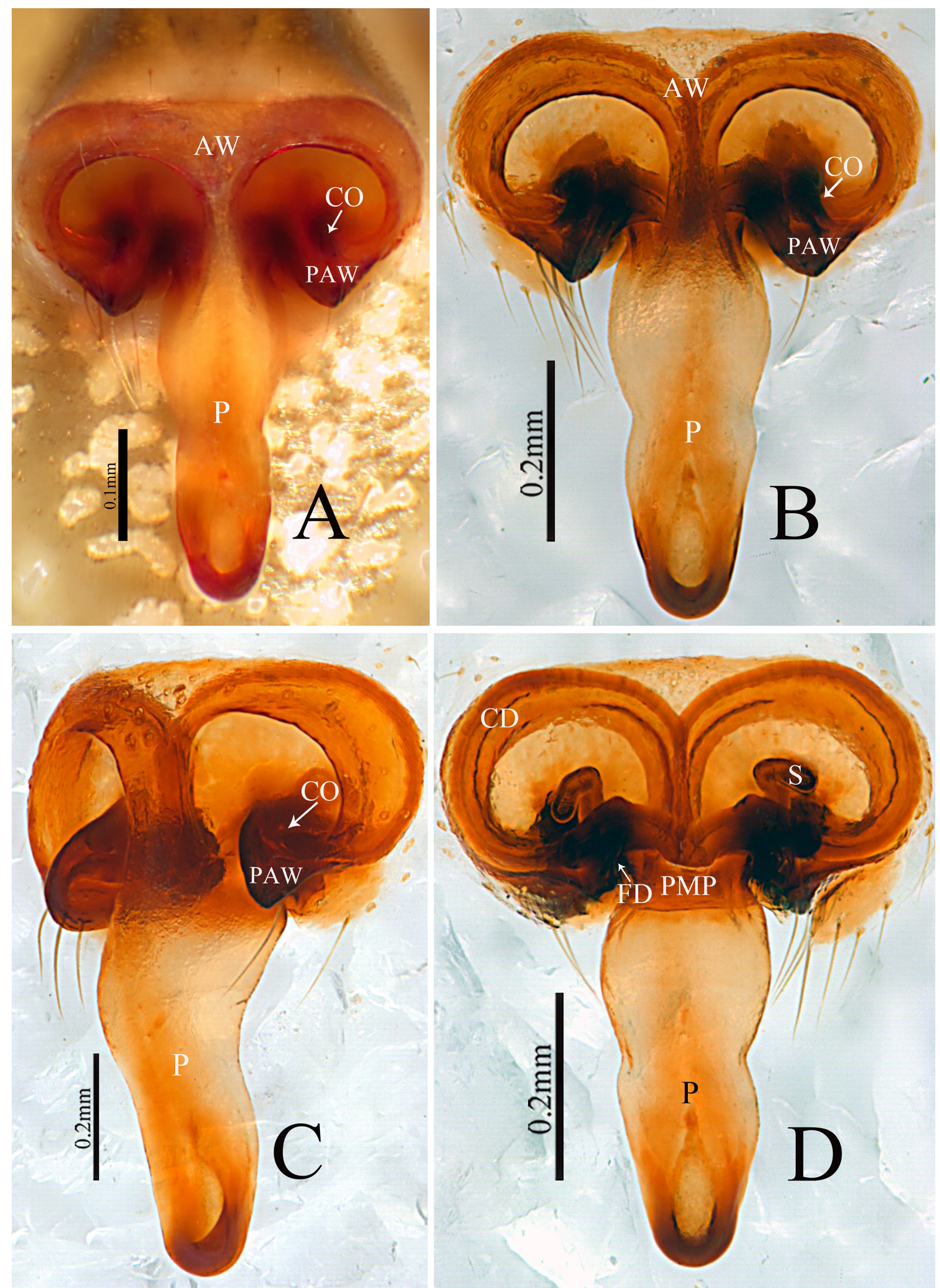

Fig. 32. Acroterius longimultus gen. et sp. nov., +, paratype (HNU-20040501-02). A-B. Epigyne, ventral view. C. Epigyne, lateral view. D. Vulva, dorsal view. 
LENGTH OF LeGs. I $=3.88(1.19,1.31,0.86,0.52), \mathrm{II}=3.74(1.15,1.08,0.88,0.63), \mathrm{III}=2.62(0.8,0.84$, $0.61,0.37), \mathrm{IV}=3.37(0.93,1.03,0.86,0.55)$. Leg formula I-II-IV-III.

EpigYNe. Lateral margin of parmula wave-like on each side (Fig. 32A-C); parmula 0.45 long, 0.15 wide at base (Fig. 32A-C); posterior projection of anterior wall of epigynal plate longer than wide and protruding beyond epigastric furrow (Fig. 32A-B). Spermathecae slender, inverted L-shaped (Fig. 32D).

\section{Distribution}

China (Yunnan Province, Fig. 39).

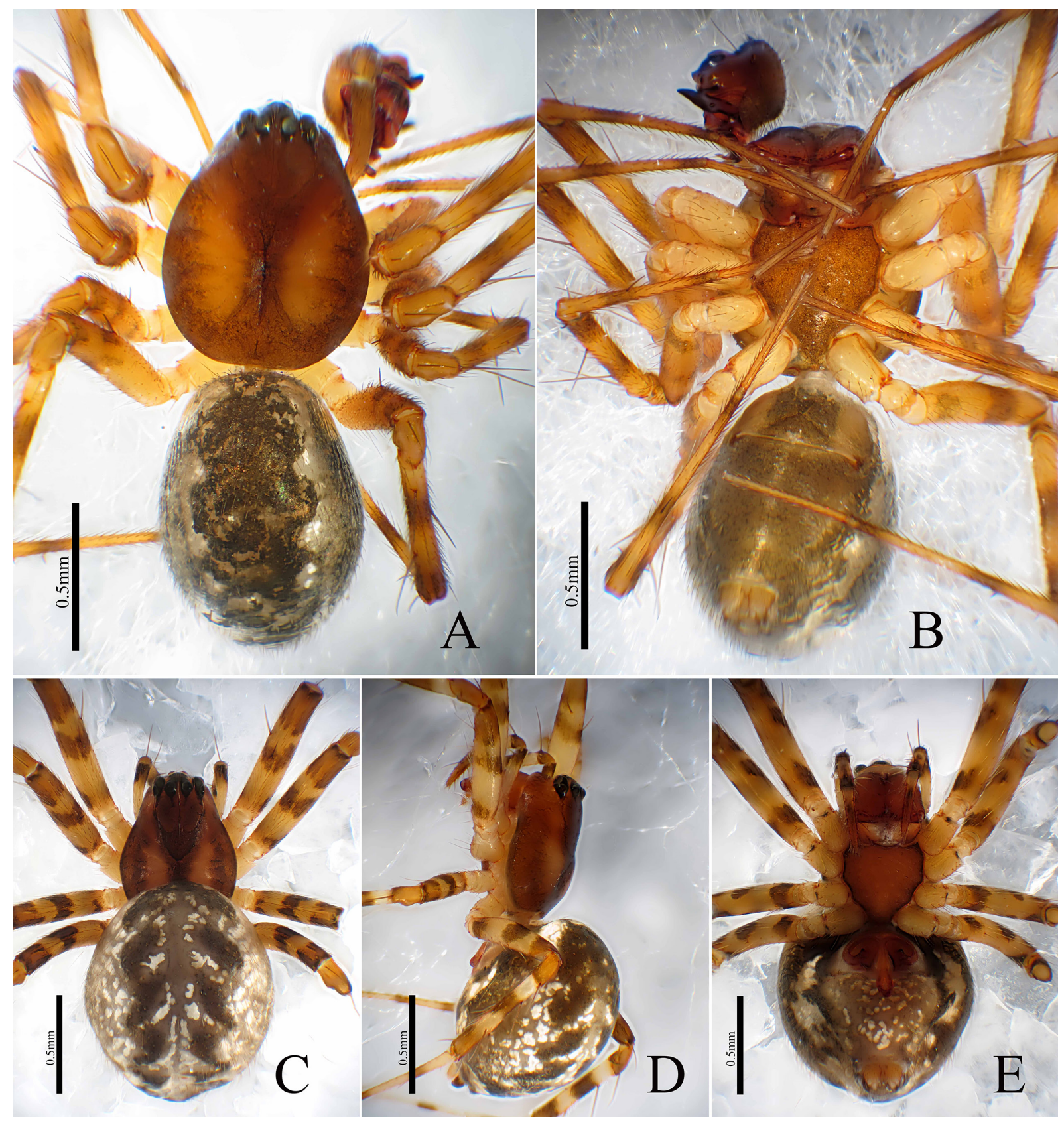

Fig. 33. Acroterius longimultus gen. et sp. nov. A-B. đ, holotype (HNU-20040501-1). C-E. \&, paratype (HNU-20040501-02). A, C. Habitus, dorsal view. B, E. Habitus, ventral view. D. Habitus, lateral view. 
Acroterius longiprojectus gen. et sp. nov. urn:1sid:zoobank.org:act:5E2F3303-07EE-4A75-9FE8-62EC8372BB8C

Figs 34, 39

\title{
Differential diagnosis
}

The new species resembles Acroterius hamatus gen. et sp. nov. (Fig. 19A-D) in having long parmula in epigyne (Fig. 34B-E); it can be distinguished by: the posterior projection of the anterior wall of the epigynal plate longer than wide (Figs 34B-C), but wider than long in A. hamatus gen. et sp. nov. (Figs 19A-B, 21A-B). In ventral view, the distal part of parmula is not expanded (Fig. 34B-C), but distinctly expanded in A. hamatus gen. et sp. nov. (Fig. 19A-B). The spermathecae are not curved (Fig. 34E), but the tip is curved, pointing towards the posterior margin of the epigyne in A. hamatus gen. et sp. nov. (Figs 19D, 21D).

\section{Etymology}

The species name comes from the Latin adjective 'longus' + 'projectus' meaning 'long' + 'projection', referring to the long posterior projection of the anterior wall of the epigynal plate in the epigyne.

\section{Type material}

\section{Holotype}

CHINA • P; Yunnan Province, Fugong County, Lumadeng Township, Yakou of old Shibali; $27.06430^{\circ} \mathrm{N}$, 98.75123ํ E; alt. 3270 m; 13 Aug. 2005; Guo Tang leg.; HNU-Tang-05-06.

\section{Description}

Female (holotype)

Measurements And Colour. Total length: 3.15 . Carapace 1.33 long, 0.97 wide, cephalic region slightly elevated, brown, dorsally with distinct pattern starting from base of posterior eyes extending to proximal part of cephalothorax; fovea, cervical and radial grooves distinct; clypeus 0.26 high (Fig. 34A); Abdomen 1.83 long, 1.48 wide (Fig. 34A). Eye sizes and interdistances: AME 0.06, ALE 0.08, PME 0.06, PLE 0.07 AME-AME 0.04, PME-PME 0.06, AME-ALE 0.07, PME-PLE 0.09, ALE-ALE 0.42, PLE-PLE 0.45 , ALE-PLE contiguous.

Chelicerae. With six promarginal and six retromarginal teeth; reteromarginal teeth long and highly sclerotized.

LENGTH OF LEGS. I $=4.66(1.14,1.35,1.2,0.77), \mathrm{II}=3.78(0.95,1.2,1.11,0.52), \mathrm{III}=2.45(0.84,1.02$, $1.11,0.49), \mathrm{IV}=4.16(1.17,1.23,1.11,0.65)$. Leg formula I-IV-II-III.

Epigyne. Parmula 0.49 long, 0.27 wide at base (Fig. 34B-C); posterior projection of anterior wall of epigynal plate longer than wide on either side (Fig. 34B-C). Spermathecae slender, long, almost touches lateral wall of epigyne on each side (Fig. 34E).

\author{
Male \\ Unknown.

\section{Distribution} \\ China (Yunnan Province, Fig. 39).
}



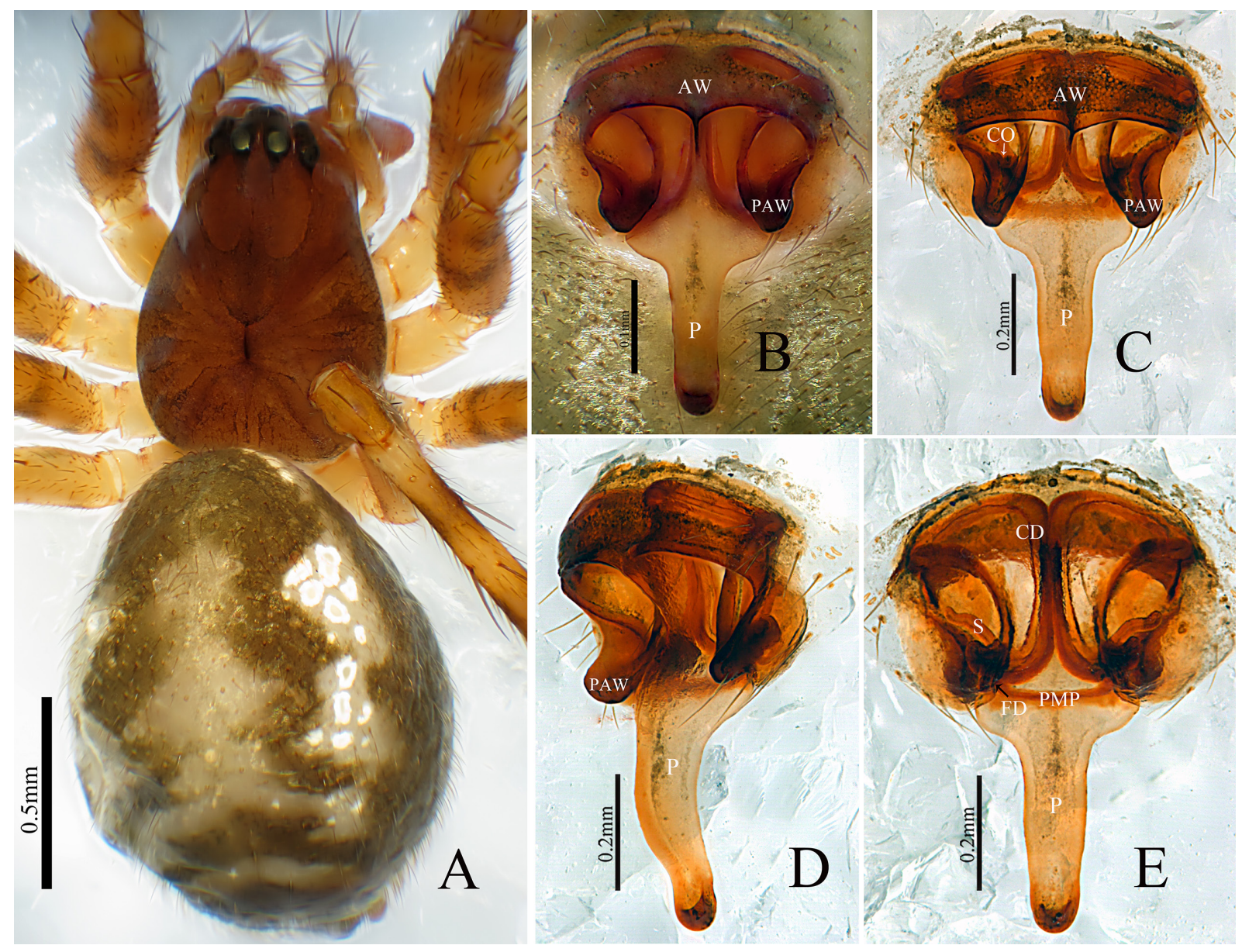

Fig. 34. Acroterius longiprojectus gen. et sp. nov.,, , holotype (HNU-Tang-05-06). A. Habitus, dorsal view. B-C. Epigyne, ventral view. D. Epigyne, lateral view. E. Vulva, dorsal view.

Acroterius ovatus gen. et sp. nov. urn:1sid:zoobank.org:act:33E64A5A-FD47-48ED-B075-B721D9EDE3A3

Figs 35-36, 39

\section{Differential diagnosis}

The new species resembles Acroterius longidentatus gen. et sp. nov. (Fig. 29A-C) in having a similar circular posterior projection of the anterior wall of the epigynal plate in the epigyne (Fig. 36A-C); it can be distinguished by the anterior wall of the oval epigynal plate margin (Fig. 36A-B), but semicircular in A. longidentatus gen. et sp. nov. (Fig. 29A-B). In dorsal view, parmula with wave-like margin and almost four times as long as median septum (Fig. 36D), but tongue-shaped and as long as median septum in A. longidentatus gen. et sp. nov. (Fig. 29D). The spermathecae are hook-shaped (Fig. 36D), but C-shaped in A. longidentatus gen. et sp. nov. (Fig. 29D).

\section{Etymology}

The species name comes from the Latin adjective 'ovatus', meaning 'oval' and referring to the oval anterior wall of the epigynal plate margin of the epigyne. 


\section{Type material}

\section{Holotype}

CHINA • + ; Yunnan Province, Tengchong County, Mingguang Township, Zizhi Village; $25.79808^{\circ} \mathrm{N}$, 98.62406 ${ }^{\circ}$ E; alt. 2756 m; 19 May 2006; Chang-min Yin, Jia-fang Hu and Ming-wei Yang leg.; HNU-YHY06.

\section{Description}

Female (holotype)

Measurements and COlour. Total length: 3. Carapace 1.01 long, 0.96 wide, cephalic region slightly elevated, dark brown, dorsally with distinct pattern starting from base of posterior eyes extending to proximal part of cephalothorax; fovea, cervical and radial grooves distinct; clypeus 0.23 high (Fig. 35AB); abdomen 1.99 long, 1.49 wide (Fig. 35A-B). Eye sizes and interdistances: AME 0.06, ALE 0.09, PME 0.08, PLE 0.07, AME-AME 0.04, PME-PME 0.06, AME-ALE 0.09, PME-PLE 0.07, ALE-ALE 0.45, PLE-PLE 0.48, ALE-PLE 0.02.

Chelicerae. With five promarginal and six retromarginal teeth; reteromarginal teeth long and highly sclerotized, protruding beyond fangs.
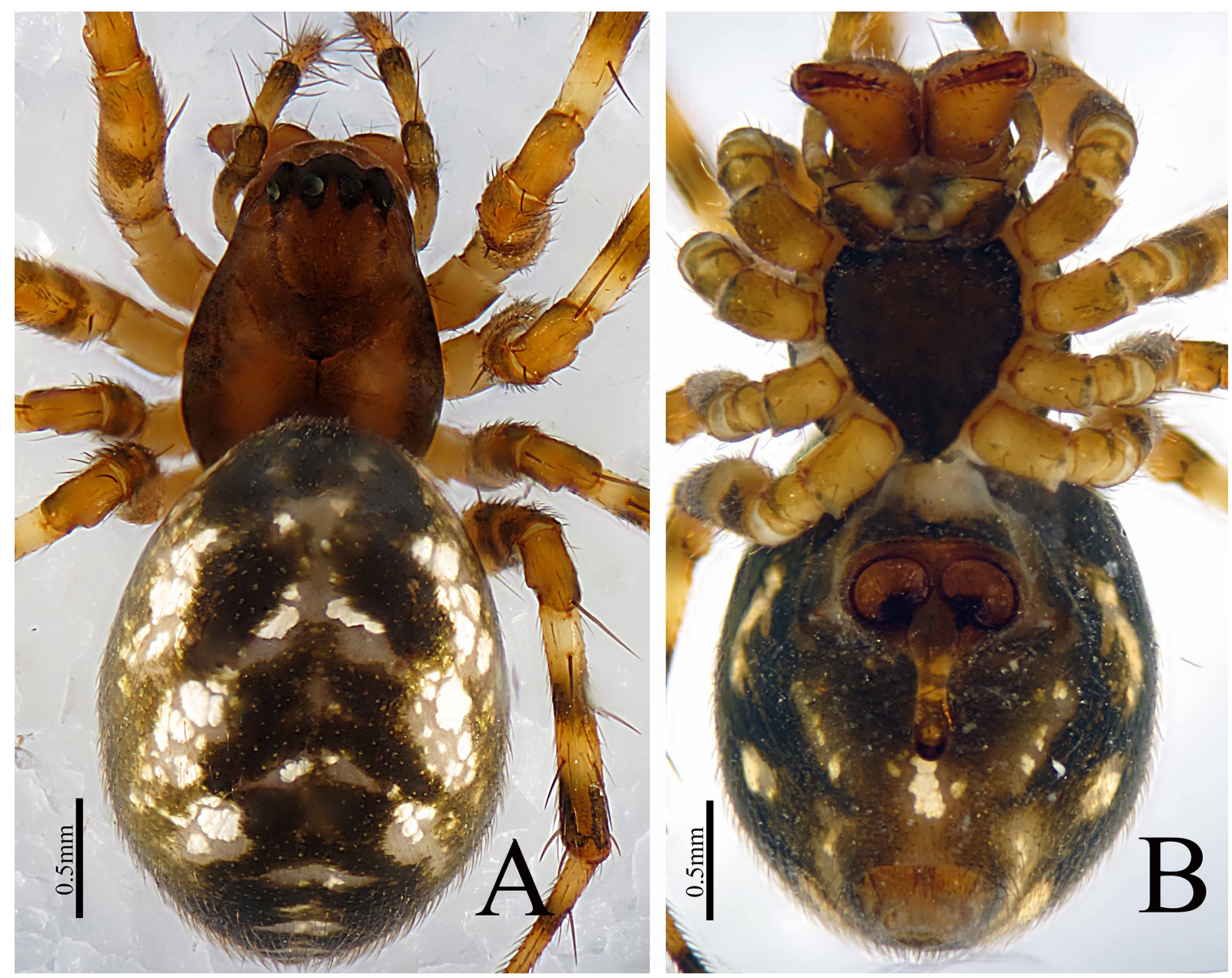

Fig. 35. Acroterius ovatus gen. et sp. nov., $\uparrow$, holotype (HNU-YHY06). A. Habitus, dorsal view. B. Habitus, ventral view. 


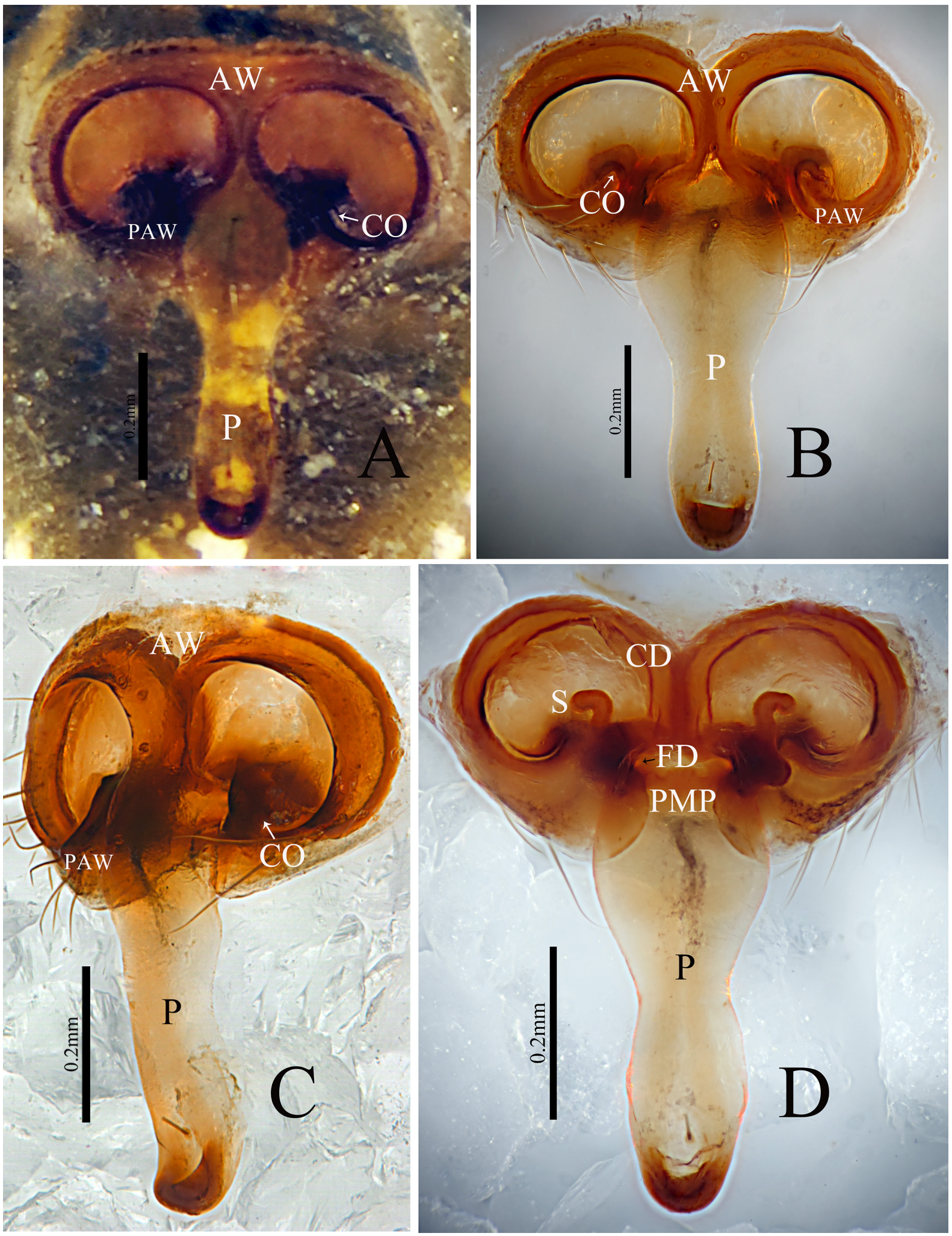

Fig. 36. Acroterius ovatus gen. et sp. nov.,, , holotype (HNU-YHY06). A-B. Epigyne, ventral view. C. Epigyne, lateral view. D. Vulva, dorsal view. 
LeNGTH OF LeGs. I $=4.34(1.19,1.35,1.07,0.73), \mathrm{II}=4.03(1.14,1.28,0.89,0.72), \mathrm{III}=2.76(0.94,0.72$, $0.65,0.45), \mathrm{IV}=3.56(1.15,1.08,0.74,0.59)$. Leg formula I-II-IV-III.

EpIGYNe. Lateral margin of parmula wave-like (Fig. 36A-B); parmula 0.57 long, 0.18 wide at base (Fig. 35A-B); anterior wall of epigynal plate margin oval (Fig. 36A-B); posterior projection of anterior wall of epigynal plate somewhat circular on each side (Fig. 36A-B). Spermathecae slender, hook-shaped pointing towards lateral margin of epigyne (Fig. 36D).

\section{Male}

Unknown.

\section{Distribution}

China (Yunnan Province, Fig. 39).

Acroterius parvus gen. et sp. nov. urn:lsid:zoobank.org:act:959DFFDC-D4A9-41C5-B099-B7AA5047F2D8

Figs 37,39

\section{Differential diagnosis}

The new species resembles Acroterius brevis gen. et sp. nov. in having similar hook-shaped spermathecae; it can be distinguished by the triangular parmula, shorter than the median septum in the new species (Fig. 37A, D), but tongue-shaped and longer than the median septum in A. brevis gen. et sp. nov. (Fig. 8A-B, D).

\section{Etymology}

The species name comes from the Latin adjective 'parvus', meaning 'small', referring to the small parmula of the epigyne.

\section{Type material}

\section{Holotype}

CHINA - ; Y Yunnan Province, Gongshan County, Bingzhongluo Township, Chukuai; $27.97928^{\circ}$ N, 98.47389 E; alt. 3725 m; 19 Aug. 2006; Peng Hu leg.; HNU-Hu060819.

Paratype $(1$ $)$

CHINA • 1 + ; same collection data as for holotype; HNU-Hu060819.

\section{Description}

Female (holotype)

MEAsurements AND COLOUR. Total length: 4.15. Carapace 1.60 long, 2.90 wide, cephalic region slightly elevated, brown, dorsally with dark brown longitudinal band starting from base of posterior eyes extending to proximal part of cephalothorax; fovea, cervical and radial grooves distinct; clypeus 0.41 high (Fig. 37E-F); abdomen 2.55 long, 2.35 wide. Eye sizes and interdistances: AME 0.13, ALE 0.1, PME 0.09, PLE 0.12, AME-AME 0.06, PME-PME 0.08, AME-ALE 0.07, PME-PLE 0.12, ALE-ALE 0.51, PLE-PLE 0.62, ALE-PLE 0.03.

Chelicerae. With six promarginal and six retromarginal teeth; reteromarginal teeth long and highly sclerotized. 

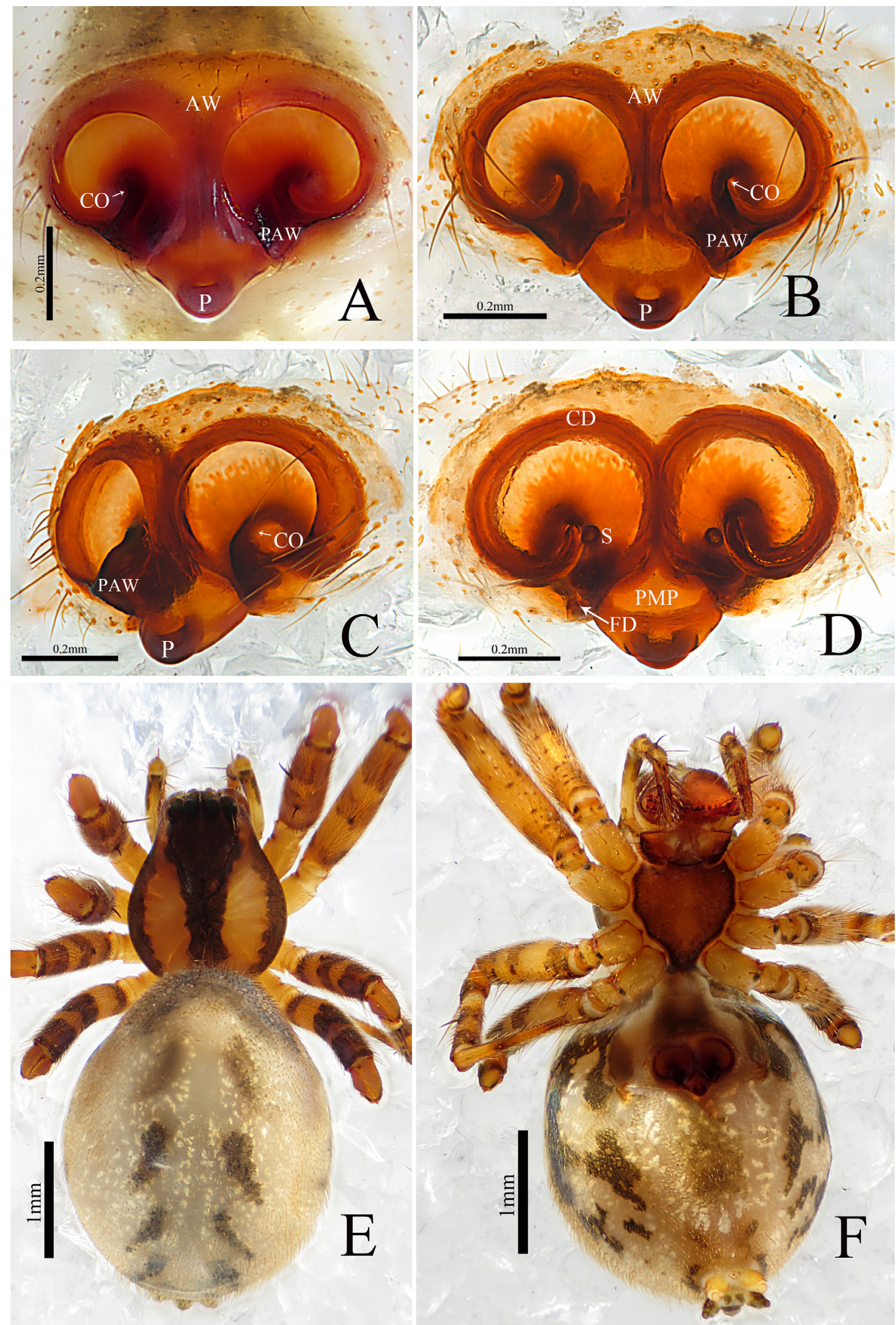

Fig. 37. Acroterius parvus gen. et sp. nov., + , holotype (HNU-Hu060819). A-B. Epigyne, ventral view. C. Epigyne, lateral view. D. Vulva, dorsal view. E. Habitus, dorsal view. F. Habitus, ventral view. 
LENGTH OF LeGs. I $=7.25(1.97,2.24,1.83,1.21), \mathrm{II}=6.48(1.87,1.89,1.69,1.03), \mathrm{III}=4.63(1.52,1.34$, $1.16,0.61), \mathrm{IV}=6.02(1.75,1.84,1.54,0.89)$. Leg formula I-II-IV-III.

EPIGYNe. Posterior median plate extends posteriorly with small triangular parmula, wider than long (Fig. 37A-B); parmula 0.26 long, 0.26 wide at base (Fig. 37A-B); posterior projection of anterior wall of epigynal plate circular in ventral view, somewhat triangular in lateral view on each side (Fig. 37A-B). Spermathecae slender, distal part slightly curved and pointing towards fertilization ducts (Fig. 37D).

\section{Male \\ Unknown.}

\section{Distribution}

China (Yunnan Province, Fig. 39).

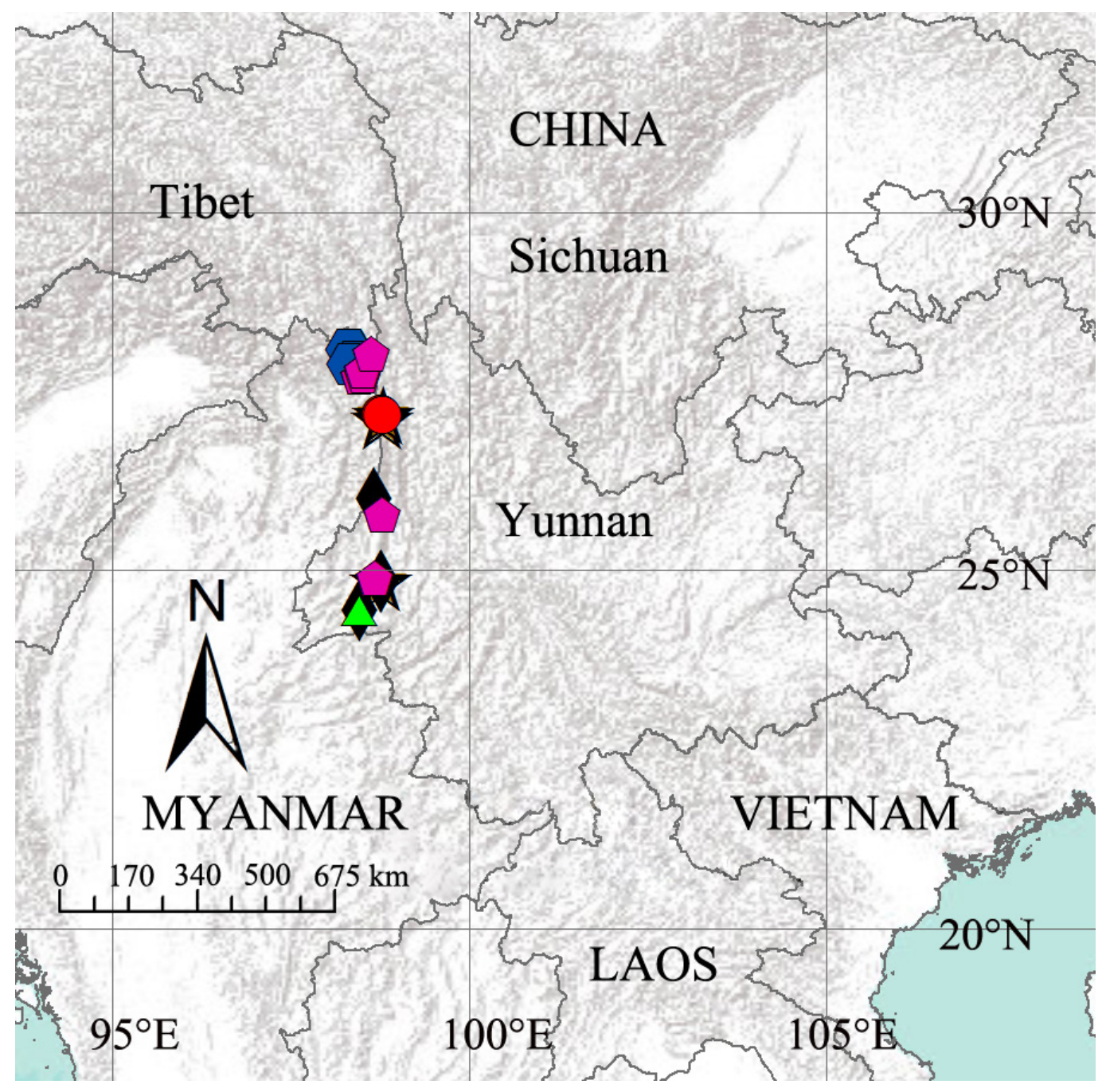

Fig. 38. Map indicating the type localities of Acroterius absentus sp. nov. ( $\triangle$ ), A. brevis sp. nov. A. camur sp. nov. (○), A. hamatus sp. nov. (○), A. inversus sp. nov. (\) and A. latus sp. nov. ( $\star$ ). 


\section{Discussion}

Based on illustrations and morphological characters provided by Merrett (1963), Acroterius gen. nov. can be identified as a member of the subfamily Linyphiinae given that: a claw is present on the female palp; the male palp lacks a tibial apophysis; the maxillae are long and parallel; and all tibiae have two dorsal spines. The discovery of this new genus is not surprising, considering that there is limited taxonomic research on Linyphiidae from Yunnan. Zhao \& Li (2014, 2017), Irfan \& Peng (2018, 2019a, 2019b) and Irfan et al. (2019) already identified 39 species and 10 genera; with the addition of Acroterius gen. nov., the number of linyphiid genera endemic to Yunnan is now eleven. Acroterius gen. nov. appears to be closely related to Zhezhoulinyphia as many species in both genera have been recorded from the same localities and they share similarities in the embolic division and spermathecae shape. Considering their morphological similarities and overlapping distribution, it is possible that these genera are sister taxa and their ancestor originated in the mountains of southwestern Yunnan. However, more taxonomic and phylogenetic studies need to be done on Linyphiidae from Yunnan and across the adjacent areas to understand the distribution and origins of these taxa.

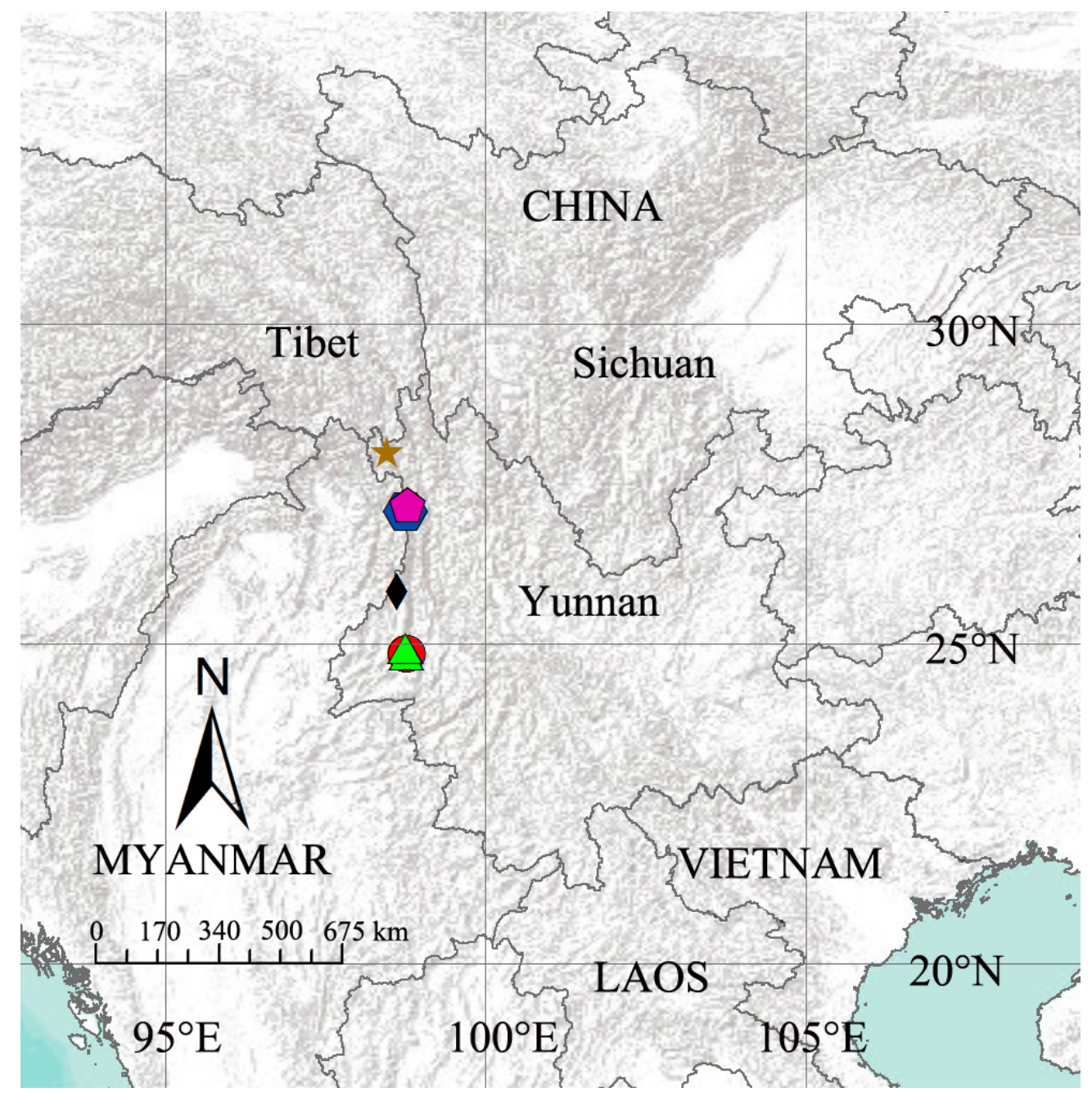

Fig. 39. Map indicating the type localities of Acroterius circinatus sp. nov. ( $\triangle$ ), A. longidentatus sp. nov. (O), A. longimultus sp. nov. (○), A. longiprojectus sp. nov. (○), A. ovatus sp. nov. (() and A. parvus sp. nov. ( $(\star)$. 


\section{Acknowledgements}

The authors are particularly grateful to all the people who directly contributed to this study by collecting the studied material, in particular: Chang-Min Yin late, Jia-Fang Hu, Ming-Wei Yang, Heng-Mei Yang, Guo Tang, Guang-Xu Peng, Charles Griswold, D.H. Kavanaugh, P.E. Marek, Darrell Ubick, Da-Zhi Dong, Hong-Bin Liang, Ke-Ji Guo and Peng Hu. The authors are also thankful to Dr Andrei V. Tanasevitch for his suggestions and confirmation of embolic division structures and also indebted to the anonymous reviewers for their useful comments and suggestions to improve the manuscript. This research was sponsored by the National Science Foundation of the USA through the "joint biodiversity survey of The Sino-American, California Academy of Sciences (CAS)" in grant "Biotic survey of the Gaoligong Mountain, a biodiversity hotspot in western Yunnan, China" (No. DEB-0103795). It is also partly supported by the National Natural Sciences Foundation of China (NSFC-30970327, 31272271, 31272272, 31301861), National Special Fund on Basic Research of Science and Technology of China (No. 2014FY110100), Hunan Provincial Natural Science Foundation of China (No.11JJ1004/12JJ3028), Program for New Century Excellent Talents in University (NCET-12-0717), China Postdoctoral Science Foundation (No. 20100471221/201104506), the program of Hunan Provincial Science and Technology Plans (No. 2010RS4006), and by the Hunan Provincial Program for Development of Key Disciplines in Ecology (grant number No. 0731).

\section{References}

Irfan M. \& Peng X.J. 2018. Three new species of Linyphiidae (Arachnida: Araneae) from Yunnan, China. Oriental Insects 52 (3): 229-244. https://doi.org/10.1080/00305316.2017.1398115

Irfan M. \& Peng X.J. 2019a. Herbiphantes Tanasevitch, 1992 and Labullinyphia van Helsdingen, 1985 (Araneae, Linyphiidae), two newly recorded spider genera from the Gaoligong Mountains in China with the description of two new species. Zootaxa 4638 (4): 547-561.

https://doi.org/10.11646/zootaxa.4638.4.5

Irfan M. \& Peng X.J. 2019b. The genus Parbatthorax Tanasevitch, 2019 (Araneae, Linyphiidae) new to China, with a new species from the Gaoligong Mountains. European Journal of Taxonomy 555: 1-19. https://doi.org/10.5852/ejt.2019.555

Irfan M., Zhou G.C. \& Peng X.J. 2019. Zhezhoulinyphia gen. nov. (Araneae, Linyphiidae) from Yunnan, China. ZooKeys 862: 43-60. https://doi.org/10.3897/zookeys.862.31406

Ivie W. 1969. North American spiders of the genus Bathyphantes (Araneae, Linyphiidae). American Museum Novitates 2364: 1-70.

Li S.Q. \& Lin Y.C. 2016. Species Catalogue of China. Volume 2: Animals Invertebrates (I) Arachnida: Araneae. Science Press, Beijing.

Merrett P. 1963. The palpus of male spiders of the family Linyphiidae. Proceedings of the Zoological Society of London 140 (3): 347-467. https://doi.org/10.1111/j.1469-7998.1963.tb01867.x

Millidge A.F. 1988. The spiders of New Zealand: Part VI. Family Linyphiidae. Otago Museum Bulletin 6: 35-67.

Paquin P. \& Dupérré N. 2003. Guide d'identification des araignées de Québec. Fabreries, Supplement 11: $1-251$.

Tao Y., Li S.Q. \& Zhu C.D. 1995. Linyphiid spiders of Changbai Mountains, China (Araneae: Linyphiidae: Linyphiinae). Beiträge zur Araneologie 4: 241-288.

Van Helsdingen P.J. 1972. An account of money spiders from down under (Araneida, Linyphiidae). Zoologische Mededelingen 47: 369-390. 
Zhao Q.Y. \& Li S.Q. 2014. A survey of linyphiid spiders from Xishuangbanna, Yunnan Province, China (Araneae, Linyphiidae). ZooKeys 460: 1-181. https://doi.org/10.3897/zookeys.460.7799

Zhao Q.Y. \& Li S.Q. 2017. Callosa gen. n., a new troglobitic genus from southwest China (Araneae, Linyphiidae). ZooKeys 703: 109-128. https://doi.org/10.3897/zookeys.703.13641

Manuscript received: 6 May 2020

Manuscript accepted: 9 February 2021

Published on: 1 April 2021

Topic editor: Rudy Jocqué

Desk editor: Kristiaan Hoedemakers

Printed versions of all papers are also deposited in the libraries of the institutes that are members of the EJT consortium: Muséum national d'histoire naturelle, Paris, France; Meise Botanic Garden, Belgium; Royal Museum for Central Africa, Tervuren, Belgium; Royal Belgian Institute of Natural Sciences, Brussels, Belgium; Natural History Museum of Denmark, Copenhagen, Denmark; Naturalis Biodiversity Center, Leiden, the Netherlands; Museo Nacional de Ciencias Naturales-CSIC, Madrid, Spain; Real Jardín Botánico de Madrid CSIC, Spain; Zoological Research Museum Alexander Koenig, Bonn, Germany; National Museum, Prague, Czech Republic. 